\title{
Synthesis of Enantiopure Oxygen- and Nitrogen-Containing Heterocycles by Diastereoselective Ring-Closing Metathesis Reaction in Perhydro-1,3-benzoxazine Derivatives
}

\author{
Agustín Gutiérrez-Loriente, ${ }^{a}$ José M. Martín-Álvarez, ${ }^{\mathrm{b}}$ Elena Prieto, ${ }^{\mathrm{a}}$ Celia Andrés ${ }^{\mathrm{a} *}$ \\ and Javier Nieto ${ }^{\mathrm{a} *}$ \\ a Instituto CINQUIMA and Departamento de Química Orgánica, Facultad de Ciencias, Universidad de Valladolid, Paseo \\ de Belén, 7, 47011 Valladolid, Spain \\ E-mails: javiernr@qo.uva.es \\ b Instituto CINQUIMA and Departamento de Química Inorgánica, Facultad de Ciencias, Universidad de Valladolid, \\ Paseo de Belén, 7, 47011 Valladolid, Spain
}

\begin{abstract}
Diastereoselective ring-closing metathesis reactions on chiral trienic perhydro-1,3-benzoxazines derived from (-)-8-aminomenthol featuring two diastereotopic olefin chains is described. The diastereochemical outcome of the cyclization appeared to be dependent on the length and position of the olefin chains in perhydro-1,3-benzoxazine, the degree of substitution of the double bonds and the ruthenium catalyst used. After separation of the diastereomers, and removal of the chiral auxiliary, enantiopure oxygenand nitrogen-containing heterocycles were obtained.
\end{abstract}

Keywords: Asymmetric synthesis; metathesis; oxygen heterocycles; nitrogen heterocycles; chiral auxiliaries

\section{Introduction}

Partially unsaturated oxygen and nitrogen-containing heterocycles are common subunits displayed in a broad array of interesting and useful biologically active molecules as well as natural products. ${ }^{[1]}$ Among a large number of synthetic approaches, the ring-closing metathesis (RCM) reaction has emerged as one of the most efficient ways to prepare differentsized heterocycles. ${ }^{[2-4]} \mathrm{A}$ wide range of enantioenriched heterocycles can be accessed by asymmetric ring-closing metathesis (ARCM), asymmetric ring-opening/cross-metathesis (AROCM) or asymmetric ring rearrangements (ARR) starting from racemic or pro-chiral compounds in the presence of chiral well-defined molybdenum- and ruthenium-based metathesis catalysts. ${ }^{[5]}$ Despite progress in chiral catalyst design, however, a significant challenge remains, ARCM of unhindered trienes has so far been unsuccessful, resulting in extremely low enantioselectivities. ${ }^{[6]}$ In these cases, the diastereoselective ring-closing metathesis reaction $(\mathrm{DSRCM})^{[7]}$ in substrates in which an existing chiral centre controls the direction of cyclization could compete advantageously over an enantioselective RCM reaction using chiral alkylidene complexes. Under certain reaction conditions the DSRCM cyclization may be reversible and the observed diastereomer ratios reflect a thermodynamic preference. $^{[8]}$ If one of the diastereomers is energetically much more favoured good diastereoselectivities can be achieved not only under kinetic control, but also under thermodynamic control. ${ }^{[9]}$ On the other hand, the diastereomers could be easily separated by chromatography and the desired product can be obtained optically pure. ${ }^{[10]}$

Over the last few years, we have shown that chiral perhydro-1,3-benzoxazines derived from (-)-8aminomenthol constitute useful chiral auxiliaries in different diastereoselective cycloaddition and cyclization processes. ${ }^{[11]}$ We now report on the synthesis of enantiopure cyclic ethers and amines by DSRCM reaction on trienic perhydro-1,3benzoxazines with two diastereotopic olefinic groups derived from (-)-8-aminomenthol acting as chiral template. ${ }^{[12]}$ Scheme 1 summarizes our intentions. In general, performing DSRCM metathesis reactions with high diastereoselectivity requires that the olefins at the prochiral centre do not react with each other and also that the primary attack of the catalyst occurs at the double bond of the other olefin. ${ }^{\text {[7a] }}$ 


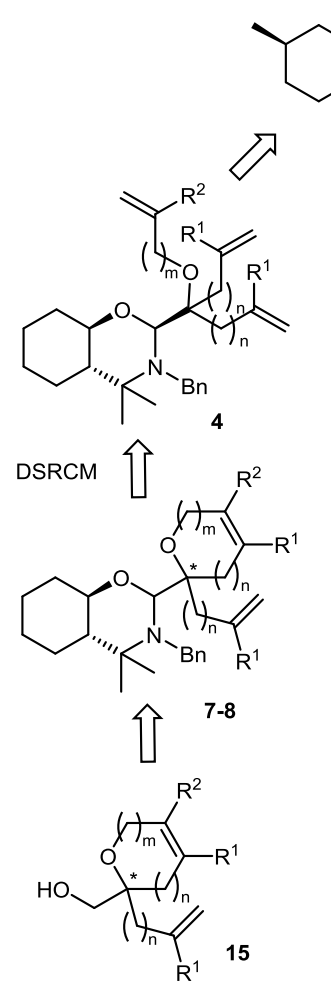<smiles>C=CC(C)(C)NC(C)(C)C1CCCC1O</smiles>
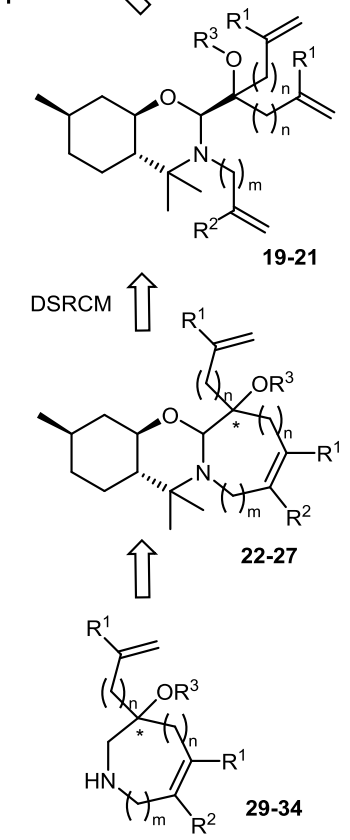

Scheme 1. Retrosynthetic approach to chiral oxygen and nitrogen-containing heterocycles.

\section{Results and Discussion}

\section{Synthesis of chiral cyclic ethers}

The starting trienic perhydro-1,3-benzoxazines $\mathbf{4 a - j}$ were prepared as single diastereomers in good chemical yields from (-)-8-(benzylamino)menthol $\mathbf{1},{ }^{[13]}$ in three steps, as summarized in Scheme 2. Condensation of the amino alcohol $\mathbf{1}$ with ethyl glyoxylate in toluene at reflux furnished a mixture of equatorial 2a and axial $\mathbf{2 b}$ esters in 85:15 ratio which could be separated by silica column chromatography. However, $\mathbf{2 b}$ is quite configurationally unstable and quickly reverts to $\mathbf{2 a} .^{[14]}$ Treatment of either $\mathbf{2 a}$ or the epimeric mixture of $\mathbf{2 a}$ and $\mathbf{2 b}$ with vinylic or allylic Grignard reagents gave the same equatorial carbinols 3a-d that were converted into the final perhydrobenzoxazines $\mathbf{4 a - g}$ by allylation the hydroxyl group.

Initial experiments were conducted on perhydro1,3-benzoxazine 4a with monosubstituted olefinic appendages. Reactions were carried out in $\mathrm{CH}_{2} \mathrm{Cl}_{2}$ at room temperature. In the presence of a $4 \mathrm{~mol} \%$ of the first-generation Grubbs' catalyst 5, 4a was converted in a mixture of dihydrofurans 7a and $\mathbf{8 a}$ in good yield and moderate diastereoselectivity (entry 1 in Table 1). Employment of the Grubbs' second generation catalyst 6 resulted in a reverse stereoinduction, ${ }^{[15]}$ but the stereoselectivity remained moderate (entry 2 in Table 1).

Although it is conceivable that the difference in the product ratios of the cyclization in the presence of 5

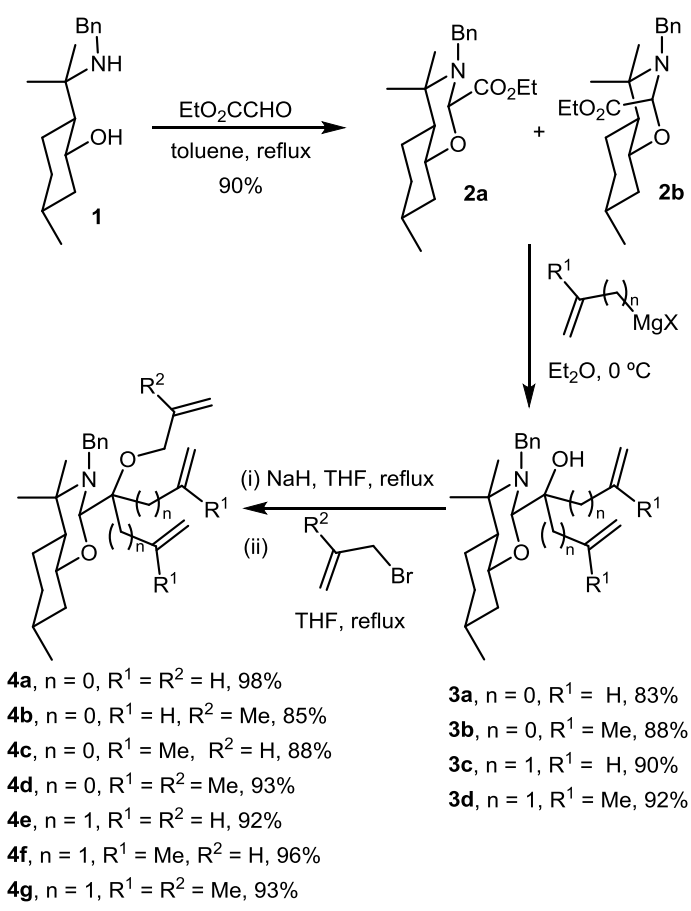

Scheme 2. Synthesis of perhydrobenzoxazines $4 \mathbf{a}-\mathbf{g}$.

or 6 reflect a kinetic or thermodynamic preference, it is quite unlikely that the reaction of $\mathbf{4 a}$ with the most active ruthenium(II)-N-heterocyclic carbene complex 6 will take under thermodynamic rather than kinetic control in the tested reaction conditions for several reasons: (i) the product ratios do not change with time; for example, the reaction of $\mathbf{4 a}$ in the presence of $\mathbf{6}$ for $30 \mathrm{~h}$ led to $7 \mathbf{a}(35 \%)$ and $\mathbf{8 a}(65 \%)$, almost the same ratio of diastereomers as after $55 \mathrm{~h}$ (entry 3); (ii) running the reaction in $\mathrm{CH}_{2} \mathrm{Cl}_{2}$ at $40{ }^{\circ} \mathrm{C}$ or in toluene at $110{ }^{\circ} \mathrm{C}$ for $30 \mathrm{~h}$ resulted in partial degradation of the material without any significant change in the diastereoselectivity (entries 4 and 5); (iii) once isolated, diastereomers $7 \mathbf{a}$ and $8 \mathbf{a}$ do not interconvert when subjected to the reaction conditions even in ethylene atmosphere. We believe that this catalyst-specificity is attributed to the different spatial arrangements of the respective ligands during the cyclization.

In a similar way triene $4 \mathbf{e}$ with monosubstituted olefinic appendages provided dihydropyran $\mathbf{7 e}$ as major isomer in the presence of $\mathbf{5}$ and dihydropyran $8 \mathbf{e}$ in the presence of $\mathbf{6}$ (entries 11 and 12). When the first-generation Grubbs' catalyst $\mathbf{5}$ was used, the cyclopentene resulting from RCM reaction of the diastereotopic olefin chains was also isolated in a $20 \%$ yield. This compound was also formed initially when the second-generation Grubbs' catalyst $\mathbf{6}$ was used but over time it was transformed in a mixture of $7 \mathbf{e}$ and $\mathbf{8 e}$ by a rearrangement involving a diastereoselective ring-opening metathesis reaction. As with $\mathbf{4 a}$, a detailed monitoring of the reaction of $4 \mathbf{e}$ in the presence of $\mathbf{6}$ showed that proportion of diastereoisomers $\mathbf{7 e}$ and $8 \mathrm{e}$ do not change with 
Table 1. DSRCM reaction of trienes 4a-g.

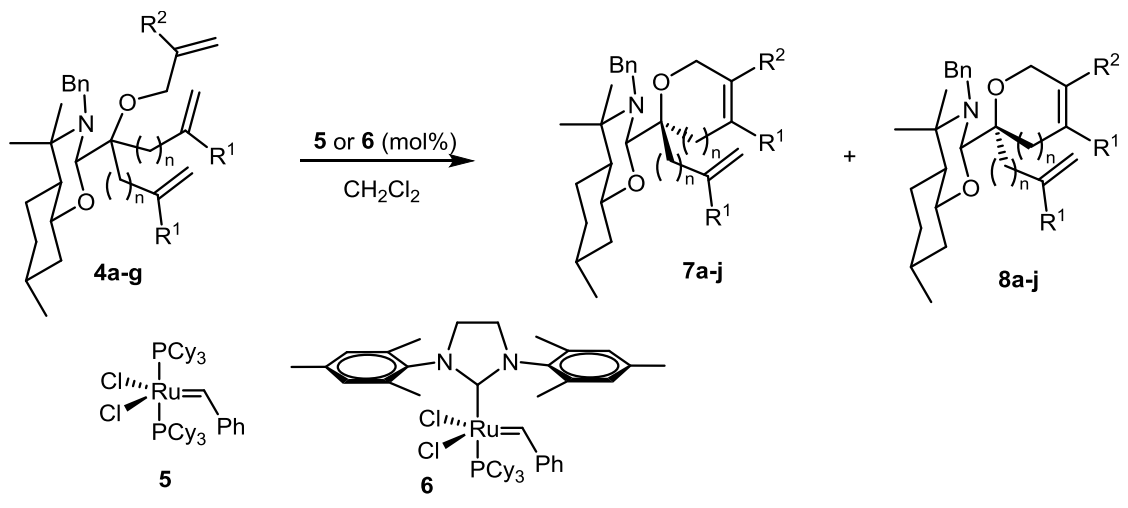

\begin{tabular}{|c|c|c|c|c|c|c|c|c|c|}
\hline Entry & 4 & $\mathrm{n}$ & $\mathrm{R}^{1}$ & $\mathrm{R}^{2}$ & T. $\left({ }^{\circ} \mathrm{C}\right)$ & Catalyst (mol) ${ }^{[\mathrm{a}]}$ & $t(\mathrm{~h})^{[\mathrm{a}]}$ & Yield $(\%)^{[\mathrm{b}]}$ & $d r(\%)^{[\mathrm{c}]}$ \\
\hline 1 & $4 a$ & 0 & $\mathrm{H}$ & $\mathrm{H}$ & 20 & $5(2+2)$ & $15+10$ & 89 & $7 \mathbf{a}(64) / \mathbf{8 a}(36)$ \\
\hline 2 & $4 a$ & 0 & $\mathrm{H}$ & $\mathrm{H}$ & 20 & $6(2+2)$ & $15+15$ & 79 & $\mathbf{7 a}(35) / \mathbf{8 a}(65)$ \\
\hline 3 & $4 a$ & 0 & $\mathrm{H}$ & $\mathrm{H}$ & 20 & $6(2+2+2)$ & $15+15+25$ & 56 & $\mathbf{7 a}(33) / \mathbf{8 a}(67)$ \\
\hline 4 & $4 a$ & 0 & $\mathrm{H}$ & $\mathrm{H}$ & 40 & $6(2+2)$ & $15+15$ & 68 & $\mathbf{7 a}(33) / \mathbf{8 a}(67)$ \\
\hline 5 & $4 \mathbf{a}$ & 0 & $\mathrm{H}$ & $\mathrm{H}$ & $110^{[\mathrm{d}]}$ & $6(2+2)$ & $15+15$ & 55 & $7 \mathbf{a}(32) / \mathbf{8 a}(68)$ \\
\hline 6 & $4 b$ & 0 & $\mathrm{H}$ & $\mathrm{Me}$ & 40 & $5(2+2)$ & $15+10$ & $--_{-[}^{\text {[e] }}$ & --- \\
\hline 7 & $4 b$ & 0 & $\mathrm{H}$ & $\mathrm{Me}$ & 40 & $6(2+2)$ & $15+15$ & $30^{[\mathrm{e}]}$ & $\mathbf{7 b}(47) / \mathbf{8 b}(53)$ \\
\hline 8 & $4 c$ & 0 & $\mathrm{Me}$ & $\mathrm{H}$ & 40 & $5(2+2)$ & $15+15$ & $53^{[e]}$ & $7 \mathbf{c}(>98)^{[\mathrm{f}]}$ \\
\hline 9 & $4 c$ & 0 & $\mathrm{Me}$ & $\mathrm{H}$ & 20 & $6(2)$ & 12 & 92 & $\mathbf{7 c}(>98)^{[\mathrm{f]}}$ \\
\hline 10 & 4d & 0 & $\mathrm{Me}$ & $\mathrm{Me}$ & 40 & $6(2+2+2)$ & $15+15+10$ & 86 & $\mathbf{7 d}(83) / \mathbf{8 d}(17)$ \\
\hline 11 & $4 e$ & 1 & $\mathrm{H}$ & $\mathrm{H}$ & 20 & $5(2)$ & 15 & 75 & $\mathbf{7 e}(77) / \mathbf{8 e}(23)$ \\
\hline 12 & $4 e$ & 1 & $\mathrm{H}$ & $\mathrm{H}$ & 20 & $6(2)$ & 15 & 96 & $7 \mathbf{e}(36) / 8 \mathbf{e}(64)$ \\
\hline 13 & $4 f$ & 1 & $\mathrm{Me}$ & $\mathrm{H}$ & 20 & $5(2)$ & 12 & 94 & $\mathbf{7 f}(78) / \mathbf{8 f}(22)$ \\
\hline 14 & $4 f$ & 1 & $\mathrm{Me}$ & $\mathrm{H}$ & 20 & $6(2)$ & 12 & 93 & $\mathbf{7 f}(36) / \mathbf{8 f}(64)$ \\
\hline 15 & $4 \mathrm{~g}$ & 1 & $\mathrm{Me}$ & $\mathrm{Me}$ & 40 & $\mathbf{6}(2+2+2)$ & $15+15+10$ & 84 & $\mathbf{7 g}(72) / \mathbf{8 g}(28)$ \\
\hline
\end{tabular}

${ }^{\text {[a] }}$ Catalyst was replenished after the time indicated in the Table; see also Experimental Section. ${ }^{[b]}$ Yield refers to pure compounds after column chromatography. ${ }^{[c]}$ Determined by ${ }^{1} \mathrm{H}$ NMR on the reaction mixtures. ${ }^{[d]}$ Toluene was used as solvent. ${ }^{[\mathrm{e}]}$ Different amounts of the starting materials were recovered. ${ }^{[\mathrm{f}]}$ Only one diastereomer was detected by ${ }^{1} \mathrm{H}$ NMR spectra of the reaction mixture.

reaction time. The low diastereoselectivity observed in the cyclization of $\mathbf{4 a}$ and $\mathbf{4 e}$ is presumably due to competing initiation at the various olefinic sites.

The effect of olefin substitution on stereoselectivity was studied in trienes $\mathbf{4 b - \mathbf { d }}$ and $\mathbf{4 f}-\mathbf{g}$ (entries 6-10 and 13-15). Triene 4b with two diastereotopic vinyl chains and a 1,1-disubstituted olefinic chain cyclized with difficulty only in the presence of the most active catalyst $\mathbf{6}$ and with almost no diastereoselectivity (entries 6-7). On the contrary, the reaction of DRCM occurred with greater diastereoselectivity and better chemical yields when the two diastereotopic olefinic chains are 1,1disubstituted at the double bond. Even dihydrofurans and dihydropyrans with a tetrasubstituted double bond can be prepared with good chemical yield in the presence of $\mathbf{6}$ (entries 10 and 15). The best result in terms of diastereoselectivity was obtained in the cyclization of $\mathbf{4 c}$ which gave rise to the formation of a single diastereomer $\mathbf{7 c}$, in the presence of both $\mathbf{5}$ and $\mathbf{6}$ (entries 8 and 9).

The diastereomers formed in each reaction were isolated and purified by flash chromatography and the absolute configuration of the newly created stereocentres in 7c, 8d, $8 \mathbf{e}$ and $\mathbf{8 g}$ was established by $\mathrm{X}$-ray diffraction studies. ${ }^{[16]}$ On the other hand, the absolute configuration of the newly created stereocentre in $\mathbf{7 f}$ was determined by its conversion into the perhydro-1,3-benzoxazine 9 by hydrogenation of the double bonds and hydrogenolysis of the benzyl group (Scheme 3), and X-ray diffraction analysis. ${ }^{[16]}$ In the same way hydrogenation of 7a led to perhydro-1,3-benzoxazine 10. Unfortunately, $\mathbf{1 0}$ turned out to be an oil that does not crystallize. However, $\mathbf{1 0}$ can be synthesized by another way that allows to determine without ambiguity its absolute stereochemistry (Scheme 3). The treatment of the ketone $\mathbf{1 1}^{[14]}$ with vinylmagnesium chloride provided the alcohol $\mathbf{1 2}$. The absolute configuration in the newly created stereocentre was assigned as $R$ in agreement with Eliel's reports. ${ }^{[13,14]}$ O-Allylation of alcohol 12 followed of RCM reaction and hydrogenation led to 10. 

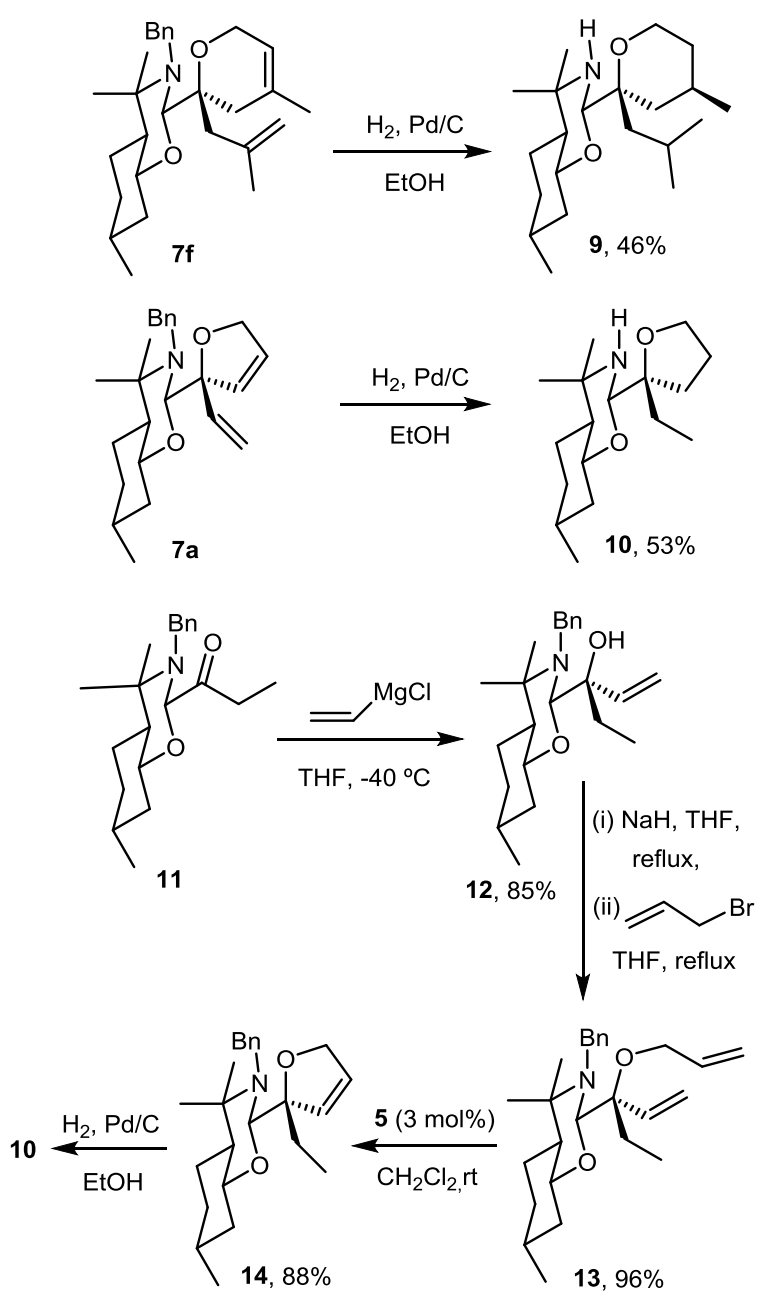

Scheme 3. Transformation of $7 \mathbf{f}$ and $7 \mathbf{a}$ into 9 and $\mathbf{1 0}$ and synthesis of $\mathbf{1 0}$ from $\mathbf{1 1}$

The utility of these DSRCM reactions was demonstrated by the transformation of some products into the final enantiopure dihydrofurans $15 \mathbf{a}, \mathbf{1 5}$ and 15d and dihydropyrans $\mathbf{1 5} \mathbf{e}-\mathbf{g}$ which was achieved in moderate chemical yield as depicted in Scheme 4 by hydrolysis of the N,O-acetal moiety by treatment with dilute $(\sim 2 \%)$ aqueous alcoholic hydrochloric acid $^{[13]}$ followed by reduction of the resulting aldehyde with sodium borohydride.

\section{Synthesis of chiral cyclic amines}

The starting trienic perhydro-1,3-benzoxazines 19a-e, 20a-e, and 21a-e were prepared in good to excellent yields, as summarized in Scheme 5. Addition of vinylic or allylic Grignard reagents to perhydrobenzoxazine $\mathbf{1 6}^{[12]}$ led to the alcohols 19a-c. Condensation of the amino alcohol $17^{[17]}$ with ethyl glyoxylate in toluene at reflux furnished a mixture of equatorial 18a and axial $\mathbf{1 8 b}$ esters in 76:24 ratio. Treatment of either $18 \mathbf{a}$ or the epimer $18 b$ with vinylic or allylic Grignard reagents gave the same equatorial carbinols 19d-e.

Since protection of the hydroxyl group could be important to achieve optimal chemical yield and diastereoselectivity in the DSRCM reaction of hydroxyl-containing trienic substrates, ${ }^{[18]}$ a set of

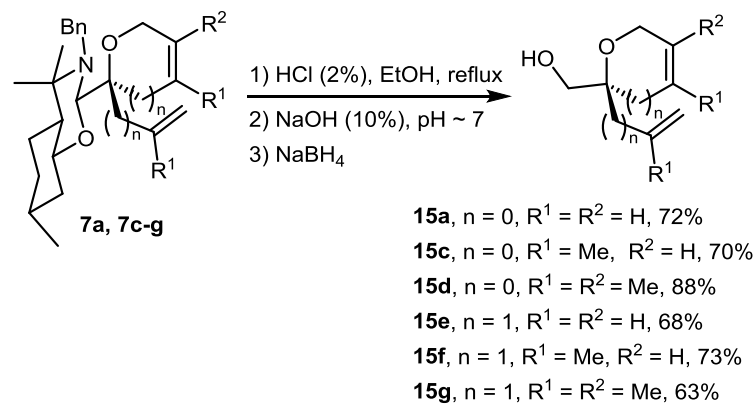

Scheme 4. Transformation of 7a,7c-g into dihydrofurans 15a,15c-d and dihydropyrans $15 \mathrm{e}-\mathrm{g}$.

substituted metathesis precursors was obtained from 19a-e introducing two hydroxyl protecting groups of different steric demand. The treatment of alcohols 19a-e with $\mathrm{NaH}$ and $\mathrm{MeI}$ in refluxing THF led the corresponding methyl ethers 20a-e with excellent chemical yield. On the other hand, conversion of the same alcohols into the corresponding TBDMS ethers 21a-e can be easily performed by reaction with tertbutyldimethylsilyl trifluoromethanesulfonate in the presence of 2,6-lutidine in dichloromethane at room temperature.

With the trienic substrates 19a-e, 20a-e and 21a-e in hand, we next examined the DSRCM reaction using the Grubbs' ruthenium carbene complex $\mathbf{5}$ and 6 as catalyst in $\mathrm{CH}_{2} \mathrm{Cl}_{2}$ as solvent. The results are summarized in Table 2.

First, the DSRCM reaction of trienic alcohol 19a, with monosubstituted olefinic appendages, in the presence of $\mathbf{5}$ was examined but, low conversion to cyclic products 22a and 23a and moderate diastereoselectivity was observed (entry 1 in Table 2). In the presence of the most active ruthenium carbene 6 a reversal of the stereoinduction occurred but the conversion and stereoselectivity remained moderate (entry 2).

The low conversion to cyclic products, even in the presence of high amounts of catalyst, could be due to coordination of the nitrogen atom of the perhydro1,3-benzoxazine to the ruthenium catalyst, resulting in deactivation of the metal complex through heteroatom-metal chelation. ${ }^{[19]}$ To avoid the formation of unreactive chelates we tested the protonation of perhydrobenzoxazine 19a. ${ }^{[20]}$ However, treatment of the hydrochloride salt of 19a with 4 mol$\%$ of Grubbs' catalyst $\mathbf{5}$, followed by a basic workup, gave the desired cyclic products 22a and 23a with only a slight improvement in the conversion and unfortunately, as an almost equal mixture of diastereomers (entry 3). To our delight, the ruthenium carbenes 5 and $\mathbf{6}$ promoted efficient ring closure of the methyl ether 20a and the silyl ether 21a (entries 4-7). The best results in terms of diastereoselectivity were achieved with the bulkier tert-butyldimethylsilyl protecting group. In the presence of the Grubbs' ruthenium carbene complex $\mathbf{6}$ perhydrobenzoxazine 21a led to 26a and 27a in a ratio of 13:87 (entry 7). 


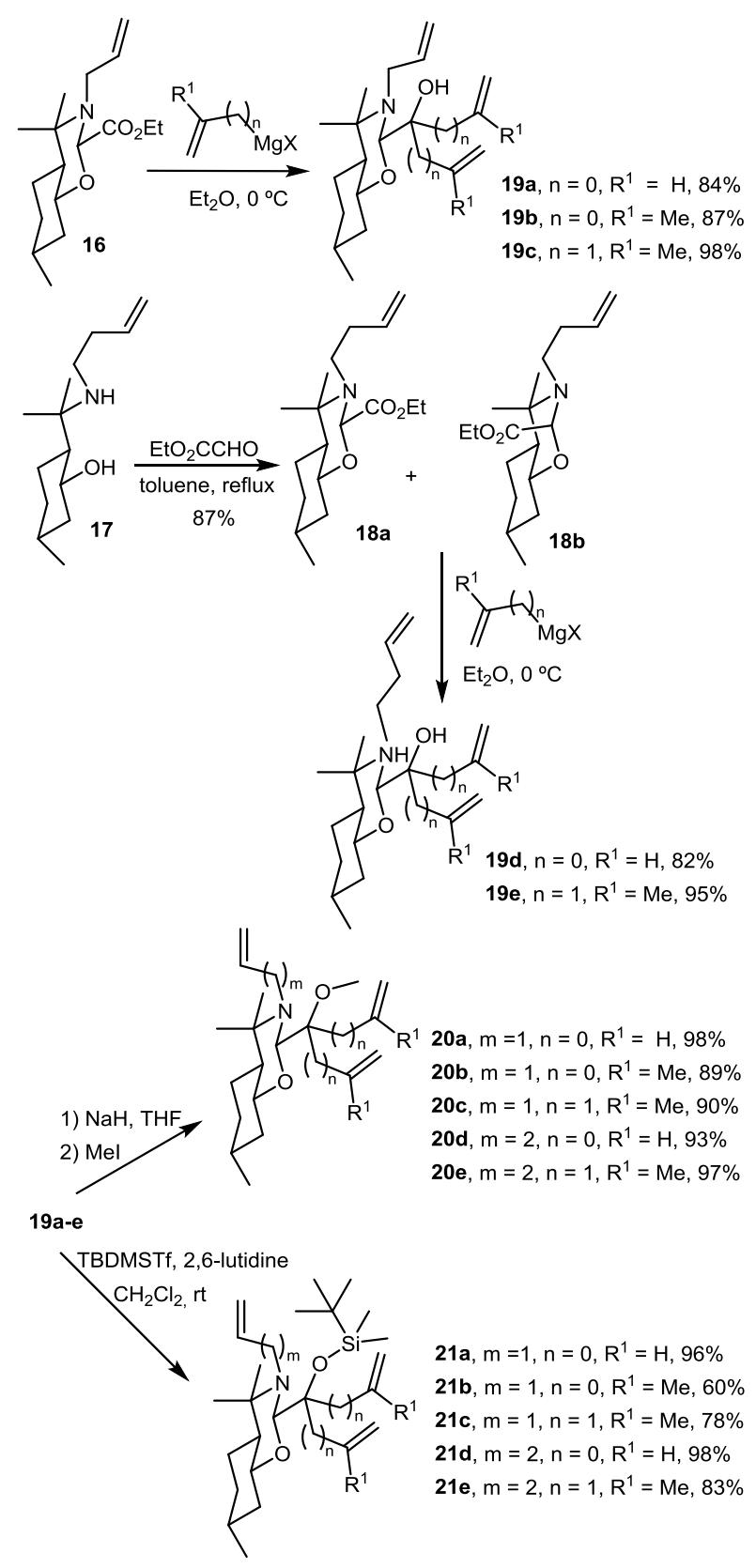

Scheme 5. Synthesis of perhydrobenzoxazines 19a-e, 20ae and 21a-e.

Neither the trienic alcohol $\mathbf{1 9 b}$ nor the corresponding silyl ether 21b with two 1,1disubstituted diastereotopic olefinic chains cyclized to the tetrahydropyridine ring (entries 8 and 11). The referable methyl ether $\mathbf{2 0 b}$ failed also to cyclize if the catalyst used is $\mathbf{5}$ (entry 9), however, in the presence of 6 , cyclized easily to a mixture of diastereomeric tetrahydropyridines $\mathbf{2 4 b}$ and $\mathbf{2 5 b}$ with a trisubstituted double bond with good diastereoselectivity (92:8, entry 10).

Similarly, tetrahydro- $1 H$-azepines can be prepared by DSRCM reaction of trienes $19 c, 20 c, 21 c, 20 d$ and 21d (entries 12-22). Again, the best results in terms of stereoselectivity were achieved when the hydroxyl group was protected as methyl ether if the diastereotopic olefin chains are 1,1-disubstituted entries 14 and 15) or as tert-butyldimethylsilyl ether if the diastereotopic olefin chains are monosubstituted (entries 21 and 22). In both cases, only the formation of a single diastereomer was detected by ${ }^{1} \mathrm{H}$ NMR spectra of the reaction mixture.

More interesting, the ruthenium carbene $\mathbf{6}$ catalyzed the DSRCM reaction of substrates $19 \mathbf{e}, \mathbf{2 0 e}$ and 21e providing the eight-membered cyclic amines (entries 23-25). As in 19c, the $\mathrm{OH}$ group in 19e does not seem to play a detrimental role in the diastereoselectivity (entry 23). Starting from the methyl ether 20e, the corresponding hexahydroazocine $\mathbf{2 4 e}$ with a trisubstituted double bond was obtained with good chemical yield and an excellent diastereoselectivity of 97: 3 (entry 24).

After isolation and purification by flash chromatography of the diastereomers formed in each reaction, the absolute configuration of the newly created stereocenters in 25a, 24c and $\mathbf{2 7 d}$ was established by X-ray diffraction studies. ${ }^{[16]}$ The absolute configuration of the newly created stereocenters in $\mathbf{2 4} \mathbf{e}$ was determined by its conversion into the aminomenthol derivatives $\mathbf{2 8}$ by nucleophilic ring opening of the $\mathrm{N}, \mathrm{O}$-acetal moiety by aluminum hydride (Scheme 6) and X-ray diffraction analysis. ${ }^{[16]}$ On the other hand, the NOESY spectra of $\mathbf{2 4 b}$ showed a cross signal for the hydrogen attached to the N,O-acetallic carbon of the oxazine ring and the hydrogens of the methoxy group indicating their cis relationship and that, therefore, the absolute configuration of the new stereocenter created at the DSRCM reaction is $R$.

On the other hand, O-methylation of 23a provided 25a, while the tert-butyldimethylsilyl ether cleavage of an inseparable mixture of 26a and 27a (13:87) with tetrabutylammonium fluoride in $\mathrm{THF}^{[21]}$ furnished a mixture of 22a and 23a in a 15:85 ratio, which allowed the assignation of the absolute stereochemistry of 25a, 27a and their epimers 24a and 26a respectively. In a similar way, the absolute configuration of the other RCM reaction products of Table 2 was assigned. Methylation of 22c provided $\mathbf{2 4 c}$, while deprotection of tert-butyldimethylsilyl ether in 27c furnished alcohol 23c. Deprotection of silyl ether in 27d followed by methylation of the resulting alcohol led 25d. Last, methylation of 22e provided 24e, while the tert-butyldimethylsilyl ether cleavage of an inseparable mixture of 26e and 27e in a $45: 55$ ratio furnished a mixture of the epimeric azocines 22e and 23e in 41:59 ratio.

Transformation of compounds 25a, 24b, 22c, 24c, 25d and 24e into the final enantiopure tetrahydropyridines $\mathbf{2 9}$ and $\mathbf{3 3}$, the tetrahydro- $1 H$ azepines 30, 32 and $\mathbf{3 4}$ and the hexahydroazocine $\mathbf{3 2}$ was performed in three steps, ${ }^{[22]}$ as depicted in Scheme 7. The reductive ring- opening of the N,Oacetal moiety by treatment with aluminium hydride generated in situ from lithium aluminium hydride and aluminium chloride, in THF at room temperature led to amino menthol derivatives in good to excellent yields. For a complete reduction of $\mathbf{2 4 b}$ was 
Table 2. DSRCM reaction of trienes 21-23.

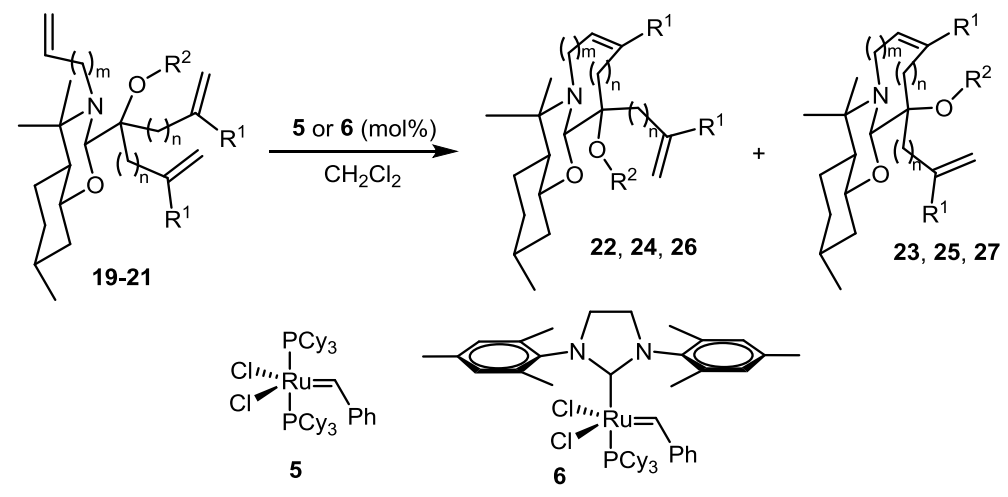

\begin{tabular}{|c|c|c|c|c|c|c|c|c|c|c|}
\hline Entry & & $\mathrm{m}$ & $\mathrm{n}$ & $\mathrm{R}^{1}$ & $\mathrm{R}^{2}$ & $T\left({ }^{\circ} \mathrm{C}\right)$ & $\begin{array}{l}\text { Catalyst } \\
(\mathrm{mol})^{\text {al] }}\end{array}$ & $t(\mathrm{~h})^{[\mathrm{a}]}$ & $\begin{array}{l}\text { Yield } \\
(\%)^{[b]}\end{array}$ & $d r(\%)^{[\mathrm{c}]}$ \\
\hline 1 & 19a & 1 & 0 & $\mathrm{H}$ & $\mathrm{H}$ & 40 & $5(2+2+2+2)$ & $15+15+15+10$ & $27^{[\mathrm{d}]}$ & 22a $(64) / 23 a(36)$ \\
\hline 2 & $19 a$ & 1 & 0 & $\mathrm{H}$ & $\mathrm{H}$ & 40 & $6(2+2+2)$ & $15+15+10$ & $38^{[\mathrm{d}]}$ & 22a $(35) / 23 \mathbf{a}(65)$ \\
\hline 3 & $19 \mathrm{a} \cdot \mathrm{HCl}$ & 1 & 0 & $\mathrm{H}$ & $\mathrm{H}$ & 20 & $5(2+2)$ & $15+50$ & $45^{[\mathrm{d}]}$ & $\mathbf{2 2} \mathbf{a}(48) / 23 \mathbf{a}(52)$ \\
\hline 4 & $\mathbf{2 0 a}$ & 1 & 0 & $\mathrm{H}$ & $\mathrm{Me}$ & 20 & $5(2+2)$ & $15+15$ & 90 & $\mathbf{2 4 a}(67) / 25 \mathbf{a}(33)$ \\
\hline 5 & 20a & 1 & 0 & $\mathrm{H}$ & $\mathrm{Me}$ & 20 & $6(2+2)$ & $15+10$ & 85 & $\mathbf{2 4 a}(27) / \mathbf{2 5} \mathbf{a}(73)$ \\
\hline 6 & $21 \mathrm{a}$ & 1 & 0 & $\mathrm{H}$ & TBDMS & 20 & $5(2+2)$ & $15+15$ & 83 & $\mathbf{2 6 a}(74) / 27 \mathbf{a}(26)$ \\
\hline 7 & $21 a$ & 1 & 0 & $\mathrm{H}$ & TBDMS & 20 & $6(2+2)$ & $15+15$ & 88 & 26a $(13) / 27 \mathbf{a}(87)$ \\
\hline 8 & $19 b$ & 1 & 0 & $\mathrm{Me}$ & $\mathrm{H}$ & 40 & $6(2+2)$ & $15+12$ & $---{ }^{[d]}$ & --- \\
\hline 9 & $20 b$ & 1 & 0 & $\mathrm{Me}$ & $\mathrm{Me}$ & 40 & $5(2+2)$ & $15+15+10$ & $-{ }_{--}^{[d]}$ & --- \\
\hline 10 & $20 b$ & 1 & 0 & $\mathrm{Me}$ & $\mathrm{Me}$ & 40 & $6(2+2)$ & $15+12$ & 81 & $\mathbf{2 4 b}(92) / \mathbf{2 5} \mathbf{b}(8)$ \\
\hline 11 & $21 b$ & 1 & 0 & $\mathrm{Me}$ & TBDMS & 40 & $6(2+2)$ & $15+12$ & $--^{\lfloor d\rfloor}$ & --- \\
\hline 12 & 19c & 1 & 1 & $\mathrm{Me}$ & $\mathrm{H}$ & 40 & $5(2+2+2+2)$ & $15+15+15+10$ & 78 & 22c $(92) / 23 c(8)$ \\
\hline 13 & $19 \mathrm{c}$ & 1 & 1 & $\mathrm{Me}$ & $\mathrm{H}$ & 40 & $6(2+2+2)$ & $15+15+10$ & 78 & 22c (73)/23c (27) \\
\hline 14 & 20c & 1 & 1 & $\mathrm{Me}$ & $\mathrm{Me}$ & 40 & $5(2+2)$ & $15+10$ & 94 & $\mathbf{2 4 c}(>98)^{[\mathrm{e}]}$ \\
\hline 15 & 20c & 1 & 1 & $\mathrm{Me}$ & $\mathrm{Me}$ & 40 & $6(2)$ & 12 & 92 & $\mathbf{2 4 c}(>98)^{[\mathrm{e}]}$ \\
\hline 16 & 21c & 1 & 1 & $\mathrm{Me}$ & TBDMS & 40 & $5(2+2)$ & $15+10$ & 90 & 26c $(35) / 27 \mathbf{c}(65)$ \\
\hline 17 & 21c & 1 & 1 & $\mathrm{Me}$ & TBDMS & 40 & $6(2+2)$ & $15+10$ & 92 & $\mathbf{2 6 c}(12) / 27 \mathbf{c}(88)$ \\
\hline 18 & 19d & 2 & 0 & $\mathrm{H}$ & $\mathrm{H}$ & 20 & $6(2+2)$ & $15+10$ & $-{ }_{--}^{[\mathrm{d}]}$ & -- \\
\hline 19 & 20d & 2 & 0 & $\mathrm{H}$ & $\mathrm{Me}$ & 20 & $5(2+2)$ & $15+10$ & 91 & $24 d(45) / 25 d(55)$ \\
\hline 20 & 20d & 2 & 0 & $\mathrm{H}$ & $\mathrm{Me}$ & 20 & $6(2)$ & 12 & 85 & $\mathbf{2 4 d}(30) / 25 d(70)$ \\
\hline 21 & 21d & 2 & 0 & $\mathrm{H}$ & TBDMS & 20 & $5(2+2)$ & $15+10$ & 45 & $\mathbf{2 7 d}(>98)^{[\mathrm{e}]}$ \\
\hline 22 & 21d & 2 & 0 & $\mathrm{H}$ & TBDMS & 20 & $6(2+2)$ & $15+10$ & 94 & 27d $(>98)^{[\mathrm{e}]}$ \\
\hline 23 & $19 e$ & 2 & 1 & $\mathrm{Me}$ & $\mathrm{H}$ & 40 & $6(2+2+2)$ & $15+15+10$ & 52 & $\mathbf{2 2 e}(90) / 23 \mathbf{e}(10)$ \\
\hline 24 & $20 \mathrm{e}$ & 2 & 1 & $\mathrm{Me}$ & $\mathrm{Me}$ & 40 & $6(3)$ & 4 & 90 & $24 \mathbf{e}(97) / 25 \mathbf{e}(3)$ \\
\hline 25 & 21e & 2 & 1 & $\mathrm{Me}$ & TBDMS & 40 & $6(3)$ & 4 & 91 & $26 \mathbf{e}(45) / 27 \mathbf{e}(55)$ \\
\hline
\end{tabular}

necessary to heat to $60{ }^{\circ} \mathrm{C}$ and under these reaction conditions demethylation of the tertiary methyl ether also occurs. Elimination of the menthol appendage in these derivatives was effected by oxidation with PCC in $\mathrm{CH}_{2} \mathrm{Cl}_{2}$ at room temperature to the corresponding 8 -aminomenthone derivatives followed by $\beta$ elimination by treatment with $\mathrm{KOH}$ in $\mathrm{H}_{2} \mathrm{O}-\mathrm{THF}-$ $\mathrm{MeOH}$ (Scheme 7).

\section{Conclusion}

In summary, the synthetic sequence described above constitutes a short and efficient procedure for the preparation of a variety of chiral oxygen- and nitrogen-containing heterocycles. The stereochemistry of the DSRCM reaction products is dependent on the length and position of the olefin chains in the perhydro-1,3-benzoxazine, the substitution pattern of the double bonds and the ruthenium carbene complex used as catalyst. The results collected in Table 1 and 2 show that the two catalysts, $\mathbf{5}$ and $\mathbf{6}$, behave differently both in terms of efficiency and diastereoselectivities, leading to the opposite epimers for the final heterocycle except in the case of very high selectivity were reached, where the major product obtained with catalyst 6 correspond to the one which is mainly produced by catalyst 5 

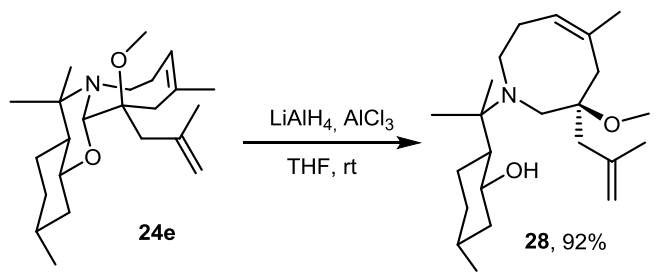

Scheme 6. Stereochemical correlation of compound $24 \mathbf{e}$.
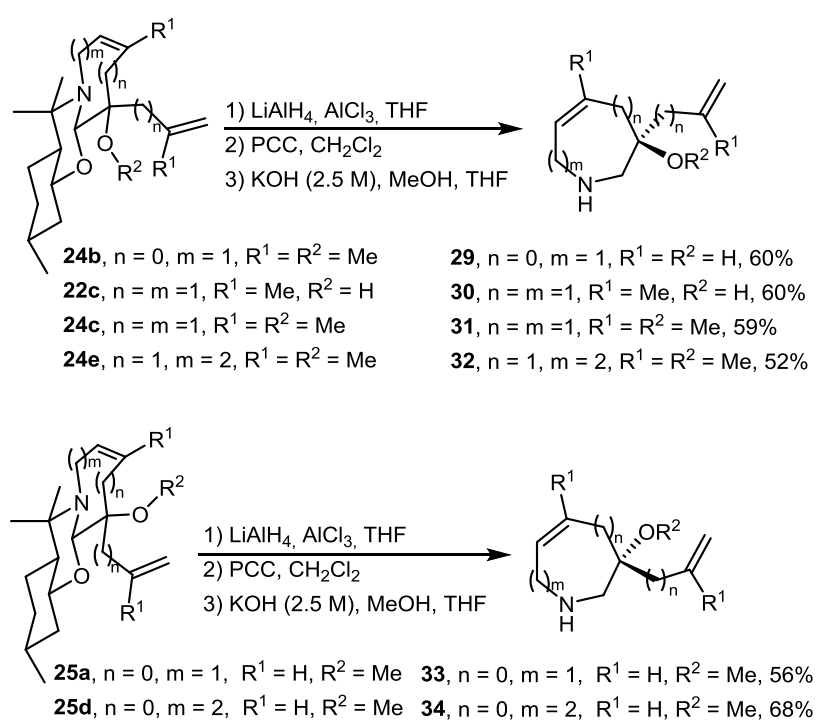

Scheme 7. Transformation of compounds 25a, 24b, 22c, 24c, 25d and 24e into the final enantiopure tetrahydropyridines $\mathbf{2 9}$ and $\mathbf{3 3}$, the tetrahydro- $1 H$-azepines 30, 32 and 34, and the hexahydroazocine $\mathbf{3 2}$.

when the diastereoselectivity is moderate. In addition, the results seem to show that the substrate plays a major role in the selection of the final stereoisomer as compared to the catalyst. Although in some cases only moderate diastereoselectivities are achieved in the cyclization, after separation of the diastereomers and removal of the chiral auxiliary enantiopure oxygen- and nitrogen-containing heterocycles are obtained.

\section{Experimental Section}

\section{General information}

All reactions were carried out in anhydrous solvents, under an argon atmosphere, and in oven-dried glassware. Commercial reagents were used without purification. ${ }^{1} \mathrm{H}$ NMR (300 or $400 \mathrm{MHz}$ ) and ${ }^{13} \mathrm{C}$ NMR $(75$ or $100 \mathrm{MHz})$ spectra were recorded in $\mathrm{CDCl}_{3}$. Chemical shifts for protons are reported in ppm from tetramethylsilane with the residual $\mathrm{CHCl}_{3}$ resonance as an internal reference. Chemical shifts for carbons are reported in ppm from tetramethylsilane and are referenced to the carbon resonance of the solvent. Data are reported as follows: chemical shift, multiplicity $(\mathrm{s}=$ singlet, $\mathrm{d}=$ doublet, $\mathrm{t}=$ triplet, $\mathrm{q}=$ quartet, $\mathrm{m}=$ multiplet, $\mathrm{br}=$ broad), coupling constants in hertz, and integration. High resolution mass spectrometry (HRMS) analysis was performed using a quadrupole spectrometer equipped with a TOF analyzer. Specific rotations were measured using a $5 \mathrm{~mL}$ cell with a $1 \mathrm{dm}$ path length and a sodium lamp, and the concentration is given in $\mathrm{g}$ per $100 \mathrm{~mL}$. Melting points were determined in open capillary tubes and are uncorrected. Flash chromatography was carried out using silica gel (230-240 mesh). TLC analysis was performed on glass-backed plates coated with silica gel 60 and an F254 indicator, and visualized either by UV irradiation or by staining with $I_{2}$ or phosphomolybdic acid solution. Chemical yields refer to pure isolated substances. Compounds $1,{ }^{13]} \mathbf{1 1},{ }^{[14]} \mathbf{1 6}^{[12]}$ and $17^{[17]}$ have been described previously. Compounds $\mathbf{2 b}, \mathbf{2 6 a}$ 27a, 25b, 26c, 23e, 26e and 27e could not be isolated and obtained pure after flash chromatography.

General procedure for the synthesis of benzoxazines 2 and 18. To a solution of $50 \mathrm{mmol}$ of $\mathbf{1}$ or $\mathbf{1 7} \mathrm{in} 150 \mathrm{~mL}$ of toluene was added $13 \mathrm{~mL}$ of a commercial solution of ethyl gyoxalate, $50 \%$ in toluene $(65 \mathrm{mmol})$. The solution was heated at reflux with a Dean-Stark trap until disappearance of the starting material (TLC, 12-24 h). The solvent was removed in vacuo and the residue was chromatographed on silica gel using hexanes/AcOEt as an eluent.

Ethyl

$(2 S, 4 a S, 7 R, 8 \mathrm{a} R)-3-$ benzyl-4,4,7trimethyloctahydro- $2 H$-benzo $[e][1,3]$ oxazine-2-

carboxylate (2a). This compound was obtained from 1 $(25.0 \mathrm{~g})$ and purified by flash chromatography (hexanes/ethyl acetate $15: 1$ ) to give $20.37 \mathrm{~g}$ of $\mathbf{2 a}$ and 9.33 $\mathrm{g}$ of a mixture of diastereomers $\mathbf{2 a}$ and $\mathbf{2 b}$ (90\% yield). $\mathbf{2} \mathbf{b}$ was quite configurationally unstable and quickly reverts to 2a. White solid. Mp $81-82^{\circ} \mathrm{C}$ (from hexane). $[\alpha]_{\mathrm{D}}{ }^{25}=-51.6$ $\left(c=0.7\right.$ ethyl acetate); ${ }^{1} \mathrm{H}$ NMR $\left(400 \mathrm{MHz}, \mathrm{CDCl}_{3}\right): \delta 0.93$ $(\mathrm{d}, J=6.5 \mathrm{~Hz}, 3 \mathrm{H}) ; 0.94-1.03(\mathrm{~m} 2 \mathrm{H}) ; 0.98(\mathrm{t}, J=7.2 \mathrm{~Hz}$, $3 \mathrm{H}) ; 1.03(\mathrm{~s}, 3 \mathrm{H}) ; 1.24(\mathrm{~m}, 1 \mathrm{H}) ; 1.28(\mathrm{~s}, 3 \mathrm{H}) ; 1.46(\mathrm{~m}$, $1 \mathrm{H}) ; 1.54-1.62(\mathrm{~m}, 2 \mathrm{H}) ; 1.70(\mathrm{~m}, 1 \mathrm{H}) ; 1.99(\mathrm{~m}, 1 \mathrm{H}) ; 3.54$ $\left(\mathrm{td}, J_{1}=10.5 \mathrm{~Hz}, J_{2}=4.0 \mathrm{~Hz}, 1 \mathrm{H}\right) ; 3.68\left(\mathrm{dq}, J_{1}=10.8 \mathrm{~Hz}\right.$, $\left.J_{2}=7.2 \mathrm{~Hz}, 1 \mathrm{H}\right) ; 3.85\left(\mathrm{dq}, J_{1}=10.8 \mathrm{~Hz}, J_{2}=7.2 \mathrm{~Hz}, 1 \mathrm{H}\right)$ $3.88(\mathrm{~d}, J=17.4 \mathrm{~Hz}, 1 \mathrm{H}) ; 3.95(\mathrm{~d}, J=17.4 \mathrm{~Hz}, 1 \mathrm{H}) ; 5.19$ $(\mathrm{s}, 1 \mathrm{H}) ; 7.12(\mathrm{t}, J=7.5 \mathrm{~Hz}, 1 \mathrm{H}) ; 7.22(\mathrm{t}, J=7.5 \mathrm{~Hz}, 2 \mathrm{H})$; $7.36(\mathrm{~d}, J=7.5 \mathrm{~Hz}, 2 \mathrm{H}) .{ }^{13} \mathrm{C}$ NMR $\left(100 \mathrm{MHz}^{\left.-\mathrm{CDCl}_{3}\right):}\right.$ $13.7\left(\mathrm{CH}_{3}\right) ; 20.7\left(\mathrm{CH}_{3}\right) ; 22.2\left(\mathrm{CH}_{3}\right) ; 24.9\left(\mathrm{CH}_{2}\right) ; 26.9$ $\left(\mathrm{CH}_{3}\right) ; 31.4(\mathrm{CH}) ; 34.9\left(\mathrm{CH}_{2}\right) ; 41.1\left(\mathrm{CH}_{2}\right) ; 45.6(\mathrm{CH}) ; 48.1$ $\left(\mathrm{CH}_{2}\right) ; 57.4(\mathrm{C}) ; 61.0\left(\mathrm{CH}_{2}\right) ; 76.3(\mathrm{CH}) ; 85.7(\mathrm{CH}) ; 126.1$ (CH); 127.3 (2 CH); 127.7 (2 CH); 142.2 (C); 168.8 (C). IR (Nujol dispersion): $3080,1750,1600,725,695 \mathrm{~cm}^{-1}$ HRMS calcd for $\mathrm{C}_{21} \mathrm{H}_{32} \mathrm{NO}_{3}[\mathrm{M}+\mathrm{H}]^{+} 346.2377$, found 346.2373

Ethyl $\quad(2 S, 4 a S, 7 R, 8 a R)-3$-(but-3-en-1-yl)-4,4,7trimethyloctahydro-2H-benzo $[e][1,3]$ oxazine-2-

carboxylate (18a). This compound was obtained from $\mathbf{1 7}$ $(12.0 \mathrm{~g})$ and purified by flash chromatography (hexanes/ethyl acetate 15:1). Yield: $10.83 \mathrm{~g}, 66 \%$. Colorless oil. $[\alpha]_{\mathrm{D}}{ }^{25}=-35.7\left(c=1.6 \mathrm{CH}_{2} \mathrm{Cl}_{2}\right) ;{ }^{\mathrm{H}} \mathrm{H} \mathrm{NMR}$ $\left(300 \mathrm{MHz}, \mathrm{CDCl}_{3}\right): \delta 0.88(\mathrm{~d}, J=6.5 \mathrm{~Hz}, 3 \mathrm{H}) ; 0.89-0.98$ $(\mathrm{m}, 2 \mathrm{H}) ; 1.11(\mathrm{~m}, 1 \mathrm{H}) ; 1.16(\mathrm{~s}, 6 \mathrm{H}) ; 1.28(\mathrm{t}, \mathrm{J}=7.2 \mathrm{~Hz}$, $3 \mathrm{H}) ; 1.35-1.48(\mathrm{~m}, 2 \mathrm{H}) ; 1.56-1.69(\mathrm{~m}, 2 \mathrm{H}) ; 1.93(\mathrm{~m}, 1 \mathrm{H})$; $2.08-2.17(\mathrm{~m}, 2 \mathrm{H}) ; 2.57(\mathrm{~m}, 1 \mathrm{H}) ; 2.71(\mathrm{~m}, 1 \mathrm{H}) ; 3.46(\mathrm{td}, J$ $\left.=10.6 \mathrm{~Hz}, J_{2}=4.0 \mathrm{~Hz}, 1 \mathrm{H}\right) ; 4.14-4.32(\mathrm{~m}, 2 \mathrm{H}) ; 4.88-4.98$ $(\mathrm{m}, 2 \mathrm{H}) ; 5.04(\mathrm{~s}, 1 \mathrm{H}) ; 5.79(\mathrm{~m}, 1 \mathrm{H}) .{ }^{13} \mathrm{C} \mathrm{NMR}(75 \mathrm{MHz}$ $\left.\mathrm{CDCl}_{3}\right): \delta 14.1\left(\mathrm{CH}_{3}\right) ; 19.9\left(\mathrm{CH}_{3}\right) ; 22.1\left(\mathrm{CH}_{3}\right) ; 24.9\left(\mathrm{CH}_{2}\right)$; $26.1\left(\mathrm{CH}_{3}\right) ; 31.2(\mathrm{CH}) ; 34.8\left(\mathrm{CH}_{2}\right) ; 37.2\left(\mathrm{CH}_{2}\right) ; 40.9$ $\left(\mathrm{CH}_{2}\right) ; 44.5\left(\mathrm{CH}_{2}\right) ; 45.6(\mathrm{CH}) ; 57.1(\mathrm{C}) ; 61.3\left(\mathrm{CH}_{2}\right) ; 76.0$ $(\mathrm{CH}) ; 85.7(\mathrm{CH}) ; 115.3\left(\mathrm{CH}_{2}\right) ; 136.1(\mathrm{CH}) ; 169.0(\mathrm{C})$. IR (película): $3075,1760,1640,910 \mathrm{~cm}^{-1}$. HRMS calcd for $\mathrm{C}_{18} \mathrm{H}_{32} \mathrm{NO}_{3}[\mathrm{M}+\mathrm{H}]^{+} 310.2377$, found 310.2373 .

Ethyl

$(2 R, 4 \mathrm{a} S, 7 R, 8 \mathrm{a} R)-3$-(but-3-en-1-yl)-4,4,7trimethyloctahydro- $2 H$-benzo $[e][1,3]$ oxazine-2-

carboxylate (18b). This compound was obtained from 17 $(12.0 \mathrm{~g})$ and purified by flash chromatography (hexanes/ethyl acetate 15:1). Yield: $3.42 \mathrm{~g}, 21 \%$. Colorless oil. $[\alpha]_{\mathrm{D}}{ }^{25}=+35.5\left(c=1.4 \mathrm{CH}_{2} \mathrm{Cl}_{2}\right) ;{ }^{1} \mathrm{H}$ NMR $(300 \mathrm{MHz}$, $\left.\mathrm{CDCl}_{3}\right): \delta 0.88-1.09(\mathrm{~m}, 3 \mathrm{H}) ; 0.93(\mathrm{~d}, J=6.5 \mathrm{~Hz}, 3 \mathrm{H})$; 
$1.01(\mathrm{~s}, 3 \mathrm{H}) ; 1.16(\mathrm{~s}, 3 \mathrm{H}) ; 1.30(\mathrm{t}, J=7.1 \mathrm{~Hz}, 3 \mathrm{H}) ; 1.39$ $1.60(\mathrm{~m}, 2 \mathrm{H}) ; 1.63-1.75(\mathrm{~m}, 2 \mathrm{H}) ; 1.99(\mathrm{~m}, 1 \mathrm{H}) ; 2.20-2.32$ $(\mathrm{m}, 2 \mathrm{H}) ; 2.88-3.01(\mathrm{~m}, 2 \mathrm{H}) ; 3.94\left(\mathrm{td}, J_{1}=10.6 \mathrm{~Hz}, J_{2}=4.0\right.$ $\mathrm{Hz}, 1 \mathrm{H}) ; 4.18-4.25(\mathrm{~m}, 2 \mathrm{H}) ; 4.80(\mathrm{~s}, 1 \mathrm{H}) ; 4.98-5.09(\mathrm{~m}$, $2 \mathrm{H}) ; 5.82(\mathrm{~m}, 1 \mathrm{H}) .{ }^{13} \mathrm{C} \mathrm{NMR}\left(75 \mathrm{MHz}, \mathrm{CDCl}_{3}\right): \delta 14.0$ $\left(\mathrm{CH}_{3}\right) ; 19.7\left(\mathrm{CH}_{3}\right) ; 22.1\left(\mathrm{CH}_{3}\right) ; 24.8\left(\mathrm{CH}_{2}\right) ; 27.5\left(\mathrm{CH}_{3}\right)$; $31.0(\mathrm{CH}) ; 34.7\left(\mathrm{CH}_{2}\right) ; 34.8\left(\mathrm{CH}_{2}\right) ; 41.2\left(\mathrm{CH}_{2}\right) ; 46.6$ $\left(\mathrm{CH}_{2}\right) ; 47.9(\mathrm{CH}) ; 55.3(\mathrm{C}) ; 60.7\left(\mathrm{CH}_{2}\right) ; 70.7(\mathrm{CH}) ; 83.7$ $(\mathrm{CH}) ; 115.6\left(\mathrm{CH}_{2}\right) ; 136.3(\mathrm{CH}) ; 172.6(\mathrm{C})$. IR (film): 3075 , 1740, 1640, $910 \mathrm{~cm}^{-1}$. HRMS calcd for $\mathrm{C}_{18} \mathrm{H}_{32} \mathrm{NO}_{3}[\mathrm{M}+$ $\mathrm{H}]^{+} 310.2377$, found 310.2377

\section{General procedure for addition of Grignard reagents to} $\mathbf{2 a}, \mathbf{1 1}, \mathbf{1 6}$ and the mixture of $\mathbf{1 8 a}$ and $\mathbf{1 8 b}$. A commercial solution of the appropriate vinyl- or allyl-Grignard derivative in ether or THF (46 mmol) was slowly added at $0{ }^{\circ} \mathrm{C}$ to a solution of the appropriate benzoxazine (20 mmol) in diethyl ether $(70 \mathrm{~mL})$. The mixture was stirred at this temperature until disappearance of the starting material (TLC, 20-45 $\mathrm{min}$ ). The reaction mixture was quenched with saturated ammonium chloride and the product was extracted with diethyl ether $(3 \times 40 \mathrm{~mL})$. The organic extracts were washed with brine, dried over anhydrous $\mathrm{MgSO}_{4}$, and the solvent was evaporated in vacuo. The residue was chromatographed on silica gel using hexanes/AcOEt as an eluent.

For ketone 11, the addition of vinyl magnesium choride (1.2 eq) was carried out at $-40{ }^{\circ} \mathrm{C}$ and THF was used as solvent instead of ether.

\section{3-((2S,4aS,7R,8aR)-3-benzyl-4,4,7-trimethyloctahydro-} $2 H$-benzo[e][1,3]oxazin-2-yl)penta-1,4-dien-3-ol (3a). This compound was obtained from $2 \mathbf{a}(7.0 \mathrm{~g})$ and purified by flash chromatography (hexanes/ethyl acetate 60:1). Yield: $5.96 \mathrm{~g}, 83 \%$. Colorless oil. $[\alpha]_{\mathrm{D}}{ }^{25}=+1.8(c=0.9$ EtOH); ${ }^{1} \mathrm{H}$ NMR (300 MHz, $\left.\mathrm{CDCl}_{3}\right): \delta 0.89-1.04(\mathrm{~m}, 2 \mathrm{H})$; $0.96(\mathrm{~s}, 3 \mathrm{H}) ; 0.97(\mathrm{~d}, J=6.5 \mathrm{~Hz}, 3 \mathrm{H}) ; 1.16(\mathrm{~m}, 1 \mathrm{H}) ; 1.32(\mathrm{~s}$, $3 \mathrm{H}) ; 1.32-1.64(\mathrm{~m}, 3 \mathrm{H}) ; 1.73(\mathrm{~m}, 1 \mathrm{H}) ; 1.96(\mathrm{~m}, 1 \mathrm{H}) ; 2.82$ $(\mathrm{s}, 1 \mathrm{H}) ; 3.57\left(\mathrm{dt}, J_{1}=10.5 \mathrm{~Hz}, J_{2}=4.0 \mathrm{~Hz}, 1 \mathrm{H}\right) ; 3.85(\mathrm{~d}, J$ $=18.5 \mathrm{~Hz}, 1 \mathrm{H}) ; 4.55(\mathrm{~d}, J=18.5 \mathrm{~Hz}, 1 \mathrm{H}) ; 4.72(\mathrm{~s}, 1 \mathrm{H})$; $4.99\left(\mathrm{dd}, J_{1}=10.6 \mathrm{~Hz}, J_{2}=1.6 \mathrm{~Hz}, 2 \mathrm{H}\right) ; 5.08\left(\mathrm{dd}, J_{1}=\right.$ $\left.17.3 \mathrm{~Hz}, J_{2}=1.6 \mathrm{~Hz}, 1 \mathrm{H}\right) ; 5.22\left(\mathrm{dd}, J_{1}=17.3 \mathrm{~Hz}, J_{2}=1.6\right.$ $\mathrm{Hz}, 1 \mathrm{H}) ; 6.02\left(\mathrm{ddd}, J_{1}=17.3 \mathrm{~Hz}, J_{2}=10.6 \mathrm{~Hz}, J_{3}=0.6 \mathrm{~Hz}\right.$, $1 \mathrm{H}) ; 6.12\left(\mathrm{dd}, J_{1}=17.3 \mathrm{~Hz}, J_{2}=10.6 \mathrm{~Hz}, 1 \mathrm{H}\right) ; 7.16(\mathrm{~m}$, $1 \mathrm{H}) ; 7.25-7.34(\mathrm{~m}, 4 \mathrm{H}) .{ }^{3} \mathrm{C} \mathrm{NMR}\left(75 \mathrm{MHz}, \mathrm{CDCl}_{3}\right): \delta$ $22.2\left(\mathrm{CH}_{3}\right) ; 22.9\left(\mathrm{CH}_{3}\right) ; 24.9\left(\mathrm{CH}_{2}\right) ; 26.6\left(\mathrm{CH}_{3}\right) ; 31.4$ (CH); $35.1\left(\mathrm{CH}_{2}\right) ; 41.3\left(\mathrm{CH}_{2}\right) ; 44.6(\mathrm{CH}) ; 46.7\left(\mathrm{CH}_{2}\right) ; 58.6$ (C); $76.1(\mathrm{C}) ; 77.9(\mathrm{CH}) ; 91.0(\mathrm{CH}) ; 112.7\left(\mathrm{CH}_{2}\right) ; 113.0$ $\left(\mathrm{CH}_{2}\right) ; 125.6(\mathrm{CH}) ; 126.6(2 \mathrm{CH}) ; 127.9(2 \mathrm{CH}) ; 139.8$ (CH); 140.2 (CH); 143.7 (C). IR (film): 3535 (broad), 3025 , $1605,760,720,695 \mathrm{~cm}^{-1}$. HRMS calcd for $\mathrm{C}_{23} \mathrm{H}_{34} \mathrm{NO}_{2}[\mathrm{M}$ $+\mathrm{H}]^{+} 356.2584$, found 356.2586

3-((2S,4aS,7R,8aR)-3-benzyl-4,4,7-trimethyloctahydro$2 H$-benzo[e][1,3]oxazin-2-yl)-2,4-dimethylpenta-1,4-

dien-3-ol (3b). This compound was obtained from 2a (7.0 g) and purified by flash chromatography (hexanes/ethyl acetate 60:1). Yield: $6.22 \mathrm{~g}, 88 \%$. Colorless oil. $[\alpha]_{\mathrm{D}}{ }^{25}=$ $+22.5\left(c=0.9 \mathrm{CH}_{2} \mathrm{Cl}_{2}\right)$. ${ }^{\mathrm{H}} \mathrm{NMR}\left(300 \mathrm{MHz}, \mathrm{CDCl}_{3}\right)$ : $\delta 0.77(\mathrm{~s}, 3 \mathrm{H}) ; 0.79-1.05(\mathrm{~m}, 2 \mathrm{H}) ; 0.94(\mathrm{~d}, J=6.5 \mathrm{~Hz}$, $3 \mathrm{H}) ; 1.14(\mathrm{~m}, 1 \mathrm{H}) ; 1.27(\mathrm{~m}, 1 \mathrm{H}) ; 1.31(\mathrm{~s}, 3 \mathrm{H}) ; 1.43-1.59$ $(\mathrm{m}, 2 \mathrm{H}) ; 1.67(\mathrm{~s}, 3 \mathrm{H}) ; 1.68(\mathrm{~m}, 1 \mathrm{H}) ; 1.77(\mathrm{~s}, 3 \mathrm{H}) ; 1.85(\mathrm{~m}$, $1 \mathrm{H}) ; 3.00(\mathrm{~s}, 1 \mathrm{H}) ; 3.61\left(\mathrm{dt}, J_{1}=10.6 \mathrm{~Hz}, J_{2}=3.9 \mathrm{~Hz}, 1 \mathrm{H}\right)$; $3.78(\mathrm{~d}, J=17.6 \mathrm{~Hz}, 1 \mathrm{H}) ; 4.66(\mathrm{t}, J=1.5 \mathrm{~Hz}, 1 \mathrm{H}) ; 4.92-$ $5.01(\mathrm{~m}, 4 \mathrm{H}) ; 5.23(\mathrm{~s}, 1 \mathrm{H}) ; 7.11(\mathrm{~m}, 1 \mathrm{H}) ; 7.19-7.27(\mathrm{~m}, 4$ $\mathrm{H}) .{ }^{13} \mathrm{C} N M R\left(75 \mathrm{MHz}, \mathrm{CDCl}_{3}\right): \delta 18.9\left(\mathrm{CH}_{3}\right) ; 19.4\left(\mathrm{CH}_{3}\right)$; $22.2\left(\mathrm{CH}_{3}\right) ; 22.5\left(\mathrm{CH}_{3}\right) ; 24.8\left(\mathrm{CH}_{2}\right) ; 27.3\left(\mathrm{CH}_{3}\right) ; 31.4$ $(\mathrm{CH})$; $35.1\left(\mathrm{CH}_{2}\right) ; 41.3\left(\mathrm{CH}_{2}\right) ; 43.3(\mathrm{CH}) ; 47.5\left(\mathrm{CH}_{2}\right) ; 58.6$ (C); $77.0(\mathrm{CH}) ; 80.5(\mathrm{C}) ; 85.2(\mathrm{CH}) ; 110.8\left(\mathrm{CH}_{2}\right) ; 113.1$ $\left(\mathrm{CH}_{2}\right) ; 124.9(\mathrm{CH}) ; 126.6(2 \mathrm{CH}) ; 127.1(2 \mathrm{CH}) ; 141.3(\mathrm{C})$; 145.5 (C); 147.6 (C). IR (film): 3550 (broad), 3085, 2868, 1640, 1605, 750, 720, $695 \mathrm{~cm}^{-1}$. HRMS calcd for $\mathrm{C}_{25} \mathrm{H}_{38} \mathrm{NO}_{2}[\mathrm{M}+\mathrm{H}]^{+}$384.2897, found 384.2900.

\section{3-( $(2 S, 4 \mathrm{a} S, 7 R, 8 \mathrm{a} R)-3$-benzyl-4,4,7-trimethyloctahydro-} 2H-benzo[e][1,3]oxazin-2-yl)-2,4-dimethylpenta-1,4dien-3-ol (3c). This compound was obtained from $\mathbf{2 a}(3.6$ g) and purified by flash chromatography (hexanes/ethyl acetate 60:1). Yield: $3.61 \mathrm{~g}, 90 \%$. White solid. Mp 82-84
${ }^{\circ} \mathrm{C}$ (from hexane). $[\alpha]_{\mathrm{D}}{ }^{25}=+62.0\left(c=0.8 \mathrm{CH}_{2} \mathrm{Cl}_{2}\right) ;{ }^{1} \mathrm{H}$ NMR $\left(300 \mathrm{MHz}, \mathrm{CDCl}_{3}\right): \delta 0.80-0.93(\mathrm{~m}, 2 \mathrm{H}) ; 0.87(\mathrm{~s}$ $3 \mathrm{H}) ; 0.94(\mathrm{~d}, J=6.5 \mathrm{~Hz}, 3 \mathrm{H}) ; 1.09(\mathrm{~m}, 1 \mathrm{H}) ; 1.25(\mathrm{~s}, 3 \mathrm{H})$; $1.52-1.68(\mathrm{~m}, 4 \mathrm{H}) ; 1.92(\mathrm{~m}, 1 \mathrm{H}) ; 2.24-2.40(\mathrm{~m}, 4 \mathrm{H}) ; 2.43$ $(\mathrm{s}, 1 \mathrm{H}) ; 3.51\left(\mathrm{td}, J_{1}=10.4 \mathrm{~Hz}, J_{2}=3.9 \mathrm{~Hz}, 1 \mathrm{H}\right) ; 3.88(\mathrm{~d}, J$ $=18.3 \mathrm{~Hz}, 1 \mathrm{H}) ; 4.50(\mathrm{~s}, 1 \mathrm{H}) ; 4.96(\mathrm{~d}, J=18.3 \mathrm{~Hz}, 1 \mathrm{H})$ $5.02-5.08(\mathrm{~m}, 4 \mathrm{H}) ; 5.78-6.00(\mathrm{~m}, 2 \mathrm{H}) ; 7.11(\mathrm{~m}, 1 \mathrm{H}) ; 7.19$ $7.22(\mathrm{~m}, 2 \mathrm{H}) ; 7.36-7.39(\mathrm{~m}, 2 \mathrm{H}) .{ }^{13} \mathrm{C}$ NMR $(75 \mathrm{MHz}$ $\left.\mathrm{CDCl}_{3}\right): \delta 22.3\left(\mathrm{CH}_{3}\right) ; 22.7\left(\mathrm{CH}_{3}\right) ; 24.9\left(\mathrm{CH}_{2}\right) ; 26.9\left(\mathrm{CH}_{3}\right)$ $31.5(\mathrm{CH}) ; 35.2\left(\mathrm{CH}_{2}\right) ; 38.7\left(\mathrm{CH}_{2}\right) ; 41.4\left(\mathrm{CH}_{2}\right) ; 43.6$ $\left(\mathrm{CH}_{2}\right) ; 43.9(\mathrm{CH}) ; 47.1\left(\mathrm{CH}_{2}\right) ; 58.4(\mathrm{C}) ; 75.6(\mathrm{C}) ; 77.6$ $(\mathrm{CH}) ; 88.0(\mathrm{CH}) ; 118.0\left(\mathrm{CH}_{2}\right) ; 118.1\left(\mathrm{CH}_{2}\right) ; 125.4(\mathrm{CH})$ $126.7(2 \mathrm{CH}) ; 127.8(2 \mathrm{CH}) ; 134.0(\mathrm{CH}) ; 134.2(\mathrm{CH})$; 144.4 (C). IR (Nujol dispersion): 3399 (broad), 3070, 1720 $1640,720,695650 \mathrm{~cm}^{-1}$. HRMS calcd for $\mathrm{C}_{25} \mathrm{H}_{38} \mathrm{NO}_{2}[\mathrm{M}$ $+\mathrm{H}]^{+}$384.2897, found 384.2905 .

4-((2S,4aS,7R,8aR)-3-benzyl-4,4,7-trimethyloctahydro$2 H$-benzo[e][1,3]oxazin-2-yl)-2,6-dimethylhepta-1,6-

dien-4-ol (3d). This compound was obtained from a mixture of $\mathbf{2 a}$ and $\mathbf{2 b}(7.0 \mathrm{~g})$ and purified by flash chromatography (hexanes/ethyl acetate 60:1). Yield: $7.64 \mathrm{~g}$, $92 \%$. Colorless oil. $[\alpha]_{\mathrm{D}}{ }^{25}=+14.3\left(c=1.4 \quad \mathrm{CH}_{2} \mathrm{Cl}_{2}\right) ;{ }^{1} \mathrm{H}$ NMR (300 MHz, CDCl $)$ : 0.79-0.98 (m, 2H); 0.89(s, 3H); $0.94(\mathrm{~d}, J=6.5 \mathrm{~Hz}, 3 \mathrm{H}) ; 1.11(\mathrm{~m}, 1 \mathrm{H}) ; 1.26(\mathrm{~s}, 3 \mathrm{H}) ; 1.26-$ $1.67(\mathrm{~m}, 4 \mathrm{H}) ; 1.68(\mathrm{~s}, 3 \mathrm{H}) ; 1.77(\mathrm{~s}, 3 \mathrm{H}) ; 1.85(\mathrm{~m}, 1 \mathrm{H})$ $2.20(\mathrm{~d}, J=14.9 \mathrm{~Hz}, 1 \mathrm{H}) ; 2.22(\mathrm{~d}, J=14.9 \mathrm{~Hz}, 1 \mathrm{H}) ; 2.32$ $(\mathrm{d}, J=14.9 \mathrm{~Hz}, 2 \mathrm{H}) ; 2.68(\mathrm{~s}, 1 \mathrm{H}) ; 3.49\left(\mathrm{td}, J_{1}=10.6 \mathrm{~Hz}\right.$ $\left.J_{2}=4.0 \mathrm{~Hz}, 1 \mathrm{H}\right) ; 3.91(\mathrm{~d}, J=18.5 \mathrm{~Hz}, 1 \mathrm{H}) ; 4.59(\mathrm{~s}, 1 \mathrm{H})$; $4.66-4.84(\mathrm{~m}, 5 \mathrm{H}) ; 7.13(\mathrm{~m}, 1 \mathrm{H}) ; 7.23-7.32(\mathrm{~m}, 2 \mathrm{H}) ; 7.39$ $7.41(\mathrm{~m}, 2 \mathrm{H}) \cdot{ }^{13} \mathrm{C}$ NMR $\left(75 \mathrm{MHz}, \mathrm{CDCl}_{3}\right): \delta 22.4\left(\mathrm{CH}_{3}\right)$; $22.8\left(\mathrm{CH}_{3}\right) ; 24.7\left(\mathrm{CH}_{3}\right) ; 24.7\left(\mathrm{CH}_{3}\right) ; 24.9\left(\mathrm{CH}_{2}\right) ; 26.9$ $\left(\mathrm{CH}_{3}\right) ; 31.5(\mathrm{CH}) ; 35.2\left(\mathrm{CH}_{2}\right) ; 41.3\left(\mathrm{CH}_{2}\right) ; 43.4\left(\mathrm{CH}_{2}\right)$; $44.0(\mathrm{CH}) ; 47.2\left(\mathrm{CH}_{2}\right) ; 47.4\left(\mathrm{CH}_{2}\right) ; 58.7(\mathrm{C}) ; 76.5(\mathrm{C})$ $77.0(\mathrm{CH}) ; 88.4(\mathrm{CH}) ; 113.5\left(\mathrm{CH}_{2}\right) ; 114.1\left(\mathrm{CH}_{2}\right) ; 125.5$ (CH); 126.8 (2 CH); 127.9 (2 CH); 143.4 (C); 143.8 (C); 147.6 (C). IR (film): 3545 (broad), 3070, 2870, 1640, 720, $695650 \mathrm{~cm}^{-1}$. HRMS calcd for $\mathrm{C}_{27} \mathrm{H}_{42} \mathrm{NO}_{2}[\mathrm{M}+\mathrm{H}]^{+}$ 412.3210, found 412.3212 .

\section{$(R)-3-((2 S, 4 a S, 7 R, 8 a R)-3-b e n z y l-4,4,7-$}

\section{trimethyloctahydro- $2 H$-benzo $[e][1,3]$ oxazin-2-yl)pent-}

1-en-3-ol (12). This compound was obtained from $11(2.5$ g) and purified by flash chromatography (hexanes/ethyl acetate 50:1). Yield: $2.30 \mathrm{~g}_{5} 85 \%$. White solid. Mp 118$119^{\circ} \mathrm{C}$ (from hexane). $[\alpha]_{\mathrm{D}}{ }^{25}=+8.9\left(c=1.1 \mathrm{CH}_{2} \mathrm{Cl}_{2}\right) .{ }^{1} \mathrm{H}$ NMR (300 MHz, $\left.\mathrm{CDCl}_{3}\right): \delta 0.82(\mathrm{t}, J=7.4 \mathrm{~Hz}, 3 \mathrm{H}) ; 0.88$ $(\mathrm{m}, 2 \mathrm{H}) ; 0.94(\mathrm{~d}, J=6.5 \mathrm{~Hz}, 3 \mathrm{H}) ; 0.96(\mathrm{~s}, 3 \mathrm{H}) ; 1.14(\mathrm{~m}$ $1 \mathrm{H}) ; 1.33(\mathrm{~s}, 3 \mathrm{H}) ; 1.43-1.68(\mathrm{~m}, 5 \mathrm{H}) ; 1.70(\mathrm{~m}, 1 \mathrm{H}) ; 1.91$ $(\mathrm{m}, 1 \mathrm{H}) ; 2.50(\mathrm{~s}, 1 \mathrm{H}) ; 3.53\left(\mathrm{td}, J_{1}=10.5 \mathrm{~Hz}, J_{2}=3.8 \mathrm{~Hz}\right.$ $1 \mathrm{H}) ; 3.83(\mathrm{~d}, J=18.6 \mathrm{~Hz}, 1 \mathrm{H}) ; 4.61(\mathrm{~d}, J=18.6 \mathrm{~Hz}, 1 \mathrm{H})$; $4.67(\mathrm{~s}, 1 \mathrm{H}, \mathrm{CH}) ; 4.94(\mathrm{~d}, J=17.1 \mathrm{~Hz}, 1 \mathrm{H}) ; 4.95(\mathrm{~d}, J=$ $11.1 \mathrm{~Hz}, 1 \mathrm{H}) ; 5.97\left(\mathrm{dd}, J_{1}=17.1 \mathrm{~Hz}, J_{2}=11.1 \mathrm{~Hz}, 1 \mathrm{H}\right)$ $7.13(\mathrm{~m}, 1 \mathrm{H}) ; 7.24-7.32(\mathrm{~m}, 4 \mathrm{H}) .{ }^{13} \mathrm{C} \mathrm{NMR}(75 \mathrm{MHz}$, $\left.\mathrm{CDCl}_{3}\right): \delta 7.53\left(\mathrm{CH}_{3}\right) ; 22.3\left(\mathrm{CH}_{3}\right) ; 22.9\left(\mathrm{CH}_{3}\right) ; 24.9\left(\mathrm{CH}_{2}\right)$ $26.6\left(\mathrm{CH}_{3}\right) ; 31.4\left(\mathrm{CH}\right.$ and $\left.\mathrm{CH}_{2}\right) ; 35.1\left(\mathrm{CH}_{2}\right) ; 41.3\left(\mathrm{CH}_{2}\right)$; $44.6(\mathrm{CH}) ; 46.8\left(\mathrm{CH}_{2}\right) ; 58.3(\mathrm{C}) ; 76.0(\mathrm{C}) ; 78.0(\mathrm{CH}) ; 90.9$ $(\mathrm{CH}) ; 113.1\left(\mathrm{CH}_{2}\right) ; 125.6(\mathrm{CH}) ; 126.3(2 \mathrm{CH}) ; 128.1(2$ $\mathrm{CH}) ; 140.8(\mathrm{CH}) ; 143.9$ (C). IR (film): 3540 (broad), 3025 , $1605,765,725,695 \mathrm{~cm}^{-1}$. HRMS calcd for $\mathrm{C}_{23} \mathrm{H}_{36} \mathrm{NO}_{2}[\mathrm{M}$ $+\mathrm{H}]^{+} 358.2741$, found 358.2746

\section{3-( $(2 S, 4 \mathrm{a} S, 7 R, 8 \mathrm{a} R)-3$-allyl-4,4,7-trimethyloctahydro-}

$2 H$-benzo[ $e][1,3]$ oxazin-2-yl)penta-1,4-dien-3-ol (19a). This compound was obtained from $16(10.0 \mathrm{~g})$ and purified by flash chromatography (hexanes/ethyl acetate 45:1). Yield: $8.70 \mathrm{~g}, 84 \%$. Colorless oil. $[\alpha]_{\mathrm{D}}{ }^{25}=-42.8(c=1.4$ $\left.\mathrm{CH}_{2} \mathrm{Cl}_{2}\right) \cdot{ }^{1} \mathrm{H}$ NMR $\left(300 \mathrm{MHz}, \mathrm{CDCl}_{3}\right): \delta 0.84-0.94(\mathrm{~m}$, $2 \mathrm{H}) ; 0.92(\mathrm{~d}, J=6.2 \mathrm{~Hz}, 3 \mathrm{H}) ; 1.06(\mathrm{~m}, 1 \mathrm{H}) ; 1.09(\mathrm{~s}, 3 \mathrm{H})$; $1.21(\mathrm{~s}, 3 \mathrm{H}) ; 1.43-1.51(\mathrm{~m}, 2 \mathrm{H}) ; 1.58(\mathrm{~m}, 1 \mathrm{H}) ; 1.69(\mathrm{~m}$ $1 \mathrm{H}) ; 1.90(\mathrm{~m}, 1 \mathrm{H}) ; 2.97(\mathrm{~s}, 1 \mathrm{H}) ; 3.24\left(\mathrm{dd}, J_{1}=18.3 \mathrm{~Hz}, J_{2}\right.$ $=4.9 \mathrm{~Hz}, 1 \mathrm{H}) ; 3.47\left(\mathrm{td}, J_{1}=10.6 \mathrm{~Hz}, J_{2}=3.9 \mathrm{~Hz}, 1 \mathrm{H}\right)$ $3.79\left(\mathrm{dd}, J_{1}=18.3 \mathrm{~Hz} J_{2}=4.9 \mathrm{~Hz}, 1 \mathrm{H}\right) ; 4.56(\mathrm{~s}, 1 \mathrm{H}) ; 4.97$ $5.17(\mathrm{~m}, 4 \mathrm{H}) ; 5.32\left(\mathrm{dd}, J_{1}=12.8 \mathrm{~Hz}, J_{2}=1.7 \mathrm{~Hz}, 1 \mathrm{H}\right)$; $5.38\left(\mathrm{dd}, J_{1}=12.8 \mathrm{~Hz}, J_{2}=1.7 \mathrm{~Hz}, 1 \mathrm{H}\right) ; 5.85(\mathrm{~m}, 1 \mathrm{H})$; $6.00\left(\mathrm{dd}, J_{1}=17.3 \mathrm{~Hz}, J_{2}=10.8 \mathrm{~Hz}, 1 \mathrm{H}\right) ; 6.15\left(\mathrm{dd}, J_{1}=\right.$ $\left.17.3 \mathrm{~Hz}, J_{2}=10.8 \mathrm{~Hz}, 1 \mathrm{H}\right),{ }^{13} \mathrm{C} \mathrm{NMR}\left(75 \mathrm{MHz} \mathrm{CDCl}_{3}\right): \delta$ $22.2\left(\mathrm{CH}_{3}\right) ; 22.8\left(\mathrm{CH}_{3}\right) ; 24.9\left(\mathrm{CH}_{2}\right) ; 26.3\left(\mathrm{CH}_{3}\right) ; 31.4$ $(\mathrm{CH}) ; 35.1\left(\mathrm{CH}_{2}\right) ; 41.2\left(\mathrm{CH}_{2}\right) ; 45.0(\mathrm{CH}) ; 45.3\left(\mathrm{CH}_{2}\right) ; 58.0$ (C); $75.7(\mathrm{C}) ; 77.9(\mathrm{CH}) ; 90.7(\mathrm{CH}) ; 112.5\left(2 \mathrm{CH}_{2}\right) ; 113.5$ 
$\left(\mathrm{CH}_{2}\right) ; 140.1(\mathrm{CH}) ; 140.6(\mathrm{CH}) ; 141.4(\mathrm{CH})$. IR (film): 3524 (broad), 3085, 1640, 820, 720, $635 \mathrm{~cm}^{-1}$. HRMS calcd for $\mathrm{C}_{19} \mathrm{H}_{32} \mathrm{NO}_{2}[\mathrm{M}+\mathrm{H}]^{+} 306.2428$, found 306.243

3-((2S,4aS,7R,8aR)-3-allyl-4,4,7-trimethyloctahydro$2 H$-benzo[ $e][1,3]$ oxazin-2-yl)-2,4-dimethylpenta-1,4dien-3-ol (19b). This compound was obtained from 16 $(10.0 \mathrm{~g})$ and purified by flash chromatography (hexanes/ethyl acetate 45:1). Yield: $9.80 \mathrm{~g}, 87 \%$. Colorless oil. $[\alpha]_{\mathrm{D}}{ }^{25}=+8.3\left(c=1.0 \mathrm{CH}_{2} \mathrm{Cl}_{2}\right) .{ }^{1} \mathrm{H}$ NMR $(300 \mathrm{MHz}$, $\left.\mathrm{CDCl}_{3}\right): \delta 0.90-1.00(\mathrm{~m}, 2 \mathrm{H}) ; 0.92(\mathrm{~d}, J=6.5 \mathrm{~Hz}, 3 \mathrm{H})$; $1.05(\mathrm{~m}, 1 \mathrm{H}) ; 1.07(\mathrm{~s}, 3 \mathrm{H}) ; 1.23(\mathrm{~s}, 3 \mathrm{H}) ; 1.43-1.62(\mathrm{~m}$, $3 \mathrm{H}) ; 1.69(\mathrm{~s}, 6 \mathrm{H}) ; 1.71(\mathrm{~m}, 1 \mathrm{H}) ; 1.88(\mathrm{~m}, 1 \mathrm{H}) ; 3.02(\mathrm{~s}$, $1 \mathrm{H}) ; 3.34\left(\mathrm{dd}, J_{1}=17.4 \mathrm{~Hz}, J_{2}=6.4 \mathrm{~Hz}, 1 \mathrm{H}\right) ; 3.53\left(\mathrm{td}, J_{1}=\right.$ $\left.10.6 \mathrm{~Hz}, J_{2}=3.9 \mathrm{~Hz}, 1 \mathrm{H}\right) ; 4.05\left(\mathrm{dd}, J_{1}=17.4 \mathrm{~Hz}, J_{2}=4.3\right.$ $\mathrm{Hz}, 1 \mathrm{H}) ; 4.79-4.98(\mathrm{~m}, 5 \mathrm{H}) ; 5.06(\mathrm{~s}, 1 \mathrm{H}) ; 5.21(\mathrm{~m}, 1 \mathrm{H})$; $5.89(\mathrm{~m}, 1 \mathrm{H}),{ }^{13} \mathrm{C} \mathrm{NMR}\left(75 \mathrm{MHz}, \mathrm{CDCl}_{3}\right): \delta 19.0\left(\mathrm{CH}_{3}\right)$; $19.1\left(\mathrm{CH}_{3}\right) ; 22.2\left(\mathrm{CH}_{3}\right) ; 22.6\left(\mathrm{CH}_{3}\right) ; 25.0\left(\mathrm{CH}_{2}\right) ; 27.1$ $\left(\mathrm{CH}_{3}\right) ; 31.4(\mathrm{CH}) ; 35.2\left(\mathrm{CH}_{2}\right) ; 41.4\left(\mathrm{CH}_{2}\right) ; 44.4(\mathrm{CH}) ; 46.9$ $\left(\mathrm{CH}_{2}\right) ; 58.3(\mathrm{C}) ; 77.0(\mathrm{CH}) ; 80.3(\mathrm{C}) ; 85.5(\mathrm{CH}) ; 110.9$ $\left(\mathrm{CH}_{2}\right) ; 111.3\left(\mathrm{CH}_{2}\right) ; 112.0\left(\mathrm{CH}_{2}\right) ; 142.8(\mathrm{CH}) ; 143.5(\mathrm{CH})$; 147,4 (CH). IR (film): 3550 (broad), 3085, 1640, 735, 610 $\mathrm{cm}^{-1}$. HRMS calcd for $\mathrm{C}_{21} \mathrm{H}_{36} \mathrm{NO}_{2}[\mathrm{M}+\mathrm{H}]^{+} 334.2741$, found 334.2748 .

\section{4-((2S,4aS,7R,8a $R)$-3-allyl-4,4,7-trimethyloctahydro- $2 H$-benzo $[e][1,3]$ oxazin-2-yl)-2,6-dimethylhepta-1,6- dien-4-ol (19c). This compound was obtained from 16 $(10.0 \mathrm{~g})$ and purified by flash chromatography (hexanes/ethyl acetate 45:1). Yield: $12.02 \mathrm{~g}, 98 \%$. Colorless oil. $[\alpha]_{\mathrm{D}}{ }^{25}=-25.3\left(c=1.8 \mathrm{CH}_{2} \mathrm{Cl}_{2}\right)$. ${ }^{\mathrm{P}} \mathrm{H}$ NMR $\left(300 \mathrm{MHz}, \mathrm{CDCl}_{3}\right): \delta 0.83-0.94(\mathrm{~m}, 2 \mathrm{H}) ; 0.92(\mathrm{~d}, J=6.5$ $\mathrm{Hz}, 3 \mathrm{H}) ; 0.99(\mathrm{~m}, 1 \mathrm{H}) ; 1.07(\mathrm{~s}, 3 \mathrm{H}) ; 1.17(\mathrm{~s}, 3 \mathrm{H}) ; 1.43-$ $1.68(\mathrm{~m}, 3 \mathrm{H}) ; 1.70-1.70-1.76(\mathrm{~m}, 2 \mathrm{H}) ; 1.81(\mathrm{~s}, 3 \mathrm{H}) ; 1.83$ $(\mathrm{s}, 3 \mathrm{H}) ; 2.18(\mathrm{~d}, J=14.6 \mathrm{~Hz}, 1 \mathrm{H}) ; 2.27(\mathrm{~d}, J=14.6 \mathrm{~Hz}$, $1 \mathrm{H}) ; 2.33(\mathrm{~d}, J=14.6 \mathrm{~Hz}, 1 \mathrm{H}) ; 2.45(\mathrm{~d}, J=14.6 \mathrm{~Hz}, 1 \mathrm{H})$; $2.69(\mathrm{~s}, 1 \mathrm{H}) ; 3.33\left(\mathrm{dd}, J_{1}=18.3 \mathrm{~Hz}, J_{2}=5.5 \mathrm{~Hz}, 1 \mathrm{H}\right) ; 3.41$ $\left(\mathrm{td}, J_{1}=10.6 \mathrm{~Hz}, J_{1} 2=3.9 \mathrm{~Hz}, 1 \mathrm{H}\right) ; 4.07\left(\mathrm{dd}, J_{1}=18.3 \mathrm{~Hz}\right.$, $\left.J_{2}=4.7 \mathrm{~Hz}, 1 \mathrm{H}\right) ; 4.44(\mathrm{~s}, 1 \mathrm{H}) ; 4.72-4.88(\mathrm{~m}, 4 \mathrm{H}) ; 4.99(\mathrm{dd}$, $\left.J_{1}=10.4 \mathrm{~Hz}, J_{2}=1.9 \mathrm{~Hz}, 1 \mathrm{H}\right) ; 5.15\left(\mathrm{dd}, J_{1}=17.5 \mathrm{~Hz}, J_{2}=\right.$ $1.9 \mathrm{~Hz}, 1 \mathrm{H}) ; 5.95(\mathrm{~m}, 1 \mathrm{H}) .{ }^{13} \mathrm{C} \mathrm{NMR}\left(75 \mathrm{MHz}, \mathrm{CDCl}_{3}\right): \delta$ $22.0\left(\mathrm{CH}_{3}\right) ; 22.5\left(\mathrm{CH}_{3}\right) ; 24.3\left(\mathrm{CH}_{3}\right) ; 24.4\left(\mathrm{CH}_{3}\right) ; 24.7$ $\left(\mathrm{CH}_{2}\right) ; 26.6\left(\mathrm{CH}_{3}\right) ; 31.1(\mathrm{CH}) ; 34.9\left(\mathrm{CH}_{2}\right) ; 41.0\left(\mathrm{CH}_{2}\right)$; $43.1\left(\mathrm{CH}_{2}\right) ; 43.9(\mathrm{CH}) ; 45.6\left(\mathrm{CH}_{2}\right) ; 47.0\left(\mathrm{CH}_{2}\right) ; 58.0(\mathrm{C})$; $75.9(\mathrm{CH}) ; 76.5(\mathrm{C}) ; 87.7(\mathrm{CH}) ; 113.0\left(\mathrm{CH}_{2}\right) ; 113.2\left(\mathrm{CH}_{2}\right) ;$ $114.0\left(\mathrm{CH}_{2}\right) ; 141.5(\mathrm{CH}) ; 143.2$ (C). IR (film): 3550 (broad), 3070, 1640, $735 \mathrm{~cm}^{-1}$. HRMS calcd for $\mathrm{C}_{23} \mathrm{H}_{40} \mathrm{NO}_{2}$ $[\mathrm{M}+\mathrm{H}]^{+}$362.3054, found 362.3057 .}

\section{3-((2S,4aS,7R,8a $R)-3$-(but-3-en-1-yl)-4,4,7-}

trimethyloctahydro- $2 H$-benzo $[e][1,3]$ oxazin-2-yl)penta1,4-dien-3-ol (19d). This compound was obtained from 18a $(9.30 \mathrm{~g})$ and purified by flash chromatography (hexanes/ethyl acetate 45:1). Yield: $7.90 \mathrm{~g}, 82 \%$. Colorless oil. $[\alpha]_{\mathrm{D}}{ }^{25}=-47.3\left(c=1.2 \mathrm{CH}_{2} \mathrm{Cl}_{2}\right)$. ${ }^{\mathrm{H}} \mathrm{NMR}(300 \mathrm{MHz}$, $\left.\mathrm{CDCl}_{3}\right): \delta 0.85-0.94(\mathrm{~m}, 2 \mathrm{H}) ; 0.91(\mathrm{~d}, J=6.6 \mathrm{~Hz}, 3 \mathrm{H})$; $1.06(\mathrm{~m}, 1 \mathrm{H}) ; 1.15(\mathrm{~s}, 3 \mathrm{H}) ; 1.18(\mathrm{~s}, 3 \mathrm{H}) ; 1.48-1.52(\mathrm{~m}$, $2 \mathrm{H}) ; 1.57(\mathrm{~m}, 1 \mathrm{H}) ; 1.69(\mathrm{~m}, 1 \mathrm{H}) ; 1.90(\mathrm{~m}, 1 \mathrm{H}) ; 2.08-2.21$ $(\mathrm{m}, 2 \mathrm{H}) ; 2.46(\mathrm{~m}, 1 \mathrm{H}) ; 3.05(\mathrm{~m}, 1 \mathrm{H}) ; 3.14(\mathrm{~s}, 1 \mathrm{H}) ; 3.44(\mathrm{td}$, $\left.J_{1}=10.5 \mathrm{~Hz}, J_{2}=4.0 \mathrm{~Hz}, 1 \mathrm{H}\right) ; 4.49(\mathrm{~s}, 1 \mathrm{H}) ; 4.91-4.99(\mathrm{~m}$, $2 \mathrm{H}) ; 5.07\left(\mathrm{dd}, J_{1}=10.5 \mathrm{~Hz}, J_{2}=1.8 \mathrm{~Hz}, 1 \mathrm{H}\right) ; 5.10\left(\mathrm{dd}, J_{1}=\right.$ $\left.10.5 \mathrm{~Hz}, J_{2}=1.8 \mathrm{~Hz}, 1 \mathrm{H}\right) ; 5.37\left(\mathrm{dd}, J_{1}=17.1 \mathrm{~Hz}, J_{2}=1.8\right.$ $\mathrm{Hz}, 1 \mathrm{H}) ; 5.48\left(\mathrm{dd}, J_{1}=17.1 \mathrm{~Hz}, J_{2}=1.8 \mathrm{~Hz}, 1 \mathrm{H}\right) ; 5.61(\mathrm{~m}$, $1 \mathrm{H}) ; 6.02\left(\mathrm{dd}, J_{1}=17.1 \mathrm{~Hz}, J_{2}=10.5 \mathrm{~Hz}, 1 \mathrm{H}\right) ; 6.15\left(\mathrm{dd}, J_{1}\right.$ $\left.=17.1 \mathrm{~Hz}, J_{2}=10.5 \mathrm{~Hz}, 1 \mathrm{H}\right) .{ }^{13} \mathrm{C} \mathrm{NMR}\left(75 \mathrm{MHz}, \mathrm{CDCl}_{3}\right)$ : $\delta 22.1\left(\mathrm{CH}_{3}\right) ; 22.9\left(\mathrm{CH}_{3}\right) ; 24.8\left(\mathrm{CH}_{2}\right) ; 26.1\left(\mathrm{CH}_{3}\right) ; 31.3$ $(\mathrm{CH}) ; 34.9\left(\mathrm{CH}_{2}\right) ; 37.4\left(\mathrm{CH}_{2}\right) ; 41.0\left(\mathrm{CH}_{2}\right) ; 42.2\left(\mathrm{CH}_{2}\right)$; $44.5(\mathrm{CH}) ; 57.8(\mathrm{C}) ; 74.9(\mathrm{C}) ; 77.9(\mathrm{CH}) ; 91.1(\mathrm{C}) ; 112.0$ $\left(\mathrm{CH}_{2}\right) ; 112.1\left(\mathrm{CH}_{2}\right) ; 115.2\left(\mathrm{CH}_{2}\right) ; 135.8(\mathrm{CH}) ; 139.4(\mathrm{CH}) ;$ 141,1 (CH). IR (film): 3460 (broad), 3075, 1640, 735, 630 $\mathrm{cm}^{-1}$. HRMS calcd for $\mathrm{C}_{20} \mathrm{H}_{34} \mathrm{NO}_{2}[\mathrm{M}+\mathrm{H}]^{+} 320.2584$, found 320.2584 .

4-((2S,4aS,7R,8aR)-3-(but-3-en-1-yl)-4,4,7-

trimethyloctahydro- $2 H$-benzo $[e][1,3]$ oxazin-2-yl)-2,6dimethylhepta-1,6-dien-4-ol (19e). This compound was obtained from a mixture of $\mathbf{1 8 a}$ and $18 \mathbf{b}(5.5 \mathrm{~g})$ and purified by flash chromatography (hexanes/ethyl acetate 50:1). Yield: $6.64 \mathrm{~g}, 95 \%$. Colorless oil. $[\alpha]_{\mathrm{D}}{ }^{25}=-26.5(c=$ $\left.1.4 \mathrm{CH}_{2} \mathrm{Cl}_{2}\right) .{ }^{1} \mathrm{H}$ NMR $\left(300 \mathrm{MHz}, \mathrm{CDCl}_{3}\right): \delta 0.85-0.96(\mathrm{~m}$, $2 \mathrm{H}) ; 0.92(\mathrm{~d}, J=6.5 \mathrm{~Hz}, 3 \mathrm{H}) ; 0.97-1.02(\mathrm{~m}, 1 \mathrm{H}) ; 1.14(\mathrm{~s}$ $6 \mathrm{H}) ; 1.21-1.51(\mathrm{~m}, 2 \mathrm{H}) ; 1.59(\mathrm{~m}, 1 \mathrm{H}) ; 1.68-1.79(\mathrm{~m}, 2 \mathrm{H})$; $1.84(\mathrm{~s}, 3 \mathrm{H}) ; 1.85(\mathrm{~s}, 3 \mathrm{H}) ; 2.212 .44(\mathrm{~m}, 6 \mathrm{H}) ; 2.63(\mathrm{~m}, 1 \mathrm{H})$ $2.77(\mathrm{~s}, 1 \mathrm{H}) ; 3.07(\mathrm{~m}, 1 \mathrm{H}) ; 3.39\left(\mathrm{td}, J_{1}=10.5 \mathrm{~Hz}, J_{2}=3.9\right.$ $\mathrm{Hz}, 1 \mathrm{H}) ; 4.46(\mathrm{~s}, 1 \mathrm{H}) ; 4.73-4.90(\mathrm{~m}, 4 \mathrm{H}) ; 4.95\left(\mathrm{dd}, J_{1}=\right.$ $\left.10.3 \mathrm{~Hz}, J_{2}=1.9 \mathrm{~Hz}, 1 \mathrm{H}\right) ; 5.02\left(\mathrm{dd}, J_{1}=17.2 \mathrm{~Hz}, J_{2}=1.9\right.$ $\mathrm{Hz}, 1 \mathrm{H}) ; 5.75(\mathrm{~m}, 1 \mathrm{H})$.$) . { }^{3} \mathrm{C} \mathrm{NMR}\left(75 \mathrm{MHz}^{\mathrm{C}} \mathrm{CDCl}_{3}\right): \delta$ $22.3\left(\mathrm{CH}_{3}\right) ; 22.9\left(\mathrm{CH}_{3}\right) ; 24.6\left(\mathrm{CH}_{3}\right) ; 24.7\left(\mathrm{CH}_{3}\right) ; 25.0$ $\left(\mathrm{CH}_{2}\right) ; 26.7\left(\mathrm{CH}_{3}\right) ; 31.4(\mathrm{CH}) ; 35.2\left(\mathrm{CH}_{2}\right) ; 38.0\left(\mathrm{CH}_{2}\right)$; $41.2\left(\mathrm{CH}_{2}\right) ; 42.9\left(\mathrm{CH}_{2}\right) ; 44.0\left(\mathrm{CH}_{2}\right) ; 44.3(\mathrm{CH}) ; 46.4\left(\mathrm{CH}_{2}\right)$; $58.4(\mathrm{C}) ; 75.7(\mathrm{C}) ; 77.0(\mathrm{CH}) ; 88.0(\mathrm{CH}) ; 113.7\left(\mathrm{CH}_{2}\right)$ $114.4\left(\mathrm{CH}_{2}\right) ; 115.2\left(\mathrm{CH}_{2}\right) ; 136.6(\mathrm{CH}) ; 143.4(\mathrm{C}) ; 143.7$ (C). IR (film): 3548 (broad), 3075, 1640, $730 \mathrm{~cm}^{-1}$. HRMS calcd for $\mathrm{C}_{24} \mathrm{H}_{42} \mathrm{NO}_{2}[\mathrm{M}+\mathrm{H}]^{+}$376.3210, found 376.3209.

General procedure for $\mathbf{O}$-allylation of alcohols $3 \mathrm{a}-\mathrm{d}$ and 12. To a solution of alcohol 3a-d or $\mathbf{1 2}(8.0 \mathrm{mmol})$ in THF $(40 \mathrm{~mL})$ was added $\mathrm{NaH}(60 \%$ dispersion in mineral oil $0.96 \mathrm{~g}, 24 \mathrm{mmol}$ ). The mixture was heated to reflux for 20 min and cooled to ambient temperature. Allyl bromide or methallyl bromide $(10.4 \mathrm{mmol})$ was added, and the mixture was again refluxed until the starting material was fully consumed as indicated by TLC. After aqueous workup, the THF was evaporated and the aqueous residue was extracted with diethyl ether $(3 \times 30 \mathrm{~mL})$. The organic extracts were washed with brine, dried over anhydrous $\mathrm{MgSO}_{4}$, and the solvent was evaporated in vacuo. The residue was chromatographed on silica gel using hexanes/AcOEt as an eluent.

$(2 S, 4 a S, 7 R, 8 \mathrm{a} R)$-2-(3-(allyloxy)penta-1,4-dien-3-yl)-3benzyl-4,4,7-trimethyloctahydro- $2 \mathrm{H}$ -

benzo $[e][1,3]$ oxazine $(\mathbf{4 a})$. This compound was obtained from 3a $(2.8 \mathrm{~g})$ and purified by flash chromatography (hexanes/ethyl acetate 60:1). Yield: $3.04 \mathrm{~g}, 98 \%$. Colorless oil. $[\alpha]_{\mathrm{D}}{ }^{25}=-18.5\left(c=1.6 \mathrm{CH}_{2} \mathrm{Cl}_{2}\right)$. ${ }^{1} \mathrm{H}$ NMR $(300 \mathrm{MHz}$ $\left.\mathrm{CDCl}_{3}\right): \delta 0.84-0.95(\mathrm{~m}, 2 \mathrm{H}) ; 0.86(\mathrm{~s}, 3 \mathrm{H}) ; 0.94(\mathrm{~d}, J=6.5$ $\mathrm{Hz}, 3 \mathrm{H}) ; 1.12(\mathrm{~m}, 1 \mathrm{H}) ; 1.25(\mathrm{~s}, 3 \mathrm{H}) ; 1.32-1.57(\mathrm{~m}, 3 \mathrm{H})$ $1.69(\mathrm{~m}, 1 \mathrm{H}) ; 1.96(\mathrm{~m}, 1 \mathrm{H}) ; 3.53\left(\mathrm{td}, J_{1}=10.5 \mathrm{~Hz}, J_{2}=3.9\right.$ $\mathrm{Hz}, 1 \mathrm{H}) ; 3.83(\mathrm{~d}, J=18.4 \mathrm{~Hz}, 1 \mathrm{H}) ; 3.89-4.00(\mathrm{~m}, 2 \mathrm{H})$ $4.59(\mathrm{~d}, J=18.4 \mathrm{~Hz}, 1 \mathrm{H}) ; 4.75(\mathrm{~s}, 1 \mathrm{H}) ; 5.04-5.17(\mathrm{~m}, 6 \mathrm{H})$ 5.84-6.06 (m, 3H); $7.11(\mathrm{~m}, 1 \mathrm{H}) ; 7.20-7.25(\mathrm{~m}, 2 \mathrm{H}) ; 7.34-$ $7.37(\mathrm{~m}, 2 \mathrm{H}) \cdot{ }^{13} \mathrm{C} \mathrm{NMR}\left(75 \mathrm{MHz}, \mathrm{CDCl}_{3}\right): \delta 22.2\left(\mathrm{CH}_{3}\right)$ $22.5\left(\mathrm{CH}_{3}\right) ; 22.8\left(\mathrm{CH}_{2}\right) ; 26.8\left(\mathrm{CH}_{3}\right) ; 31.4(\mathrm{CH}) ; 35.1$ $\left(\mathrm{CH}_{2}\right) ; 41.4\left(\mathrm{CH}_{2}\right) ; 44.1(\mathrm{CH}) ; 47.5\left(\mathrm{CH}_{2}\right) ; 58.2(\mathrm{C}) ; 64.4$ $\left(\mathrm{CH}_{2}\right), 77.6(\mathrm{CH}) ; 82.1(\mathrm{C}) ; 91.7(\mathrm{CH}) ; 114.8\left(2 \mathrm{CH}_{2}\right)$ $116.7^{\prime}\left(\mathrm{CH}_{2}\right) ; 125.2(\mathrm{CH}) ; 127.1(2 \mathrm{CH}) ; 127.4(2 \mathrm{CH})$ $135.9(\mathrm{CH}) ; 137.6(\mathrm{CH}) ; 137.9(\mathrm{CH}) ; 144.2(\mathrm{C})$. IR (film): $3080,2980,1635,1450,720,695 \mathrm{~cm}^{-1}$. HRMS calcd for $\mathrm{C}_{26} \mathrm{H}_{38} \mathrm{NO}_{2}[\mathrm{M}+\mathrm{H}]^{+}$396.2897, found 396.2900.

$(2 S, 4 a S, 7 R, 8 a R)-3-b e n z y l-4,4,7-t r i m e t h y l-2-(3-((2-)$ methylallyl)oxy)penta-1,4-dien-3-yl)octahydro- $2 \mathrm{H}$ benzo[e][1,3]oxazine $(4 \mathrm{~b})$. This compound was obtained from 3a $(2.8 \mathrm{~g})$ and purified by flash chromatography (hexanes/ethyl acetate 50:1). Yield: $2,75 \mathrm{~g}, 85 \%$. Colorless oil. $[\alpha]_{\mathrm{D}}{ }^{25}=-9.3\left(c=1.3 \mathrm{CH}_{2} \mathrm{Cl}_{2}\right)$. $\mathrm{H}$ NMR $(300 \mathrm{MHz}$, $\left.\mathrm{CDCl}_{3}\right): \delta 0.83-0.96(\mathrm{~m}, 2 \mathrm{H}) ; 0.84(\mathrm{~s}, 3 \mathrm{H}) ; 0.94(\mathrm{~d}, J=$ $6.5 \mathrm{~Hz}, 3 \mathrm{H}) ; 1.15(\mathrm{~m}, 1 \mathrm{H}) ; 1.26(\mathrm{~s}, 3 \mathrm{H}) ; 1.46-1.72(\mathrm{~m}, 4 \mathrm{H})$ $1.66(\mathrm{~s}, 3 \mathrm{H}) ; 1.96(\mathrm{~m}, 1 \mathrm{H}) ; 3.53\left(\mathrm{td}, J_{1}=10.5 \mathrm{~Hz}, J_{2}=3.9\right.$ $\mathrm{Hz}, 1 \mathrm{H}) ; 3.80-3.90(\mathrm{~m}, 3 \mathrm{H}) ; 4.58(\mathrm{~d}, J=18.0 \mathrm{~Hz}, 1 \mathrm{H})$ $4.78(\mathrm{~s}, 1 \mathrm{H}) ; 4.79(\mathrm{~d}, J=1.1 \mathrm{~Hz}, 1 \mathrm{H}) ; 5.03-5.31(\mathrm{~m}, 5 \mathrm{H})$ $5.89-6.06(\mathrm{~m}, 2 \mathrm{H}) ; 7.14(\mathrm{~m}, 1 \mathrm{H}) ; 7.22-7.24(\mathrm{~m}, 2 \mathrm{H}) ; 7.34$ $7.37(\mathrm{~m}, 2 \mathrm{H}) .{ }^{13} \mathrm{C}$ NMR $\left(75 \mathrm{MHz}, \mathrm{CDCl}_{3}\right): \delta 19.6\left(\mathrm{CH}_{3}\right)$ $22.2\left(\mathrm{CH}_{3}\right) ; 22.5\left(\mathrm{CH}_{3}\right) ; 24.8\left(\mathrm{CH}_{2}\right) ; 26.8\left(\mathrm{CH}_{3}\right) ; 31.3$ $(\mathrm{CH}) ; 35.1\left(\mathrm{CH}_{2}\right) ; 41.3\left(\mathrm{CH}_{2}\right) ; 44.0(\mathrm{CH}) ; 47.5\left(\mathrm{CH}_{2}\right) ; 58.2$ (C); $66.8\left(\mathrm{CH}_{2}\right)$ 77.5 (CH); $81.9(\mathrm{O}) ; 91.7(\mathrm{CH}) ; 109.6$ $\left(\mathrm{CH}_{2}\right) ; 114.7\left(\mathrm{CH}_{2}\right) ; 116.7\left(\mathrm{CH}_{2}\right) ; 125.1(\mathrm{CH}) ; 127.0$ $\mathrm{CH}) ; 127.4(2 \mathrm{CH}) ; 137.6(\mathrm{CH}) ; 137.9(\mathrm{CH}) ; 143.1(\mathrm{C})$ 144.2 (C). IR (film): $3080,2925,1650,1450,720,700 \mathrm{~cm}$ HRMS calcd for $\mathrm{C}_{27} \mathrm{H}_{40} \mathrm{NO}_{2}[\mathrm{M}+\mathrm{H}]^{+} 410.3054$, found 410.3054

$(2 S, 4 a S, 7 R, 8 a R)-2-(3-(a l l y l o x y)-2,4-d i m e t h y l p e n t a-1,4-$ dien-3-yl)-3-benzyl-4,4,7-trimethyloctahydro-2 $\mathrm{H}$ -

benzo[e] $]$ 1,3]oxazine $(\mathbf{4 c})$. This compound was obtained from $\mathbf{3 b}(3.0 \mathrm{~g})$ and purified by flash chromatography (hexanes/ethyl acetate 60:1). Yield: $2.90 \mathrm{~g}, 88 \%$. Colorless 
oil. $[\alpha]_{\mathrm{D}}{ }^{25}=-18.6\left(c=1.3 \mathrm{CH}_{2} \mathrm{Cl}_{2}\right) .{ }^{1} \mathrm{H}$ NMR $(300 \mathrm{MHz}$, $\left.\mathrm{CDCl}_{3}\right): \delta 0.78(\mathrm{~s}, 3 \mathrm{H}) ; 0.88-0.98(\mathrm{~m}, 2 \mathrm{H}) ; 0.96(\mathrm{~d}, J=6.6$ $\mathrm{Hz}, 3 \mathrm{H}) ; 1.04(\mathrm{~m}, 1 \mathrm{H}) ; 1.29(\mathrm{~s}, 3 \mathrm{H}) ; 1.54-1.55(\mathrm{~m}, 2 \mathrm{H})$; $1.57(\mathrm{~s}, 3 \mathrm{H}) ; 1.66-1.75(\mathrm{~m}, 2 \mathrm{H}) ; 1.78(\mathrm{~s}, 3 \mathrm{H}) ; 1.83(\mathrm{~m}$, $1 \mathrm{H}) ; 3.47\left(\mathrm{td}, J_{1}=10.5 \mathrm{~Hz}, J_{2}=3.9 \mathrm{~Hz}, 1 \mathrm{H}\right) ; 3.84(\mathrm{~d}, J=$ $17.5 \mathrm{~Hz}, 1 \mathrm{H}) ; 3.95\left(\mathrm{dd}, J_{1}=14.2 \mathrm{~Hz}, J_{2}=4.6 \mathrm{~Hz}, 1 \mathrm{H}\right)$; $4.44\left(\mathrm{dd}, J_{1}=14.2 \mathrm{~Hz}, J_{2}=4.6 \mathrm{~Hz}, 1 \mathrm{H}\right) ; 4.76(\mathrm{~s}, 1 \mathrm{H}) ; 5.01-$ $5.30(\mathrm{~m}, 7 \mathrm{H}) ; 5.93(\mathrm{~m}, 1 \mathrm{H}) ; 7.10(\mathrm{~m}, 1 \mathrm{H}) ; 7.18-7.23(\mathrm{~m}$, $2 \mathrm{H}) ; 7.27-7.34(\mathrm{~m}, 2 \mathrm{H}) .{ }^{13} \mathrm{C}$ NMR $\left(75 \mathrm{MHz}, \mathrm{CDCl}_{3}\right): \delta$ $19.4\left(\mathrm{CH}_{3}\right) ; 19.9\left(\mathrm{CH}_{3}\right) ; 22.3\left(\mathrm{CH}_{3}\right) ; 22.4\left(\mathrm{CH}_{3}\right) ; 24.9$ $\left(\mathrm{CH}_{2}\right) ; 27.5\left(\mathrm{CH}_{3}\right) ; 31.5(\mathrm{CH}) ; 35.3\left(\mathrm{CH}_{2}\right) ; 41.6\left(\mathrm{CH}_{2}\right)$; $43.3(\mathrm{CH}) ; 47.3\left(\mathrm{CH}_{2}\right) ; 58.7(\mathrm{C}) ; 66.6\left(\mathrm{CH}_{2}\right) ; 77.0(\mathrm{CH})$; $85.5(\mathrm{C}) ; 87.5(\mathrm{CH}) ; 113.8\left(\mathrm{CH}_{2}\right) ; 113.9\left(\mathrm{CH}_{2}\right) ; 115.3$ $\left(\mathrm{CH}_{2}\right) ; 124.8(\mathrm{CH}) ; 127.1(2 \mathrm{CH}) ; 127.1(2 \mathrm{CH}) ; 137.0$ (CH); 142.3 (C); 143.8 (C); 146.1 (C). IR (film): 3060, $2945,1640,1450,720,695 \mathrm{~cm}^{-1}$. HRMS calcd for $\mathrm{C}_{28} \mathrm{H}_{42} \mathrm{NO}_{2}[\mathrm{M}+\mathrm{H}]^{+}$424.3210, found 424.3214.

\section{(2S,4aS,7R,8aR)-3-benzyl-2-(2,4-dimethyl-3-((2-} methylallyl)oxy)penta-1,4-dien-3-yl)-4,4,7-

trimethyloctahydro- $2 H$-benzo $[e][1,3]$ oxazine $(4 d)$. This compound was obtained from 3b $(3.1 \mathrm{~g})$ and purified by flash chromatography (hexanes/ethyl acetate 60:1). Yield: $3.29 \mathrm{~g}, 93 \%$. Colorless oil. $[\alpha]_{\mathrm{D}}{ }^{25}=-20.9\left(c=1.0 \mathrm{CH}_{2} \mathrm{Cl}_{2}\right)$. ${ }^{1} \mathrm{H}$ NMR $\left(300 \mathrm{MHz}, \mathrm{CDCl}_{3}\right): \delta 0.76(\mathrm{~s}, 3 \mathrm{H}) ; 0.82-0.99(\mathrm{~m}$, $2 \mathrm{H}) ; 0.94(\mathrm{~d}, J=6.6 \mathrm{~Hz}, 3 \mathrm{H}) ; 1.12(\mathrm{~m}, 1 \mathrm{H}) ; 1.27(\mathrm{~s}, 3 \mathrm{H})$; $1.52-1.76(\mathrm{~m}, 4 \mathrm{H}) ; 1.54(\mathrm{~s}, 3 \mathrm{H}) ; 1.70(\mathrm{~s}, 3 \mathrm{H}) ; 1.75(\mathrm{~s}, 3 \mathrm{H})$; $1.88(\mathrm{~m}, 1 \mathrm{H}) ; 3.45\left(\mathrm{td}, J_{1}=10.5 \mathrm{~Hz}, J_{2}=3.9 \mathrm{~Hz}, 1 \mathrm{H}\right) ; 3.80$ $(\mathrm{d}, J=14.0 \mathrm{~Hz}, 1 \mathrm{H}) ; 3.82(\mathrm{~d}, J=17.1 \mathrm{~Hz}, 1 \mathrm{H}) ; 4.37(\mathrm{~d}, J$ $=14.0 \mathrm{~Hz}, 1 \mathrm{H}) ; 4.73(\mathrm{~d}, J=1.1 \mathrm{~Hz}, 2 \mathrm{H}) ; 4.92(\mathrm{~d}, J=1.1$ $\mathrm{Hz}, 1 \mathrm{H}) ; 5.07-5.17(\mathrm{~m}, 4 \mathrm{H}) ; 5.20(\mathrm{~s}, 1 \mathrm{H}) ; 7.07(\mathrm{~m}, 1 \mathrm{H})$; 7.15-7.20 (m, 2H); 7.24-7.31 (m, 2H). ). ${ }^{13} \mathrm{C}$ NMR $(75$ $\left.\mathrm{MHz}, \mathrm{CDCl}_{3}\right): \delta 19.4\left(\mathrm{CH}_{3}\right) ; 19.9\left(2 \mathrm{CH}_{3}\right) ; 22.3\left(2 \mathrm{CH}_{3}\right)$; $24.9\left(\mathrm{CH}_{2}\right) ; 27.4\left(\mathrm{CH}_{3}\right) ; 31.5(\mathrm{CH}) ; 35.3\left(\mathrm{CH}_{2}\right) ; 41.6$ $\left(\mathrm{CH}_{2}\right) ; 43.3(\mathrm{CH}) ; 47.2\left(\mathrm{CH}_{2}\right) ; 58.6(\mathrm{C}) ; 69.0\left(\mathrm{CH}_{2}\right) ; 77.0$ $(\mathrm{CH}) ; 85.3(\mathrm{C}) ; 87.7(\mathrm{CH}) ; 109.2\left(\mathrm{CH}_{2}\right) ; 113.9\left(\mathrm{CH}_{2}\right)$; $115.3\left(\mathrm{CH}_{2}\right) ; 124.8\left(\mathrm{CH}_{\mathrm{ar}}\right) ; 127.0(2 \mathrm{CH}) ; 127.1(2 \mathrm{CH})$; 142.4 (C); 143.8 (C); 144.1 (C); 146.0 (C). IR (film): 3065, $2915,1635,1450,720,695 \mathrm{~cm}^{-1}$. HRMS calcd for $\mathrm{C}_{29} \mathrm{H}_{44} \mathrm{NO}_{2}[\mathrm{M}+\mathrm{H}]^{+}$438.3367, found 438.3377 .

\section{(2S,4aS,7R,8aR)-2-(4-(allyloxy)hepta-1,6-dien-4-yl)-3-} benzyl-4,4,7-trimethyloctahydro- $2 H$ -

benzo[ $e][1,3]$ oxazine $(\mathbf{4 e})$. This compound was obtained from 3c $(3.1 \mathrm{~g})$ and purified by flash chromatography (hexanes/ethyl acetate 50:1). Yield: $3.15 \mathrm{~g}, 92 \%$. Colorless oil. $[\alpha]_{\mathrm{D}}{ }^{25}=+40.6\left(c=1.7 \mathrm{CH}_{2} \mathrm{Cl}_{2}\right) .{ }^{1} \mathrm{H} \mathrm{NMR}(300 \mathrm{MHz}$, $\left.\mathrm{CDCl}_{3}\right): \delta 0.82-1.01(\mathrm{~m}, 2 \mathrm{H}) ; 0.89(\mathrm{~s}, 3 \mathrm{H}) ; 0.96(\mathrm{~d}, J=6.5$ $\mathrm{Hz}, 3 \mathrm{H}) ; 1.12(\mathrm{~m}, 1 \mathrm{H}) ; 1.24(\mathrm{~s}, 3 \mathrm{H}) ; 1.43-1.62(\mathrm{~m}, 3 \mathrm{H})$; $1.70(\mathrm{~m}, 1 \mathrm{H}) ; 1.92(\mathrm{~m}, 1 \mathrm{H}) ; 2.27\left(\mathrm{dd}, J_{1}=14.0 \mathrm{~Hz}, J_{2}=6.9\right.$ $\mathrm{Hz}, 1 \mathrm{H}) ; 2.36$ (dd, $\left.J_{1}=14.0 \mathrm{~Hz}, J_{2}=7.4 \mathrm{~Hz}, 1 \mathrm{H}\right) ; 2.43$ (dd, $\left.J_{1}=14.4 \mathrm{~Hz}, J_{2}=8.5 \mathrm{~Hz}, 1 \mathrm{H}\right) ; 2.67\left(\mathrm{dd}, J_{1}=14.4 \mathrm{~Hz}, J_{2}=\right.$ $5.4 \mathrm{~Hz}, 1 \mathrm{H}) ; 3.37\left(\mathrm{td}, J_{1}=10.4 \mathrm{~Hz}, J_{2}=4.0 \mathrm{~Hz}, 1 \mathrm{H}\right) ; 3.92$ $(\mathrm{d}, J=18.4 \mathrm{~Hz}, 1 \mathrm{H}) ; 4.07\left(\mathrm{dd}, J_{1}=13.2 \mathrm{~Hz}, J_{2}=5.1 \mathrm{~Hz}\right.$, $1 \mathrm{H}) ; 4.43\left(\mathrm{dd}, J_{1}=13.2 \mathrm{~Hz}, J_{2}=5.3 \mathrm{~Hz}, 1 \mathrm{H}\right) ; 4.48(\mathrm{~s}, 1 \mathrm{H})$; $4.95-5.16(\mathrm{~m}, 7 \mathrm{H}) ; 5.75-5.98(\mathrm{~m}, 3 \mathrm{H}) ; 7.14(\mathrm{~m}, 1 \mathrm{H}) ; 7.21-$ $7.27(\mathrm{~m}, 2 \mathrm{H}) ; 7.31-7.45(\mathrm{~m}, 2 \mathrm{H}) .{ }^{13} \mathrm{C} \mathrm{NMR}(75 \mathrm{MHz}$, $\left.\mathrm{CDCl}_{3}\right): \delta 22.3\left(\mathrm{CH}_{3}\right) ; 22.4\left(\mathrm{CH}_{3}\right) ; 24.8\left(\mathrm{CH}_{2}\right) ; 26.7\left(\mathrm{CH}_{3}\right)$; $31.3\left(\mathrm{CH}_{2}\right) ; 35.1\left(\mathrm{CH}_{2}\right) ; 37.0\left(\mathrm{CH}_{2}\right) ; 39.4\left(\mathrm{CH}_{2}\right) ; 41.6$ $\left(\mathrm{CH}_{2}\right) ; 43.9(\mathrm{CH}) ; 46.6\left(\mathrm{CH}_{2}\right) ; 58.3(\mathrm{C}) ; 64.8\left(\mathrm{CH}_{2}\right) ; 77.5$ $(\mathrm{CH}) ; 80.3(\mathrm{C}) ; 89.9(\mathrm{CH}) ; 114.5\left(\mathrm{CH}_{2}\right) ; 117.6\left(2 \mathrm{CH}_{2}\right)$; $125.1(\mathrm{CH}) ; 126.5(2 \mathrm{CH}) ; 127.6(2 \mathrm{CH}) ; 134.2(\mathrm{CH})$; $134.6(\mathrm{CH}) ; 136.1(\mathrm{CH}) ; 144.8$ (C). IR (film): 3075,2925 , $1640,1450,725,695 \mathrm{~cm}^{-1}$. HRMS calcd for $\mathrm{C}_{28} \mathrm{H}_{42} \mathrm{NO}_{2}[\mathrm{M}$ $+\mathrm{H}]^{+} 424.3210$, found 424.3217 .

(2S,4aS,7R,8aR)-2-(4-(allyloxy)-2,6-dimethylhepta-1,6dien-4-yl)-3-benzyl-4,4,7-trimethyloctahydro-2H-

benzo[ $e][1,3]$ oxazine $(\mathbf{4 f})$. This compound was obtained from 3d (3.2 g) and purified by flash chromatography (hexanes/ethyl acetate 50:1). Yield: $3.37 \mathrm{~g}, 96 \%$. Colorless oil. $[\alpha]_{\mathrm{D}}{ }^{25}=-5.1\left(c=1.2 \mathrm{CH}_{2} \mathrm{Cl}_{2}\right) .{ }^{1} \mathrm{H}$ NMR $(300 \mathrm{MHz}$, $\left.\mathrm{CDCl}_{3}\right): \delta 0.86-0.98(\mathrm{~m}, 2 \mathrm{H}) ; 0.90(\mathrm{~s}, 3 \mathrm{H}) ; 0.94(\mathrm{~d}, J=6.5$ $\mathrm{Hz}, 3 \mathrm{H}) ; 1.11(\mathrm{~m}, 1 \mathrm{H}) ; 1.22(\mathrm{~s}, 3 \mathrm{H}) ; 1.35-1.56(\mathrm{~m}, 3 \mathrm{H})$; $1.60(\mathrm{~s}, 3 \mathrm{H}) ; 1.72(\mathrm{~m}, 1 \mathrm{H}) ; 1.82(\mathrm{~s}, 3 \mathrm{H}) ; 1.88(\mathrm{~m}, 1 \mathrm{H}) ; 2.23$ $(\mathrm{d}, J=15.0 \mathrm{~Hz}, 1 \mathrm{H}) ; 2.40(\mathrm{~d}, J=14.1 \mathrm{~Hz}, 1 \mathrm{H}) ; 2.56(\mathrm{~d}, J=$ $15.0 \mathrm{~Hz}, 1 \mathrm{H}) ; 2.69(\mathrm{~d}, J=14.1 \mathrm{~Hz}, 1 \mathrm{H}) ; 3.36\left(\mathrm{td}, J_{1}=10.5\right.$ $\left.\mathrm{Hz}, J_{2}=4.0 \mathrm{~Hz}, 1 \mathrm{H}\right) ; 3.92(\mathrm{~d}, J=18.2 \mathrm{~Hz}, 1 \mathrm{H}) ; 4.14(\mathrm{dd}$, $\left.J_{1}=13.2 \mathrm{~Hz}, J_{2}=4.9 \mathrm{~Hz}, 1 \mathrm{H}\right) ; 4.49\left(\mathrm{dd}, J_{1}=13.2 \mathrm{~Hz}, J_{2}=\right.$ $5.1 \mathrm{~Hz}, 1 \mathrm{H}) ; 4.58(\mathrm{~s}, 1 \mathrm{H}) ; 4.81(\mathrm{~s}, 2 \mathrm{H}) ; 4.90(\mathrm{~s}, 2 \mathrm{H}) ; 4.91-$ $5.06(\mathrm{~m}, 2 \mathrm{H}) ; 5.83(\mathrm{~m}, 1 \mathrm{H}) ; 7.11(\mathrm{~m}, 1 \mathrm{H}) ; 7.21-7.27(\mathrm{~m}$, $2 \mathrm{H}) ; 7.40-7.42(\mathrm{~m}, 2 \mathrm{H}) .{ }^{3} \mathrm{C} \mathrm{NMR}\left(75 \mathrm{MHz}, \mathrm{CDCl}_{3}\right): \delta$ $22.3\left(\mathrm{CH}_{3}\right) ; 22.6\left(\mathrm{CH}_{3}\right) ; 24.9\left(\mathrm{CH}_{2}\right) ; 25.1\left(2 \mathrm{CH}_{3}\right) ; 26.8$ $\left(\mathrm{CH}_{3}\right) ; 31.4(\mathrm{CH}) ; 35.2\left(\mathrm{CH}_{2}\right) ; 38.7\left(\mathrm{CH}_{2}\right) ; 41.5\left(\mathrm{CH}_{2}\right)$; $42.0\left(\mathrm{CH}_{2}\right) ; 44.0(\mathrm{CH}) ; 46.7\left(\mathrm{CH}_{2}\right) ; 58.6(\mathrm{C}) ; 64.7\left(\mathrm{CH}_{2}\right)$; $77.0(\mathrm{CH}) ; 82.0(\mathrm{C}) ; 89.8(\mathrm{CH}) ; 114.0\left(\mathrm{CH}_{2}\right) ; 114.4\left(\mathrm{CH}_{2}\right)$; $114.6\left(\mathrm{CH}_{2}\right) ; 125.1(\mathrm{CH}) ; 126.9(2 \mathrm{CH}) ; 127.6(2 \mathrm{CH})$; $136.4(\mathrm{CH}) ; 142.7$ (C); 143.6 (C); 145.0 (C). IR (film): $3075,2920,1640,1450,720,670 \mathrm{~cm}^{-1}$. HRMS calcd for $\mathrm{C}_{30} \mathrm{H}_{46} \mathrm{NO}_{2}[\mathrm{M}+\mathrm{H}]^{+} 452.3523$, found 452.3532 .

\section{(2S,4aS,7R,8aR)-3-benzyl-2-(2,6-dimethyl-4-((2-}

\section{methylallyl)oxy)hepta-1,6-dien-4-yl)-4,4,7-}

trimethyloctahydro- $2 H$-benzo $[e][1,3]$ oxazine $(4 \mathrm{~g})$. This compound was obtained from $3 \mathbf{d}(3.1 \mathrm{~g})$ and purified by flash chromatography (hexanes/ethyl acetate 55:1). Yield $3.26 \mathrm{~g}, 93 \%$. Colorless oil. $[\alpha]_{\mathrm{D}}{ }^{25}=+2.3\left(c=1.7 \mathrm{CH}_{2} \mathrm{Cl}_{2}\right)$.

${ }^{1} \mathrm{H} \mathrm{NMR}\left(300 \mathrm{MHz}, \mathrm{CDCl}_{3}\right): \delta 0.85-1.01(\mathrm{~m}, 2 \mathrm{H}) ; 0.90(\mathrm{~s}$, $3 \mathrm{H}) ; 0.94(\mathrm{~d}, J=6.5 \mathrm{~Hz}, 3 \mathrm{H}) ; 1.14(\mathrm{~m}, 1 \mathrm{H}) ; 1.22(\mathrm{~s}, 3 \mathrm{H})$; $1.35-1.61(\mathrm{~m}, 2 \mathrm{H}) ; 1.59(\mathrm{~s}, 3 \mathrm{H}) ; 1.66(\mathrm{~s}, 3 \mathrm{H}) ; 1.68(\mathrm{~m}$, $1 \mathrm{H}) ; 1.83(\mathrm{~s}, 3 \mathrm{H}) ; 1.89(\mathrm{~m}, 1 \mathrm{H}) ; 2.22(\mathrm{~d}, J=15.0 \mathrm{~Hz}, 1 \mathrm{H})$ $2.38(\mathrm{~d}, J=13.9 \mathrm{~Hz}, 1 \mathrm{H}) ; 2.57(\mathrm{~d}, J=15.0 \mathrm{~Hz}, 1 \mathrm{H}) ; 2.74$ $(\mathrm{d}, J=13.9 \mathrm{~Hz}, 1 \mathrm{H}) ; 3.35\left(\mathrm{td}, J_{1}=10.5 \mathrm{~Hz}, J_{2}=3.9 \mathrm{~Hz}\right.$, $1 \mathrm{H}) ; 3.91(\mathrm{~d}, J=18.3 \mathrm{~Hz}, 1 \mathrm{H}) ; 3.99(\mathrm{~d}, J=12.7 \mathrm{~Hz}, 1 \mathrm{H})$ $4.40(\mathrm{~d}, J=12.7 \mathrm{~Hz}, 1 \mathrm{H}) ; 4.59(\mathrm{~s}, 1 \mathrm{H}) ; 4.72(\mathrm{~d}, J=0.9$ $\mathrm{Hz}) ; 4.79(\mathrm{~s}, 1 \mathrm{H}) ; 4.80(\mathrm{~s}, 1 \mathrm{H}) ; 4.83(\mathrm{~s}, 1 \mathrm{H}) ; 4.90(\mathrm{~s}, 1 \mathrm{H})$; $5.00(\mathrm{~d}, J=18.3 \mathrm{~Hz}, 1 \mathrm{H}) ; 7.17(\mathrm{~m}, 1 \mathrm{H}) ; 7.31-7.40(\mathrm{~m}$ $2 \mathrm{H})$; 7.2-7.47 (m, 2H) ${ }^{13} \mathrm{C}$ NMR $\left(75 \mathrm{MHz}, \mathrm{CDCl}_{3}\right): \delta 20.1$ $\left(\mathrm{CH}_{3}\right) ; 22.3\left(\mathrm{CH}_{3}\right) ; 22.6\left(\mathrm{CH}_{3}\right) ; 24.9\left(\mathrm{CH}_{2}\right) ; 25.1\left(\mathrm{CH}_{3}\right)$;

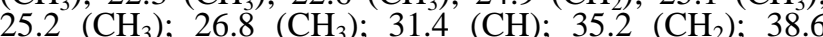
$\left(\mathrm{CH}_{2}\right) ; 41.6\left(\mathrm{CH}_{2}\right) ; 42.1\left(\mathrm{CH}_{2}\right) ; 44.0(\mathrm{CH}) ; 46.7\left(\mathrm{CH}_{2}\right)$; $58.5(\mathrm{C}) ; 67.3\left(\mathrm{CH}_{2}\right) ; 76.9(\mathrm{CH}) ; 82.0(\mathrm{C}) ; 89.8(\mathrm{CH})$ $110.0\left(\mathrm{CH}_{2}\right) ; 114.0\left(\mathrm{CH}_{2}\right) ; 114.7\left(\mathrm{CH}_{2}\right) ; 125.0(\mathrm{CH}) ; 126.9$ (2 CH); 127.5 (2 CH); 142.8 (C); 143.6 (C); 143.7 (C); 145.0 (C). IR (film): $3075,1640,720,695 \mathrm{~cm}^{-1}$. HRMS calcd for $\mathrm{C}_{31} \mathrm{H}_{48} \mathrm{NO}_{2}[\mathrm{M}+\mathrm{H}]^{+} 466.3680$, found 466.3687 .

\section{$(2 S, 4 a S, 7 R, 8 a R)-2-((R)-3-($ allyloxy $)$ pent-1-en-3-yl)-3-} benzyl-4, 4,7-trimethyloctahydro-2H -

benzo[e][1,3]oxazine (13). This compound was obtained from $12(2.2 \mathrm{~g})$ and purified by flash chromatography (hexanes/ethyl acetate 45:1). Yield: $2.35 \mathrm{~g}, 96 \%$. Colorless oil. $[\alpha]_{\mathrm{D}}{ }^{25}=-11.3\left(c=1.3 \mathrm{CH}_{2} \mathrm{Cl}_{2}\right) .{ }^{1} \mathrm{H}$ NMR $(300 \mathrm{MHz}$, $\left.\mathrm{CDCl}_{3}\right): \delta 0.8(\mathrm{t}, J=7.4 \mathrm{~Hz} 3 \mathrm{H},) ; 0.87(\mathrm{~s}, 3 \mathrm{H}) ; 0.89-1.11$ $(\mathrm{m}, 2 \mathrm{H}) 0.94(\mathrm{~d}, J=6.5 \mathrm{~Hz}, 3 \mathrm{H}) ; 1.17(\mathrm{~m}, 1 \mathrm{H}) ; 1.28(\mathrm{~s}, 3 \mathrm{H}$, $\mathrm{CH} 3) ; 1.42-1.63(\mathrm{~m}, 3 \mathrm{H}) ; 1.64-1.73(\mathrm{~m}, 3 \mathrm{H}) ; 1.90(\mathrm{~m}$, $1 \mathrm{H}) ; 3.46\left(\mathrm{td}, J_{1}=10.6 \mathrm{~Hz}, J_{2}=3.7 \mathrm{~Hz}, 1 \mathrm{H}\right) ; 3.82(\mathrm{~d}, J=$ $17.9 \mathrm{~Hz}, 1 \mathrm{H}) ; 4.14(\mathrm{~m}, 1 \mathrm{H}) ; 4.34(\mathrm{~m}, 1 \mathrm{H}) ; 4.76(\mathrm{~s}, 1 \mathrm{H})$; $4.84(\mathrm{~d}, J=17.9 \mathrm{~Hz}, 1 \mathrm{H}) ; 5.03-5.14(\mathrm{~m}, 2 \mathrm{H}) ; 5.14-5.25(\mathrm{~m}$, $2 \mathrm{H})$; 5.85-5.95 (m, 2H); 7.11 (m, 1H); 7.19-7.24 (m, 2H); $7.36-7.39(\mathrm{~m}, 2 \mathrm{H}),{ }^{13} \mathrm{C}$ NMR $\left(75 \mathrm{MHz} \mathrm{CDCl}_{3}\right): \delta 7.4$ $\left(\mathrm{CH}_{3}\right) ; 22.3\left(2 \mathrm{CH}_{3}\right) ; 24.9\left(\mathrm{CH}_{2}\right) ; 27.1\left(\mathrm{CH}_{3}\right) ; 28.1\left(\mathrm{CH}_{2}\right)$ $35.5(\mathrm{CH}) ; 35.2(\mathrm{CH} 2) ; 41.5\left(\mathrm{CH}_{2}\right) ; 44.1(\mathrm{CH}) ; 47.5$ $\left(\mathrm{CH}_{2}\right) ; 58.3(\mathrm{C}) ; 63.8\left(\mathrm{CH}_{2}\right), 77.8(\mathrm{CH}) ; 80.5(\mathrm{C}) ; 90.0$ $(\mathrm{CH}) ; 114.6\left(\mathrm{CH}_{2}\right) ; 115.0\left(\mathrm{CH}_{2}\right) ; 125.1(\mathrm{CH}) ; 127.0$ $\mathrm{CH}) ; 127.5(2 \mathrm{CH}) ; 136.3(\mathrm{CH}) ; 138.9(\mathrm{CH}) ; 144.2(\mathrm{C})$. IR (film): $3064,1452,721,695 \mathrm{~cm}^{-1}$. HRMS calcd for $\mathrm{C}_{26} \mathrm{H}_{40} \mathrm{NO}_{2}[\mathrm{M}+\mathrm{H}]^{+}$398.3054, found 398.3054

General procedure for methylation of alcohols 19a-e. To a solution of alcohols 19a-e $(9.0 \mathrm{mmol})$ in THF $(45$ $\mathrm{mL}$ ) was added $\mathrm{NaH}$ (60\% dispersion in mineral oil. $1.1 \mathrm{~g}$, $27 \mathrm{mmol}$ ). The mixture was heated to reflux for $20 \mathrm{~min}$ and cooled to ambient temperature. Iodomethane $(1.8 \mathrm{~mL}$ $28.8 \mathrm{mmol}$ ) was added, and the mixture was again refluxed until the starting material was fully consumed as indicated by TLC. After aqueous workup, the THF was evaporated and the aqueous residue was extracted with ethyl acetate (3 x $40 \mathrm{~mL}$ ). The organic extracts were washed with brine, dried over anhydrous $\mathrm{MgSO}_{4}$, and the solvent was evaporated in vacuo. The residue was chromatographed on silica gel using hexanes/AcOEt as an eluent.

(2S,4aS,7R,8a $R$ )-3-allyl-2-(3-methoxypenta-1,4-dien-3yl)-4,4,7-trimethyloctahydro- $2 H$-benzo $[e][1,3]$ oxazine (20a). This compound was obtained from 19a $(2.75 \mathrm{~g})$ and purified by flash chromatography (hexanes/ethyl acetate 50:1). Yield: $2.82 \mathrm{~g}, 98 \%$. Colorless oil. $[\alpha]_{\mathrm{D}}{ }^{25}=-49.2(c=$ $\left.1.2 \mathrm{CH}_{2} \mathrm{Cl}_{2}\right)$. $\mathrm{H}$ NMR $\left(300 \mathrm{MHz}, \mathrm{CDCl}_{3}\right): \delta 0.86-0.96(\mathrm{~m}$, 
$2 \mathrm{H}) ; 0.91(\mathrm{~d}, J=6.5 \mathrm{~Hz}, 3 \mathrm{H}) ; 1.07(\mathrm{~s}, 3 \mathrm{H}) ; 1.10(\mathrm{~m}, 1 \mathrm{H})$; $1.16(\mathrm{~s}, 3 \mathrm{H}) ; 1.21-1.57(\mathrm{~m}, 3 \mathrm{H}) ; 1.68(\mathrm{~m}, 1 \mathrm{H}) ; 1.93(\mathrm{~m}$, $1 \mathrm{H}) ; 3.19(\mathrm{~s}, 3 \mathrm{H}) ; 3.32\left(\mathrm{dd}, J_{l}=17.9 \mathrm{~Hz}, J_{2}=5.3 \mathrm{~Hz}, 1 \mathrm{H}\right)$; $3.45\left(\mathrm{td}, J_{l}=10.6 \mathrm{~Hz}, J_{2}=3.9 \mathrm{~Hz}, 1 \mathrm{H}\right) ; 3.83\left(\mathrm{dd}, J_{l}=17.9\right.$ $\left.\mathrm{Hz}, J_{2}=5.3 \mathrm{~Hz}, 1 \mathrm{H}\right) ; 4.57(\mathrm{~s}, 1 \mathrm{H}) ; 4.91\left(\mathrm{dd}, J_{l}=10.3 \mathrm{~Hz}\right.$, $\left.J_{2}=1.9 \mathrm{~Hz}, 1 \mathrm{H}\right) ; 5.03\left(\mathrm{dd}, J_{l}=17.4 \mathrm{~Hz}, J_{2}=1.9 \mathrm{~Hz}, 1 \mathrm{H}\right)$; 5.20-5.34 (m, 4H); 5.85-6.05 (m 3H). ${ }^{13} \mathrm{C}$ NMR $(75 \mathrm{MHz}$, $\left.\mathrm{CDCl}_{3}\right): \delta 22.1\left(\mathrm{CH}_{3}\right) ; 22.5\left(\mathrm{CH}_{3}\right) ; 24.9\left(\mathrm{CH}_{2}\right) ; 26.4\left(\mathrm{CH}_{3}\right)$; $31.3(\mathrm{CH}) ; 35.1\left(\mathrm{CH}_{2}\right) ; 41.3\left(\mathrm{CH}_{2}\right) ; 44.7(\mathrm{CH}) ; 46.4\left(\mathrm{CH}_{2}\right)$; $51.4\left(\mathrm{CH}_{3}\right) ; 57.8(\mathrm{C}) ; 77.4(\mathrm{CH}) ; 82.0(\mathrm{C}) ; 91.3(\mathrm{CH})$; $112.7\left(\mathrm{CH}_{2}\right) ; 115.2\left(\mathrm{CH}_{2}\right) ; 117.2\left(\mathrm{CH}_{2}\right) ; 136.9(\mathrm{CH}) ; 137.9$ (CH); 141.8 (CH). IR (film): $3085,1635,830 \mathrm{~cm}^{-1}$. HRMS calcd for $\mathrm{C}_{20} \mathrm{H}_{34} \mathrm{NO}_{2}[\mathrm{M}+\mathrm{H}]^{+} 320.2584$, found 320.2587 .

(2S,4aS,7R,8aR)-3-allyl-2-(3-methoxy-2,4dimethylpenta-1,4-dien-3-yl)-4,4,7-trimethyloctahydro$\mathbf{2 H}$-benzo[e][1,3] oxazine (20b). This compound was obtained from 19b $(3.0 \mathrm{~g})$ and purified by flash chromatography (hexanes/ethyl acetate 75:1). Yield: $2.80 \mathrm{~g}$, $89 \%$. Colorless oil. $[\alpha]_{\mathrm{D}}{ }^{25}=-5.8\left(c=1.0 \mathrm{CH}_{2} \mathrm{Cl}_{2}\right)$. 'H NMR $\left(300 \mathrm{MHz}, \mathrm{CDCl}_{3}\right): \delta 0.87-0.94(\mathrm{~m}, 2 \mathrm{H}) ; 0.92(\mathrm{~d}, J=6.5$ $\mathrm{Hz}, 3 \mathrm{H}) ; 1.05(\mathrm{~m}, \mathrm{H}) ; 1.06(\mathrm{~s}, 3 \mathrm{H}) ; 1.18(\mathrm{~s}, 3 \mathrm{H}) ; 1.26-1.55$ $(\mathrm{m}, 2 \mathrm{H}) ; 1.57(\mathrm{~s}, 3 \mathrm{H}) ; 1.65(\mathrm{~s}, 3 \mathrm{H}) ; 1.69(\mathrm{~m}, 1 \mathrm{H}) ; 1.86(\mathrm{~m}$, $1 \mathrm{H}) ; 3.31-3.48(\mathrm{~m}, 2 \mathrm{H}) ; 3.38(\mathrm{~s}, 3 \mathrm{H}) ; 4.16\left(\mathrm{dd}, J_{l}=17.3\right.$ $\left.\mathrm{Hz}, J_{2}=4.5 \mathrm{~Hz}, 1 \mathrm{H}\right) ; 4.77-4.94(\mathrm{~m}, 3 \mathrm{H}) ; 5.02(\mathrm{~s}, 1 \mathrm{H})$; 5.13-6.00 (m, 3H); $6.04(\mathrm{~m}, 1 \mathrm{H}) .{ }^{3} \mathrm{C}$ NMR $(75 \mathrm{MHz}$, $\left.\mathrm{CDCl}_{3}\right): \delta 19.6\left(\mathrm{CH}_{3}\right) ; 19.8\left(\mathrm{CH}_{3}\right) ; 22.3\left(\mathrm{CH}_{3}\right) ; 22.4\left(\mathrm{CH}_{3}\right)$; $25.1\left(\mathrm{CH}_{2}\right) ; 27.2\left(\mathrm{CH}_{3}\right) ; 31.5(\mathrm{CH}) ; 35.2\left(\mathrm{CH}_{2}\right) ; 41.7$ $\left(\mathrm{CH}_{2}\right) ; 44.4(\mathrm{CH}) ; 46.7\left(\mathrm{CH}_{2}\right) ; 53.9\left(\mathrm{CH}_{3}\right) ; 58.4(\mathrm{C}) ; 77.0$ $(\mathrm{CH}) ; 85.5(\mathrm{C}) ; 87.7(\mathrm{CH}) ; 111.0\left(\mathrm{CH}_{2}\right) ; 112.5\left(\mathrm{CH}_{2}\right)$; $115.6\left(\mathrm{CH}_{2}\right) ; 143.4(\mathrm{C}) ; 143.9(\mathrm{C}) ; 144.3(\mathrm{CH})$. IR (film): $3070,1640,830,730 \mathrm{~cm}^{-1}$. HRMS calcd for $\mathrm{C}_{22} \mathrm{H}_{38} \mathrm{NO}_{2}[\mathrm{M}$ $+\mathrm{H}]^{+}$348.2897, found 348.2900.

$(2 S, 4 a S, 7 R, 8 \mathrm{a} R)$-3-allyl-2-(4-methoxy-2,6dimethylhepta-1,6-dien-4-yl)-4,4,7-trimethyloctahydro$\mathbf{2 H}$-benzo[e][1,3] oxazine $(\mathbf{2 0 c})$. This compound was obtained from 19c $(3.0 \mathrm{~g})$ and purified by flash chromatography (hexanes/ethyl acetate 75:1). Yield: $2.80 \mathrm{~g}$, $90 \%$. Colorless oil. $[\alpha]_{\mathrm{D}}{ }^{25}=-41.4\left(c=1.0 \mathrm{CH}_{2} \mathrm{Cl}_{2}\right) .{ }^{1} \mathrm{H}$ NMR $\left(300 \mathrm{MHz}, \mathrm{CDCl}_{3}\right): \delta 0.86-0.96(\mathrm{~m}, 2 \mathrm{H}) ; 0.91(\mathrm{~d}, J=$ $6.5 \mathrm{~Hz}, 3 \mathrm{H}) ; 1.05(\mathrm{~m}, 1 \mathrm{H}) ; 1.09(\mathrm{~s}, 3 \mathrm{H}) ; 1.11(\mathrm{~s}, 3 \mathrm{H}) ; 1.42-$ $1.59(\mathrm{~m}, 3 \mathrm{H}) ; 1.70(\mathrm{~m}, 1 \mathrm{H}) ; 1.78(\mathrm{~s}, 3 \mathrm{H}) ; 1.82(\mathrm{~s}, 3 \mathrm{H})$; $1.84(\mathrm{~m}, 1 \mathrm{H}) ; 2.21(\mathrm{~d}, J=15.0 \mathrm{~Hz}, 1 \mathrm{H}) ; 2.42(\mathrm{~d}, J=14.2$ $\mathrm{Hz}, 1 \mathrm{H}) ; 2.57$ (d, $J=15.0 \mathrm{~Hz}, 1 \mathrm{H}) ; 2.64(\mathrm{~d}, J=14.2 \mathrm{~Hz}$, $1 \mathrm{H}) ; 3.28\left(\mathrm{td}, J_{l}=10.4 \mathrm{~Hz}, J_{2}=3.9 \mathrm{~Hz}, 1 \mathrm{H}\right) ; 3.41(\mathrm{~s}, 3 \mathrm{H})$; $3.43\left(\mathrm{dd}, J_{l}=17.7 \mathrm{~Hz}, J_{2}=5.4 \mathrm{~Hz}, 1 \mathrm{H}\right) ; 4.11\left(\mathrm{dd}, J_{l}=17.7\right.$ $\left.\mathrm{Hz}, J_{2}=5.4 \mathrm{~Hz}, 1 \mathrm{H}\right) ; 4.41(\mathrm{~s}, 1 \mathrm{H}) ; 4.80-4.86(\mathrm{~m}, 4 \mathrm{H}) ; 4.94$ $\left(\mathrm{dd}, J_{l}=10.3 \mathrm{~Hz}, J_{2}=1.9 \mathrm{~Hz}, 1 \mathrm{H}\right) ; 5.10\left(\mathrm{dd}, J_{l}=17.3 \mathrm{~Hz}\right.$, $\left.J_{2}=1.9 \mathrm{~Hz}, 1 \mathrm{H}\right) ; 6.04(\mathrm{~m}, 1 \mathrm{H}) .{ }^{13} \mathrm{C} \mathrm{NMR}(75 \mathrm{MHz}$, $\left.\mathrm{CDCl}_{3}\right): \delta 22.2\left(\mathrm{CH}_{3}\right) ; 22.4\left(\mathrm{CH}_{3}\right) ; 24.8\left(\mathrm{CH}_{3}\right) ; 24.9\left(\mathrm{CH}_{2}\right)$; $25.2\left(\mathrm{CH}_{3}\right) ; 26.8\left(\mathrm{CH}_{3}\right) ; 31.4(\mathrm{CH}) ; 35.2\left(\mathrm{CH}_{2}\right) ; 38.3$ $\left(\mathrm{CH}_{2}\right) ; 41.4\left(\mathrm{CH}_{2}\right) ; 41.5\left(\mathrm{CH}_{2}\right) ; 44.3(\mathrm{CH}) ; 46.0\left(\mathrm{CH}_{2}\right)$; $51.2\left(\mathrm{CH}_{3}\right) ; 58.3(\mathrm{C}) ; 76.6(\mathrm{CH}) ; 81.5(\mathrm{C}) ; 89.6(\mathrm{CH})$; $112.6\left(\mathrm{CH}_{2}\right) ; 114.1\left(2 \mathrm{CH}_{2}\right) ; 142.4(\mathrm{CH}) ; 142.7(\mathrm{C}) ; 143.1$ (C). IR (film): $3075,1640,890,785 \mathrm{~cm}^{-1}$. HRMS calcd for $\mathrm{C}_{24} \mathrm{H}_{42} \mathrm{NO}_{2}[\mathrm{M}+\mathrm{H}]^{+}$376.3210, found 376.3209.

(2S,4aS,7R,8aR)-3-(but-3-en-1-yl)-2-(3-methoxypenta1,4-dien-3-yl)-4,4,7-trimethyloctahydro-2H-

benzo $[e][1,3]$ oxazine (20d). This compound was obtained from 19d $(3.0 \mathrm{~g})$ and purified by flash chromatography (hexanes/ethyl acetate 50:1). Yield: $2.91 \mathrm{~g}, 93 \%$. Colorless oil. $[\alpha]_{\mathrm{D}}{ }^{25}=-69.6\left(c=1.4 \mathrm{CH}_{2} \mathrm{Cl}_{2}\right)$. $\mathrm{H}$ NMR $(300 \mathrm{MHz}$, $\left.\mathrm{CDCl}_{3}\right): \delta 0.88(\mathrm{~m}, 2 \mathrm{H}) ; 0.90(\mathrm{~d}, J=6.5 \mathrm{~Hz}, 3 \mathrm{H}) ; 1.03(\mathrm{~m}$, $1 \mathrm{H}) ; 1.13\left(\mathrm{~s}, 3 \mathrm{H}, \mathrm{CH}_{3}\right) ; 1.16\left(\mathrm{~s}, 3 \mathrm{H}, \mathrm{CH}_{3}\right) ; 1.39-1.47(\mathrm{~m}$, $2 \mathrm{H}) ; 1.32(\mathrm{~m}, 1 \mathrm{H}) ; 1.67(\mathrm{~m}, 1 \mathrm{H}) ; 1.91(\mathrm{~m}, 1 \mathrm{H}) ; 2.12(\mathrm{~m}$, $1 \mathrm{H}) ; 2.29(\mathrm{~m}, 1 \mathrm{H}) ; 2.55(\mathrm{~m}, 1 \mathrm{H}) ; 3.12(\mathrm{~m}, 1 \mathrm{H}) ; 3.23(\mathrm{~s}$, $3 \mathrm{H}) ; 3.44\left(\mathrm{td}, J_{l}=10.6 \mathrm{~Hz}, J_{2}=3.8 \mathrm{~Hz}, 1 \mathrm{H}\right) ; 4.57(\mathrm{~s}, 1 \mathrm{H})$; $4.91\left(\mathrm{dd}, J_{l}=9.9 \mathrm{HZ}, J_{2}=2.0 \mathrm{~Hz}, 1 \mathrm{H}\right) ; 4.96(\mathrm{~m}, 1 \mathrm{H}) ; 5.22-$ $5.35(\mathrm{~m}, 4 \mathrm{H}) ; 5.66(\mathrm{~m}, 1 \mathrm{H}) ; 5.96\left(\mathrm{dd}, J_{l}=17.6 \mathrm{~Hz}, J_{2}=\right.$ $9.9 \mathrm{~Hz}, 1 \mathrm{H}) ; 6.05\left(\mathrm{dd}, J_{l}=17.6 \mathrm{~Hz}, J_{2}=9.9 \mathrm{~Hz}, 1 \mathrm{H}\right)$. ). ${ }^{13} \mathrm{C}$ NMR (75 MHz, CDCl $\left.\mathrm{CDC}_{3}\right): \delta 22.2\left(\mathrm{CH}_{3}\right) ; 22.5\left(\mathrm{CH}_{3}\right)$; $25.1\left(\mathrm{CH}_{2}\right) ; 26.2\left(\mathrm{CH}_{3}\right) ; 31.4(\mathrm{CH}) ; 35.1\left(\mathrm{CH}_{2}\right) ; 37.3$ $\left(\mathrm{CH}_{2}\right) ; 41.4\left(\mathrm{CH}_{2}\right) ; 43.0\left(\mathrm{CH}_{2}\right) ; 44.9(\mathrm{CH}) ; 51.4\left(\mathrm{CH}_{3}\right)$; $57.6(\mathrm{C}) ; 77.5(\mathrm{CH}) ; 81.5(\mathrm{C}) ; 90.8(\mathrm{CH}) ; 114.7\left(\mathrm{CH}_{2}\right)$; $115.0\left(\mathrm{CH}_{2}\right) ; 116.9\left(\mathrm{CH}_{2}\right) ; 136.9(\mathrm{CH}) ; 137.2(\mathrm{CH}) ; 140.0$ (CH). IR (film): $3075,1640,1455,915 \mathrm{~cm}^{-1}$. HRMS calcd for $\mathrm{C}_{21} \mathrm{H}_{36} \mathrm{NO}_{2}[\mathrm{M}+\mathrm{H}]^{+}$334.2741, found 334.2747.
(2S,4aS,7R,8aR)-3-(but-3-en-1-yl)-2-(4-methoxy-2,6dimethylhepta-1,6-dien-4-yl)-4,4,7-trimethyloctahydro$\mathbf{2 H}$-benzo[e][1,3]oxazine (20e). This compound was obtained from 19e $(2.1 \mathrm{~g})$ and purified by flash chromatography (hexanes/ethyl acetate 80:1). Yield: $2.12 \mathrm{~g}$, 97\%. Colorless oil. $[\alpha]_{\mathrm{D}}{ }^{25}=-52.6\left(c=1.2 \mathrm{CH}_{2} \mathrm{Cl}_{2}\right)$. ${ }^{1} \mathrm{H}$ NMR $\left(300 \mathrm{MHz}, \mathrm{CDCl}_{3}\right): \delta 0.84-0.91(\mathrm{~m}, 2 \mathrm{H}) ; 0.90(\mathrm{~d}, J=$ $6.5 \mathrm{~Hz}, 3 \mathrm{H}) ; 0.98(\mathrm{~m}, 1 \mathrm{H}) ; 1.09(\mathrm{~s}, 3 \mathrm{H}) ; 1.14(\mathrm{~s}, 3 \mathrm{H}) ; 1.17-$ $1.79(\mathrm{~m}, 4 \mathrm{H}) ; 1.81(\mathrm{~s}, 3 \mathrm{H}) ; 1.82(\mathrm{~s}, 3 \mathrm{H}) ; 1.83(\mathrm{~m}, 1 \mathrm{H})$ $2.16(\mathrm{~d}, J=14.4 \mathrm{~Hz}, 1 \mathrm{H}) ; 2.25(\mathrm{~m}, 1 \mathrm{H}) ; 2.44(\mathrm{~d}, J=14.4$ $\mathrm{Hz}, 1 \mathrm{H}) ; 2.48-2.62(\mathrm{~m}, 2 \mathrm{H}) ; 2.63(\mathrm{~d}, J=14.4 \mathrm{~Hz}, 1 \mathrm{H})$; $2.69(\mathrm{~d}, J=14.4 \mathrm{~Hz}, 1 \mathrm{H}) ; 3.26\left(\mathrm{td}, J_{l}=10.3 \mathrm{~Hz}, J_{2}=4.0\right.$ $\mathrm{Hz}, 1 \mathrm{H}) ; 3.33(\mathrm{~m}, 1 \mathrm{H}) ; 3.41(\mathrm{~s}, 3 \mathrm{H}) ; 4.33(\mathrm{~s}, 1 \mathrm{H}) ; 4.84-$ $5.16(\mathrm{~m}, 6 \mathrm{H}) ; 5.53(\mathrm{~m}, 1 \mathrm{H}){ }^{13} \mathrm{C} \mathrm{NMR}\left(75 \mathrm{MHz}, \mathrm{CDCl}_{3}\right): \delta$ $22.3\left(\mathrm{CH}_{3}\right) ; 22.6\left(\mathrm{CH}_{3}\right) ; 25.1\left(2 \mathrm{CH}_{3}\right) ; 25.3\left(\mathrm{CH}_{2}\right) ; 26.5$ $\left(\mathrm{CH}_{3}\right) ; 31.5(\mathrm{CH}) ; 35.2\left(\mathrm{CH}_{2}\right) ; 37.9\left(\mathrm{CH}_{2}\right) ; 38.2\left(\mathrm{CH}_{2}\right)$ $41.5\left(\mathrm{CH}_{2}\right) ; 41.8\left(\mathrm{CH}_{2}\right) ; 43.2\left(\mathrm{CH}_{2}\right) ; 44.5(\mathrm{CH}) ; 51.5\left(\mathrm{CH}_{3}\right)$; $58.2(\mathrm{C}) ; 76.9(\mathrm{CH}) ; 81.4(\overline{\mathrm{C}}) ; 89.7(\mathrm{CH}) ; 114.1\left(\mathrm{CH}_{2}\right)$ $114.4\left(\mathrm{CH}_{2}\right) ; 114.6\left(\mathrm{CH}_{2}\right) ; 137.6(\mathrm{CH}) ; 142.8(\mathrm{C}) ; 143.4$ (C). IR (film): $3075,1640,750 \mathrm{~cm}^{-1}$. HRMS calcd for $\mathrm{C}_{25} \mathrm{H}_{44} \mathrm{NO}_{2}[\mathrm{M}+\mathrm{H}]^{+} 390.3367$, found 390.3376 .

General procedure for silylation of alcohols 19a-e. To a solution of alcohols 19a-e $(4.5 \mathrm{mmol})$ and 2,6 -lutidine $(1.1$ $\mathrm{mL}, 9.0 \mathrm{mmol})$ in anhydrous $\mathrm{CH}_{2} \mathrm{Cl}_{2}(25 \mathrm{~mL})$ was dropwise added tert-butyldimethylsilyl-triflate $(1.7 \mathrm{~mL}$, $7.2 \mathrm{mmol}$ ). The solution was stirred at room temperature until the starting material was fully consumed as indicated by TLC (for total transformation of alcohols 19b, 19c and 19e it was necessary to add an extra amount of 2,6 lutidine, $0.3 \mathrm{~mL}$, and TBSOTf, $0.45 \mathrm{~mL})$. The reaction liquid was diluted with $\mathrm{CH}_{2} \mathrm{Cl}_{2}(60 \mathrm{~mL})$, then washed with water, aqueous saturated ammonium chloride solution and aqueous saturated sodium chloride solution, then dried over anhydrous magnesium sulphate and concentrated. The residue was purified through silica gel column chromatography using hexanes/ ethyl acetate as an eluent.

\section{$(2 S, 4 a S, 7 R, 8 \mathrm{a} R)-3$-allyl-2-(3-( tert-}

butyldimethylsilyl)oxy)penta-1,4-dien-3-yl)-4,4,7-

trimethyloctahydro-2H-benzo[e][1,3]oxazine (21a). This compound was obtained from 19a $(1.4 \mathrm{~g})$ and purified by flash chromatography (hexanes/ethyl acetate 100:1). Yield: $1.85 \mathrm{~g}, 96 \%$. Colorless oil. $[\alpha]_{\mathrm{D}}{ }^{25}=-34.5\left(c=1.0 \mathrm{CH}_{2} \mathrm{Cl}_{2}\right)$. ${ }^{1} \mathrm{H}$ NMR $\left(300 \mathrm{MHz}, \mathrm{CDCl}_{3}\right): \delta 0.08(\mathrm{~s}, 3 \mathrm{H}) ; 0.10(\mathrm{~s}, 3 \mathrm{H})$ $0.85-90(\mathrm{~m}, 2 \mathrm{H}) ; 0.89(\mathrm{~s}, 9 \mathrm{H}) ; 0.93(\mathrm{~d}, J=6.5 \mathrm{~Hz}, 3 \mathrm{H})$ $1.04(\mathrm{~s}, 3 \mathrm{H}) ; 1.08(\mathrm{~m}, 1 \mathrm{H}) ; 1.14(\mathrm{~s}, 3 \mathrm{H}) ; 1.42-1.58(\mathrm{~m}$, $3 \mathrm{H}) ; 1.69(\mathrm{~m}, 1 \mathrm{H}) ; 1.90(\mathrm{~m}, 1 \mathrm{H}) ; 3.25\left(\mathrm{dd}, J_{l}=17.7 \mathrm{~Hz}\right.$ $\left.J_{2}=6.0 \mathrm{~Hz}, 1 \mathrm{H}\right) ; 3.43\left(\mathrm{td}, J_{1}=10.6 \mathrm{~Hz}, J_{2}=3.9 \mathrm{~Hz}, 1 \mathrm{H}\right)$ $3.88\left(\mathrm{dd}, J_{l}=17.7 \mathrm{~Hz}, J_{2}=5.0 \mathrm{~Hz}, 1 \mathrm{H}\right) ; 4.45(\mathrm{~s}, 1 \mathrm{H}) ; 4.85$ $\left(\mathrm{dd}, J_{l}=10.6 \mathrm{~Hz}, J_{2}=1.8 \mathrm{~Hz}, 1 \mathrm{H}\right) ; 4.93\left(\mathrm{dd}, J_{l}=17.4 \mathrm{~Hz}\right.$ $\left.J_{2}=1.8 \mathrm{~Hz}, 1 \mathrm{H}\right) ; 5.11\left(\mathrm{dd}, J_{1}=10.6 \mathrm{~Hz}, J_{2}=1.8 \mathrm{~Hz}, 1 \mathrm{H}\right)$ $5.16\left(\mathrm{dd}, J_{l}=10.6 \mathrm{~Hz}, J_{2}=1.8 \mathrm{~Hz}, 1 \mathrm{H}\right) ; 5.28\left(\mathrm{dd}, J_{l}=17.4\right.$ $\left.\mathrm{Hz}, J_{2}=1.8 \mathrm{~Hz}, 1 \mathrm{H}\right) ; 5.29\left(\mathrm{dd}, J_{l}=17.4 \mathrm{~Hz}, J_{2}=1.8 \mathrm{~Hz}\right.$ $1 \mathrm{H}) ; 5.89(\mathrm{~m}, 1 \mathrm{H}) ; 6.06\left(\mathrm{dd}, J_{l}=17.4 \mathrm{~Hz}, J_{2}=10.6 \mathrm{~Hz}\right.$ $1 \mathrm{H}) ; 6.12\left(\mathrm{dd}, J_{l}=17.4 \mathrm{~Hz}, J_{2}=10.6 \mathrm{~Hz}, 1 \mathrm{H}\right) .{ }^{13} \mathrm{C} \mathrm{NMR}$ $\left(75 \mathrm{MHz}, \mathrm{CDCl}_{3}\right): \delta-1.9\left(2 \mathrm{CH}_{3}\right) ; 18.7(\mathrm{C}) ; 22.4\left(\mathrm{CH}_{3}\right)$ $22.8\left(\mathrm{CH}_{3}\right) ; 25.0\left(\mathrm{CH}_{2}\right) ; 26.1\left(3 \mathrm{CH}_{3}\right) ; 26.9\left(\mathrm{CH}_{3}\right) ; 31.5$ $(\mathrm{CH}) ; 35.2\left(\mathrm{CH}_{2}\right) ; 41.4\left(\mathrm{CH}_{2}\right) ; 41.5(\mathrm{CH}) ; 46.6\left(\mathrm{CH}_{2}\right) ; 57.9$ (C); $77.3(\mathrm{CH}) ; 80.0(\mathrm{C}) ; 91.1(\mathrm{CH}) ; 112.2\left(\mathrm{CH}_{2}\right) ; 114.2$ $\left(\mathrm{CH}_{2}\right) ; 114.5\left(\mathrm{CH}_{2}\right) ; 139.7(\mathrm{CH}) ; 141.3(\mathrm{CH}) ; 142.8(\mathrm{CH})$ IR (film): 3080, 1635, 835, 780, $685 \mathrm{~cm}^{-1}$. HRMS calcd for $\mathrm{C}_{25} \mathrm{H}_{46} \mathrm{NO}_{2} \mathrm{Si}[\mathrm{M}+\mathrm{H}]^{+} 420.3292$, found 420.3292 .

\section{$(2 S, 4 a S, 7 R, 8 \mathrm{a} R)-3$-allyl-2-(3-( tert-}

butyldimethylsilyl)oxy)-2,4-dimethylpenta-1,4-dien-3yl)-4,4,7-trimethyloctahydro- $2 H$-benzo $[e][1,3]$ oxazine (21b). This compound was obtained from 19b $(1.65 \mathrm{~g})$ and purified by flash chromatography (hexanes $/ \mathrm{CH}_{2} \mathrm{Cl}_{2} 3: 1$ ). Yield: $1.33 \mathrm{~g}$ 60\%. White solid. Mp 53-54 ${ }^{\circ} \mathrm{C}$ (from hexane $) .[\alpha]^{25}=-2.0\left(c=1.0 \mathrm{CH}_{2} \mathrm{Cl}_{2}\right) .{ }^{1} \mathrm{H}$ NMR $(300$ $\left.\mathrm{MHz}, \mathrm{CDCl}_{3}\right): \delta 0.07(\mathrm{~s}, 3 \mathrm{H}) ; 0.23(\mathrm{~s}, 3 \mathrm{H}) ; 0.87-1.05(\mathrm{~m}$, $2 \mathrm{H}) ; 0.91\left(\mathrm{~s}, 9 \mathrm{H}, 3 \mathrm{CH}_{3}\right) ; 0.94(\mathrm{~d}, 3 \mathrm{H}, J=6.5 \mathrm{~Hz}) ; 1.02(\mathrm{~s}$ $3 \mathrm{H}) ; 1.16(\mathrm{~m}, 1 \mathrm{H}) ; 1.20(\mathrm{~s}, 3 \mathrm{H}) ; 1.38-1.60(\mathrm{~m}, 3 \mathrm{H}) ; 1.64$ $(\mathrm{s}, 6 \mathrm{H}) ; 1.73(\mathrm{~m}, 1 \mathrm{H}) ; 1.87(\mathrm{~m}, 1 \mathrm{H}) ; 3.12\left(\mathrm{dd}, J_{l}=17.3 \mathrm{~Hz}\right.$, $\left.J_{2}=7.6 \mathrm{~Hz}, 1 \mathrm{H}\right) ; 3.51\left(\mathrm{td}, J_{1}=10.6 \mathrm{~Hz}, J_{2}=3.8 \mathrm{~Hz}, 1 \mathrm{H}\right)$; $4.19(\mathrm{~m}, 1 \mathrm{H}) ; 4.71(\mathrm{~m}, 1 \mathrm{H}) ; 4.82-4.88(\mathrm{~m}, 2 \mathrm{H}) ; 4.97(\mathrm{~s}$ $1 \mathrm{H}) ; 4.98-5.00(\mathrm{~m}, 2 \mathrm{H}) ; 5.30(\mathrm{~m}, 1 \mathrm{H}) ; 5.77(\mathrm{~m}, 1 \mathrm{H}) .{ }^{13} \mathrm{C}$ NMR $\left(75 \mathrm{MHz}, \mathrm{CDCl}_{3}\right): \delta-3.7\left(\mathrm{CH}_{3}\right) ;-1.3\left(\mathrm{CH}_{3}\right) ; 18.1$ (C); $19.4\left(\mathrm{CH}_{3}\right) ; 19.9\left(\mathrm{CH}_{3}\right) ; 22.4\left(\mathrm{CH}_{3}\right) ; 22.5\left(\mathrm{CH}_{3}\right) ; 25.1$ 
$\left(\mathrm{CH}_{2}\right) ; 26.8\left(3 \mathrm{CH}_{3}\right) ; 27.9\left(\mathrm{CH}_{3}\right) ; 31.7(\mathrm{CH}) ; 35.3\left(\mathrm{CH}_{2}\right)$; $41.4\left(\mathrm{CH}_{2}\right) ; 43.8(\mathrm{CH}) ; 47.5\left(\mathrm{CH}_{2}\right) ; 58.4(\mathrm{C}) ; 76.9(\mathrm{CH})$; $84.9(\mathrm{CH}) ; 85.5(\mathrm{C}) ; 110.2\left(\mathrm{CH}_{2}\right) ; 112.1\left(\mathrm{CH}_{2}\right) ; 113.7$ $\left(\mathrm{CH}_{2}\right) ; 144.5(\mathrm{CH}) ; 144.7(\mathrm{C}) ; 146.4(\mathrm{C})$. IR' (nujol dispersion): $3070,1638,835,687 \mathrm{~cm}^{-1}$. HRMS calcd for $\mathrm{C}_{27} \mathrm{H}_{50} \mathrm{NO}_{2} \mathrm{Si}[\mathrm{M}+\mathrm{H}]^{+} 448.3605$, found 448.3617 .

\section{$(2 S, 4 \mathrm{a} S, 7 R, 8 \mathrm{a} R)-3$-allyl-2-(4-( (tert-}

butyldimethylsilyl)oxy)-2,6-dimethylhepta-1,6-dien-4yl)-4,4,7-trimethyloctahydro- $2 H$-benzo $[e][1,3]$ oxazine (21c). This compound was obtained from 19c $(1.7 \mathrm{~g})$ and purified by flash chromatography (hexanes/ ethyl acetate 100:1). Yield: $1.75 \mathrm{~g}, 78 \%$. Colorless oil. $[\alpha]_{\mathrm{D}}{ }^{25}=-31.0(c$ $\left.=1.5 \mathrm{CH}_{2} \mathrm{Cl}_{2}\right) \cdot{ }^{1} \mathrm{H}$ NMR $\left(300 \mathrm{MHz}, \mathrm{CDCl}_{3}\right): \delta 0.12(\mathrm{~s}$, $3 \mathrm{H}) ; 0.22(\mathrm{~s}, 3 \mathrm{H}) ; 0.86-1.04(\mathrm{~m}, 2 \mathrm{H}) ; 0.89$ (s, 9H); 0.94 (d, $J=6.5 \mathrm{~Hz}, 3 \mathrm{H}) ; 1.05(\mathrm{~s}, 3 \mathrm{H}) ; 1.09(\mathrm{~m}, 1 \mathrm{H}) ; 1.12(\mathrm{~s}, 3 \mathrm{H})$; $1.45-1.58(\mathrm{~m}, 3 \mathrm{H}) ; 1.70(\mathrm{~m}, 1 \mathrm{H}) ; 1.83(\mathrm{~s}, 6 \mathrm{H}) ; 1.88(\mathrm{~m}$, $1 \mathrm{H}) ; 2.16(\mathrm{~d}, J=14.5 \mathrm{~Hz}, 1 \mathrm{H}) ; 2.52(\mathrm{~d}, J=13.8 \mathrm{~Hz}, 1 \mathrm{H})$; $2.66(\mathrm{~d}, 1 \mathrm{H}, J=13.8 \mathrm{~Hz}) ; 2.68(\mathrm{~d}, J=14.5 \mathrm{~Hz}, 1 \mathrm{H}) ; 3.25$ $\left(\mathrm{dd}, J_{l}=18.2 \mathrm{~Hz}, J_{2}=5.7 \mathrm{~Hz}, 1 \mathrm{H}\right) ; 3.39\left(\mathrm{td}, J_{l}=10.6 \mathrm{~Hz}\right.$, $\left.J_{2}=3.8 \mathrm{~Hz}, 1 \mathrm{H}\right) ; 4.41(\mathrm{~s}, 1 \mathrm{H}) ; 4.42\left(\mathrm{dd}, J_{1}=18.2 \mathrm{~Hz}, J_{2}=\right.$ $4.2 \mathrm{~Hz}, 1 \mathrm{H}) ; 4.85-4.96(\mathrm{~m}, 5 \mathrm{H}) ; 5.11\left(\mathrm{dd}, J_{l}=17.4 \mathrm{~Hz}, J_{2}\right.$ $=1.8 \mathrm{~Hz}, 1 \mathrm{H}) ; 5.98(\mathrm{~m}, 1 \mathrm{H}){ }^{13} \mathrm{C}$ NMR $\left(75 \mathrm{MHz} \mathrm{CDCl}_{3}\right)$ : $\delta-0.9\left(2 \mathrm{CH}_{3}\right) ; 19.1(\mathrm{C}) ; 22.4\left(\mathrm{CH}_{3}\right) ; 22.9\left(\mathrm{CH}_{3}\right) ; 25.0$ $\left(\mathrm{CH}_{2}\right) ; 25.1\left(\mathrm{CH}_{3}\right) ; 26.2\left(\mathrm{CH}_{3}\right) ; 26.6\left(3 \mathrm{CH}_{3}\right) ; 27.1\left(\mathrm{CH}_{3}\right)$; $31.5(\mathrm{CH}) ; 35.3\left(\mathrm{CH}_{2}\right) ; 41.3\left(\mathrm{CH}_{2}\right) ; 42.1\left(\mathrm{CH}_{2}\right) ; 43.7(\mathrm{CH})$; $45.0\left(\mathrm{CH}_{2}\right) ; 46.4\left(\mathrm{CH}_{2}\right) ; 58.2(\mathrm{C}) ; 76.5(\mathrm{CH}) ; 81.9(\mathrm{C})$; $86.9(\mathrm{CH}) ; 112.4\left(\mathrm{CH}_{2}\right) ; 114.6\left(\mathrm{CH}_{2}\right) ; 115.0\left(\mathrm{CH}_{2}\right) ; 142.7$ (CH); 142.9 (2C). IR (film): 3075, 1640, 775, 700, $670 \mathrm{~cm}$ . HRMS calcd for $\mathrm{C}_{29} \mathrm{H}_{54} \mathrm{NO}_{2} \mathrm{Si}[\mathrm{M}+\mathrm{H}]^{+} 476.3918$, found 476.3917

$(2 S, 4 a S, 7 R, 8 a R)-3-(b u t-3-e n-1-y l)-2-(3-((t e r t-$ butyldimethylsilyl)oxy)penta-1,4-dien-3-yl)-4,4,7trimethyloctahydro-2 $H$-benzo $[e][1,3]$ oxazine $(21 d)$. This compound was obtained from 19d $(1.0 \mathrm{~g})$ and purified by flash chromatography (hexanes/ ethyl acetate 100:1). Yield: $1.33 \mathrm{~g}, 98 \%$. Colorless oil. $[\alpha]_{\mathrm{D}}{ }^{25}=-27.1(c=1.0$ $\mathrm{CH}_{2} \mathrm{Cl}_{2}$ ). ${ }^{1} \mathrm{H}$ NMR (400 MHz, $\left.\mathrm{CDCl}_{3}\right): \delta 0.09$ (s, 6H), $0.84-0.95(\mathrm{~m} 2 \mathrm{H}), 0.89(\mathrm{~s}, 9 \mathrm{H}), 0.90(\mathrm{~d}, 3 \mathrm{H}, J=6.5 \mathrm{~Hz})$, $1.07(\mathrm{~m}, 1 \mathrm{H}), 1.09(\mathrm{~s}, 3 \mathrm{H}), 1.13(\mathrm{~s}, 3 \mathrm{H}), 1.39-1.48(\mathrm{~m}, 2 \mathrm{H})$, $1.55(\mathrm{~m}, 1 \mathrm{H}), 1.67(\mathrm{~m}, 1 \mathrm{H}), 1.88(\mathrm{~m}, 1 \mathrm{H}), 2.06(\mathrm{~m}, 1 \mathrm{H})$, $2.23(\mathrm{~m}, 1 \mathrm{H}), 2.46(\mathrm{~m}, 1 \mathrm{H}), 3.19(\mathrm{~m}, 1 \mathrm{H}), 3.41\left(\mathrm{td}, J_{1}=\right.$ $\left.10.4 \mathrm{~Hz}, J_{2}=3.8 \mathrm{~Hz}, 1 \mathrm{H}\right), 4.43(\mathrm{~s}, 1 \mathrm{H}), 4.84-4.93(\mathrm{~m}, 2 \mathrm{H})$, $5.10(\mathrm{~d}, J=10.6 \mathrm{~Hz}, 1 \mathrm{H}), 5.15$ (d, $J=10.6 \mathrm{~Hz}, 1 \mathrm{H}), 5.25-$ $5.35(\mathrm{~m}, 2 \mathrm{H}), 5.62(\mathrm{~m}, 1 \mathrm{H}), 6.02\left(\mathrm{dd}, J_{1}=17.3 \mathrm{~Hz}, J_{2}=\right.$ $10.6 \mathrm{~Hz}, 1 \mathrm{H}), 6.14\left(\mathrm{dd}, J_{1}=17.3 \mathrm{~Hz}, J_{2}=10.6 \mathrm{~Hz}, 1 \mathrm{H}\right)$. ${ }^{13} \mathrm{C}$ NMR $\left(75 \mathrm{MHz}, \mathrm{CDCl}_{3}\right): \delta-1.8\left(2 \mathrm{CH}_{3}\right) ; 18.3(\mathrm{C}) ; 22.4$ $\left(\mathrm{CH}_{3}\right) ; 22.9\left(\mathrm{CH}_{3}\right) ; 25.2\left(\mathrm{CH}_{2}\right) ; 26.1\left(3 \mathrm{CH}_{3}\right) ; 26.4\left(\mathrm{CH}_{3}\right)$; $31.5(\mathrm{CH}) ; 35.3\left(\mathrm{CH}_{2}\right) ; 37.8\left(\mathrm{CH}_{2}\right) ; 41.4\left(\mathrm{CH}_{2}\right) ; 43.0$ $\left(\mathrm{CH}_{2}\right) ; 44.4(\mathrm{CH}) ; 57.5(\mathrm{C}) ; 77.4(\mathrm{CH}) ; 79.9(\mathrm{C}) ; 91.1$ $(\mathrm{CH}) ; 114.0\left(\mathrm{CH}_{2}\right) ; 114.2\left(\mathrm{CH}_{2}\right) ; 114.7\left(\mathrm{CH}_{2}\right) ; 137.0(\mathrm{CH})$; 140.4 (CH); 141.3 (CH). IR (film): 3080, 2925, 1640, 1470, $1460,835,780,685 \mathrm{~cm}^{-1}$. HRMS calcd for $\mathrm{C}_{26} \mathrm{H}_{48} \mathrm{NO}_{2} \mathrm{Si}$ $[\mathrm{M}+\mathrm{H}]^{+}$434.3449, found 434.3456.

\section{(2S,4aS,7R,8aR)-3-(but-3-en-1-yl)-2-(4-(tert-} butyldimethylsilyl)oxy)-2,6-dimethylhepta-1,6-dien-4yl)-4,4,7-trimethyloctahydro- $2 H$-benzo $[e][1,3]$ oxazine (21e). This compound was obtained from 19e (1.14 g) and purified by flash chromatography (hexanes/ ethyl acetate 100:1). Yield: $1.23 \mathrm{~g}, 83 \%$. Colorless oil. $[\alpha]_{\mathrm{D}}{ }^{25}=-35.3(c$ $\left.=1.5 \mathrm{CH}_{2} \mathrm{Cl}_{2}\right) \cdot{ }_{\mathrm{H}} \mathrm{NMR}\left(300 \mathrm{MHz}, \mathrm{CDCl}_{3}\right): \delta 0.12(\mathrm{~s}$, $3 \mathrm{H}) ; 0.19(\mathrm{~s}, 3 \mathrm{H}) ; 89-1.04(\mathrm{~m}, 3 \mathrm{H}) ; 0.91(\mathrm{~s}, 9 \mathrm{H}) ; 0.93(\mathrm{~d}$, $3 \mathrm{H}, J=6.6 \mathrm{~Hz}) ; 1.08(\mathrm{~m}, 1 \mathrm{H}) ; 1.10(\mathrm{~s}, 3 \mathrm{H}) ; 1.12(\mathrm{~s}, 3 \mathrm{H})$; $1.32-1.62(\mathrm{~m}, 3 \mathrm{H}) ; 1.71(\mathrm{~m}, 1 \mathrm{H}) ; 1.82(\mathrm{~s}, 3 \mathrm{H}) ; 1.84(\mathrm{~m}$, $1 \mathrm{H}) ; 1.89$ (s, 3H); 2.17 (d, $1 \mathrm{H}, J=14.6 \mathrm{~Hz}) ; 2.28(\mathrm{~m}, 1 \mathrm{H})$; $2.38-2.50(\mathrm{~m}, 3 \mathrm{H}) ;-2.61-2.72(\mathrm{~m}, 2 \mathrm{H}), 3.38\left(\mathrm{td}, 1 \mathrm{H}, J_{1}=\right.$ $\left.10.6 \mathrm{~Hz}, J_{2}=3.6 \mathrm{~Hz}\right) ; 3.68(\mathrm{~m}, 1 \mathrm{H}) ; 3.36(\mathrm{~s}, 1 \mathrm{H}) ; 4.85-$ $5.04(\mathrm{~m}, 6 \mathrm{H}) ; 5.78(\mathrm{~m}, 1 \mathrm{H}) .{ }^{13} \mathrm{C}$ NMR $\left(75 \mathrm{MHz}, \mathrm{CDCl}_{3}\right): \delta$ $-0.91\left(2 \mathrm{CH}_{3}\right) ; 19.2(\mathrm{C}) ; 22.3\left(\mathrm{CH}_{3}\right) ; 23.0\left(\mathrm{CH}_{3}\right) ; 25.0$ $\left(\mathrm{CH}_{2}\right) ; 25.1\left(\mathrm{CH}_{3}\right) ; 26.3\left(\mathrm{CH}_{3}\right) ; 26.6\left(3 \mathrm{CH}_{3}\right) ; 26.8\left(\mathrm{CH}_{3}\right) ;$ $31.5(\mathrm{CH}) ; 35.2\left(\mathrm{CH}_{2}\right) ; 38.3\left(\mathrm{CH}_{2}\right) ; 41.2\left(\mathrm{CH}_{2}\right) ; 42.4$ $\left(\mathrm{CH}_{2}\right) ; 43.1\left(\mathrm{CH}_{2}\right) ; 43.5(\mathrm{CH}) ; 45.0\left(\mathrm{CH}_{2}\right) ; 58.1(\mathrm{C}) ; 76.5$ $(\mathrm{CH}) ; 81.8(\mathrm{C}) ; 87.1(\mathrm{CH}) ; 114.8\left(2 \mathrm{CH}_{2}\right) ; 115.0\left(\mathrm{CH}_{2}\right)$; $137.2(\mathrm{CH}) ; 142.9$ (C); 143.0 (C). IR (film): 3080, 2950, $1640,1460,835,775 \mathrm{~cm}^{-1}$. HRMS calcd for $\mathrm{C}_{30} \mathrm{H}_{56} \mathrm{NO}_{2} \mathrm{Si}$ $[\mathrm{M}+\mathrm{H}]^{+} 490.4075$, found 490.4078 .
General procedure for DSRCM Reaction of trienes 4ag, 19a-e, 20a-e, 21a-e and diene 14. The triene $(3 \mathrm{mmol})$ was dissolved in dry $\mathrm{CH}_{2} \mathrm{Cl}_{2}(90 \mathrm{~mL})$ and argon was bubbled. Grubbs first- or second-generation catalyst $(0.06$ mmol) was added and the resulting mixture was stirred at room temperature or at reflux under argon. The reaction progress was monitored by TLC and additional portions of fresh catalyst were added after the times indicated in Tables 1 and 2 . When the reaction was found to be complete the solvent was evaporated in vacuo and the residue was purified by flash chromatography on silica gel using mixtures of hexanes/ethyl acetate as eluent.

\section{$(2 S, 4 a S, 7 R, 8 a R)-3-b e n z y l-4,4,7-t r i m e t h y l-2-((R)-2-$ vinyl-2,5-dihydrofuran-2-yl)octahydro- $2 \mathrm{H}$ -}

benzo[ $[\boldsymbol{e}][1,3]$ oxazine $(7 \mathbf{a})$. This compound was obtained from $\mathbf{4 a}(1.2 \mathrm{~g})$ and catalyst $\mathbf{5}$, and purified by flash chromatography (hexanes/ ethyl acetate 45:1). Yield: 0.62 g, 56\%. Colorless oil. $[\alpha]_{\mathrm{D}}{ }^{25}=-32.9\left(\mathrm{c}=1.8, \mathrm{CHCl}_{3}\right) .{ }^{1} \mathrm{H}$ NMR $\left(300 \mathrm{MHz}, \mathrm{CDCl}_{3}\right): \delta 0.84-1.02(\mathrm{~m}, 2 \mathrm{H}), 0.87(\mathrm{~s}$, $3 \mathrm{H}) ; 0.93(\mathrm{~d}, J=6.5 \mathrm{~Hz}, 3 \mathrm{H}) ; 1.14(\mathrm{~m}, 1 \mathrm{H}) ; 1.27(\mathrm{~s}, 3 \mathrm{H})$ $1.34-1.50(\mathrm{~m}, 3 \mathrm{H}) ; 1.60(\mathrm{~m}, 1 \mathrm{H}) ; 1.94(\mathrm{~m}, 1 \mathrm{H}) ; 3.51\left(\mathrm{td}, J_{1}\right.$ $=10.5 \mathrm{~Hz}, J=3.9 \mathrm{~Hz}, 1 \mathrm{H}) ; 3.87(\mathrm{~d}, J=18.3 \mathrm{~Hz}, 1 \mathrm{H})$ $4.45-4.52(\mathrm{~m}, 2 \mathrm{H}) ; 4.58(\mathrm{~d}, J=18.3 \mathrm{~Hz}, 1 \mathrm{H}) ; 4.78(\mathrm{~s}, 1 \mathrm{H})$ $4.97\left(\mathrm{dd}, J_{1}=10.7 \mathrm{~Hz}, J_{2}=1.7 \mathrm{~Hz}, 1 \mathrm{H}\right) ; 5.02\left(\left(\mathrm{dd}, J_{1}=\right.\right.$ $\left.17.3 \mathrm{~Hz}, J_{2}=1.7 \mathrm{~Hz}, 1 \mathrm{H}\right) ; 5.73-5.81(\mathrm{~m}, 2 \mathrm{H}) ; 6.16\left(\mathrm{dd}, J_{1}\right.$ $\left.=17.3 \mathrm{~Hz}, J_{2}=10.7 \mathrm{~Hz}, 1 \mathrm{H}\right) ; 7.14(\mathrm{~m}, 1 \mathrm{H}) ; 7.21-7.30(\mathrm{~m}$ 2H); 7.36-7.71 (m, 2H). ${ }^{13} \mathrm{C}$ NMR $\left(75 \mathrm{MHz} \mathrm{CDCl}_{3}\right): \delta$ $22.3\left(\mathrm{CH}_{3}\right) ; 22.8\left(\mathrm{CH}_{3}\right) ; 25.0\left(\mathrm{CH}_{2}\right) ; 26.7\left(\mathrm{CH}_{3}\right) ; 31.4$ $(\mathrm{CH}) ; 35.2\left(\mathrm{CH}_{2}\right) ; 41.4\left(\mathrm{CH}_{2}\right) ; 44.3(\mathrm{CH}) ; 47.2\left(\mathrm{CH}_{2}\right) ; 57.9$ (C); $75.3\left(\mathrm{CH}_{2}\right) ; 77.4(\mathrm{CH}) ; 90.6(\mathrm{CH}) ; 93.5(\mathrm{C}) ; 113.3$ $\left(\mathrm{CH}_{2}\right) ; 125.2(\mathrm{CH}) ; 126.6(\mathrm{CH}) ; 127.0(2 \mathrm{CH}) ; 127.5(2$ $\mathrm{CH}) ; 130.3(\mathrm{CH}) ; 138.7(\mathrm{CH}) ; 144.4(\mathrm{C})$. IR (film): 3060 $1730,720,695 \mathrm{~cm}^{-1}$. HRMS calcd for $\mathrm{C}_{24} \mathrm{H}_{34} \mathrm{NO}_{2}[\mathrm{M}+$ $\mathrm{H}]^{+} 368.2584$, found 368.2586 .

$(2 S, 4 \mathrm{a} S, 7 R, 8 \mathrm{a} R)-3-b e n z y l-4,4,7-t$ rimethyl-2-( $(S)$-2-vinyl2,5-dihydrofuran-2-yl)octahydro- $2 H$ -

benzo $[\boldsymbol{e}][1,3]$ oxazine $(\mathbf{8 a})$. This compound was obtained from $4 \mathbf{a}(1.2 \mathrm{~g})$ and catalyst $\mathbf{6}$, and purified by flash chromatography (hexanes/ ethyl acetate 45:1). Yield: 0.57 g, $51 \%$. Colorless oil. $[\alpha]_{\mathrm{D}}{ }^{25}=+1.9(\mathrm{c}=0.5$, ethyl acetate $)$. ${ }^{9} \mathrm{H}$ NMR (300 MHz, $\left.\mathrm{CDCl}_{3}\right): \delta 0.86-1.03(\mathrm{~m}, 2 \mathrm{H}) ; 0.88(\mathrm{~s}$, $3 \mathrm{H}) ; 0.92(\mathrm{~d}, J=6.5 \mathrm{~Hz}, 3 \mathrm{H}) ; 1.18(\mathrm{~m}, 1 \mathrm{H}) ; 1.26(\mathrm{~s}, 3 \mathrm{H})$ $1.29-1.67(\mathrm{~m}, 4 \mathrm{H}) ; 1.93(\mathrm{~m}, 1 \mathrm{H}) ; 3.51\left(\mathrm{td}, J_{1}=10.7 \mathrm{~Hz}, J_{2}\right.$ $=3.5 \mathrm{~Hz}, 1 \mathrm{H}) ; 3.96(\mathrm{~d}, J=18.6 \mathrm{~Hz}, 1 \mathrm{H}) ; 4.37-4.53(\mathrm{~m}$, $2 \mathrm{H}) ; 4.42(\mathrm{~d}, J=18.6 \mathrm{~Hz}, 1 \mathrm{H}) ; 4.74(\mathrm{~s}, 1 \mathrm{H}) ; 4.99\left(\mathrm{dd}, J_{1}=\right.$ $\left.10.6 \mathrm{~Hz}, J_{2}=1.7 \mathrm{~Hz}, 1 \mathrm{H}\right) ; 5.16\left(\mathrm{dd}, J_{1}=17.3 \mathrm{~Hz}, J_{2}=1.7\right.$ $\mathrm{Hz}, 1 \mathrm{H}) ; 5.63(\mathrm{~m}, 1 \mathrm{H}) ; 5.79(\mathrm{~m}, 1 \mathrm{H}) ; 6.07\left(\mathrm{dd}, J_{1}=17.3\right.$ $\left.\mathrm{Hz}, J_{2}=10.6 \mathrm{~Hz}, 1 \mathrm{H}\right) ; 7.13(\mathrm{~m}, 1 \mathrm{H}) ; 7.20-7.25(\mathrm{~m}, 2 \mathrm{H})$

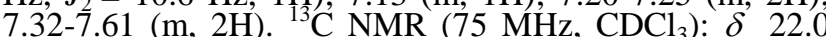
$\left(\mathrm{CH}_{3}\right) ; 22.2\left(\mathrm{CH}_{3}\right) ; 24.9\left(\mathrm{CH}_{2}\right) ; 26.6\left(\mathrm{CH}_{3}\right) ; 31.4(\mathrm{CH})$; $35.1\left(\mathrm{CH}_{2}\right) ; 41.4\left(\mathrm{CH}_{2}\right) ; 44.8(\mathrm{CH}) ; 46.9\left(\mathrm{CH}_{2}\right) ; 58.2(\mathrm{C})$ $74.8\left(\mathrm{CH}_{2}\right) ; 77.8(\mathrm{CH}) ; 90.5(\mathrm{CH}) ; 94.0(\mathrm{C}) ; 113.0\left(\mathrm{CH}_{2}\right)$ $125.2(\mathrm{CH}) ; 126.7(\mathrm{CH}) ; 127.0(2 \mathrm{CH}) ; 127.3(2 \mathrm{CH})$ $129.4(\mathrm{CH}) ; 139.9(\mathrm{CH}) ; 144.2(\mathrm{C})$. IR (film): 3060,1600 , $720,700 \mathrm{~cm}^{-1}$. HRMS calcd for $\mathrm{C}_{24} \mathrm{H}_{34} \mathrm{NO}_{2}[\mathrm{M}+\mathrm{H}]^{+}$ 368.2584 , found 368.2589 .

\section{$(2 S, 4 \mathrm{a} S, 7 R, 8 \mathrm{a} R)-3$-benzyl-4,4,7-trimethyl-2-( $(R)-4$ -} methyl-2-vinyl-2,5-dihydrofuran-2-yl)octahydro- $2 \mathrm{H}$ benzo $[e][1,3]$ oxazine $(7 \mathbf{b})$. This compound was obtained from $\mathbf{4 b}(1.2 \mathrm{~g})$ and catalyst $\mathbf{6}$, and purified by flash chromatography (hexanes/ ethyl acetate 50:1). Yield: 0.15 g, $13 \%$. Colorless oil. $[\alpha]_{\mathrm{D}}{ }^{25}=-20.1\left(\mathrm{c}=0.5, \mathrm{CH}_{2} \mathrm{Cl}_{2}\right) .{ }^{1} \mathrm{H}$ NMR $\left(300 \mathrm{MHz}, \mathrm{CDCl}_{3}\right): \delta \quad 0.82-1.06(\mathrm{~m}, 2 \mathrm{H}) ; 0.89(\mathrm{~s}$ $3 \mathrm{H}) ; 0.93(\mathrm{~d}, J=6.5 \mathrm{~Hz}, 3 \mathrm{H}) ; 1.12(\mathrm{~m}, 1 \mathrm{H}) ; 1.27(\mathrm{~s}, 3 \mathrm{H})$ $1.40-1.76(\mathrm{~m}, 5 \mathrm{H}) ; 1.59(\mathrm{~d}, J=1.1 \mathrm{~Hz}, 3 \mathrm{H}) ; 1.93(\mathrm{~m}, 1 \mathrm{H})$ $3.49\left(\mathrm{td}, J_{1}=10.4 \mathrm{~Hz}, J_{2}=3.8 \mathrm{~Hz}, 1 \mathrm{H}\right) ; 3.88(\mathrm{~d}, J=18.4 \mathrm{~Hz}$, $1 \mathrm{H}) ; 4.22-4.34(\mathrm{~m}, 2 \mathrm{H}) ; 4.61(\mathrm{~d}, J=18.4 \mathrm{~Hz}, 1 \mathrm{H}) ; 4.73(\mathrm{~s}$, $1 \mathrm{H}) ; 4.94\left(\mathrm{dd}, J_{1}=10.7 \mathrm{~Hz}, J_{2}=1.8 \mathrm{~Hz}, 1 \mathrm{H}\right) ; 5.03\left(\mathrm{dd}, J_{1}\right.$ $\left.=17.3 \mathrm{~Hz}, J_{2}=1.8 \mathrm{~Hz}, 1 \mathrm{H}\right) ; 5.36(\mathrm{~m}, 1 \mathrm{H}) ; 6.13\left(\mathrm{dd}, J_{1}=\right.$ $\left.17.3 \mathrm{~Hz}, J_{2}=10.7 \mathrm{~Hz}, 1 \mathrm{H}\right) ; 7.11(\mathrm{~m}, 1 \mathrm{H}) ; 7.17-7.26(\mathrm{~m}$, $2 \mathrm{H}) ; 7.30-7.43(\mathrm{~m}, 2 \mathrm{H}) .{ }^{13} \mathrm{C}$ NMR $\left(75 \mathrm{MHz} \mathrm{CDCl}_{3}\right): \delta$ $12.3\left(\mathrm{CH}_{3}\right) ; 22.3\left(\mathrm{CH}_{3}\right) ; 22.6\left(\mathrm{CH}_{3}\right) ; 25.0\left(\mathrm{CH}_{2}\right) ; 26.7$ $\left(\mathrm{CH}_{3}\right) ; 31.5(\mathrm{CH}) ; 35.2\left(\mathrm{CH}_{2}\right) ; 41.4\left(\mathrm{CH}_{2}\right) ; 44.5(\mathrm{CH}) ; 47.2$ $\left(\mathrm{CH}_{2}\right) ; 57.9(\mathrm{C}) ; 77.4(\mathrm{CH}) ; 77.7\left(\mathrm{CH}_{2}\right) ; 90.8(\mathrm{CH}) ; 93.9$ (C); $112.5\left(\mathrm{CH}_{2}\right) ; 124.0(\mathrm{CH}) ; 125.2(\mathrm{CH}) ; 127.1(2 \mathrm{CH})$; 127.5 (2 CH); 139.4 (C); $144.5(\mathrm{CH}) ; 158.7$ (C). IR (film): 
$3060,1450,720,695 \mathrm{~cm}^{-1}$. HRMS calcd for $\mathrm{C}_{25} \mathrm{H}_{36} \mathrm{NO}_{2}[\mathrm{M}$ $+\mathrm{H}]^{+}$382.2741, found 382.2750.

(2S,4aS,7R,8aR)-3-benzyl-4,4,7-trimethyl-2- $((S)-4$ methyl-2-vinyl-2,5-dihydrofuran-2-yl)octahydro- $2 \mathrm{H}$ benzo $[e][1,3]$ oxazine $(\mathbf{8 b})$. This compound was obtained from $\mathbf{4 b}(1.2 \mathrm{~g})$ and catalyst $\mathbf{6}$, and purified by flash chromatography (hexanes/ ethyl acetate 50:1). Yield: 0.19 g, $17 \%$. Colorless oil. $[\alpha]_{\mathrm{D}}{ }^{25}=-2.8\left(\mathrm{c}=0.5, \mathrm{CH}_{2} \mathrm{Cl}_{2}\right) .{ }^{1} \mathrm{H}$ NMR $\left(300 \mathrm{MHz}, \mathrm{CDCl}_{3}\right): \delta \quad 0.82-1.05(\mathrm{~m}, 2 \mathrm{H}) ; 0.89(\mathrm{~s}$, $3 \mathrm{H}) ; 0.92(\mathrm{~d}, J=6.5 \mathrm{~Hz}, 3 \mathrm{H}) ; 1.22(\mathrm{~m}, 1 \mathrm{H}) ; 1.27(\mathrm{~s}, 3 \mathrm{H})$; $1.28(\mathrm{~s}, 3 \mathrm{H}) ; 1.40-1.78(\mathrm{~m}, 4 \mathrm{H}) ; 1.93(\mathrm{~m}, 1 \mathrm{H}) ; 3.49\left(\mathrm{td}, J_{1}=\right.$ $\left.10.5 \mathrm{~Hz}, J_{2}=3.9 \mathrm{~Hz}, 1 \mathrm{H}\right) ; 3.92(\mathrm{~d}, J=18.7 \mathrm{~Hz}, 1 \mathrm{H}) ; 4.07$ $(\mathrm{d}, J=12.3 \mathrm{~Hz}, 1 \mathrm{H}) ; 4.31(\mathrm{~d}, J=12.3 \mathrm{~Hz}, 1 \mathrm{H}) ; 4.56(\mathrm{~d}, J=$ $18.7 \mathrm{~Hz}, 1 \mathrm{H}) ; 4.71(\mathrm{~s}, 1 \mathrm{H}) ; 4.98\left(\mathrm{dd}, J_{1}=10.6 \mathrm{~Hz}, J_{2}=1.7\right.$ $\mathrm{Hz}, 1 \mathrm{H}) ; 5.16\left(\mathrm{dd}, J_{1}=17.3 \mathrm{~Hz}, J_{2}=1.7 \mathrm{~Hz}, 1 \mathrm{H}\right) ; 5.36(\mathrm{~m}$, $1 \mathrm{H}) ; 6.05\left(\mathrm{dd}, J_{1}=17.2 \mathrm{~Hz}, J_{2}=10.6 \mathrm{~Hz}, 1 \mathrm{H}\right) ; 7.08(\mathrm{~m}$, $1 \mathrm{H}) ; 7.14-7.24(\mathrm{~m}, 2 \mathrm{H}) ; 7.26-7.45(\mathrm{~m}, 2 \mathrm{H}) .{ }^{13} \mathrm{C}$ NMR $(75$ $\left.\mathrm{MHz}, \mathrm{CDCl}_{3}\right): \delta 12.0\left(\mathrm{CH}_{3}\right) ; 22.3\left(\mathrm{CH}_{3}\right) ; 22.5\left(\mathrm{CH}_{3}\right) ; 25.0$ $\left(\mathrm{CH}_{2}\right) ; 26.3\left(\mathrm{CH}_{3}\right) ; 31.5(\mathrm{CH}) ; 35.2\left(\mathrm{CH}_{2}\right) ; 41.4\left(\mathrm{CH}_{2}\right)$; $44.6(\mathrm{CH}) ; 46.6\left(\mathrm{CH}_{2}\right) ; 58.12(\mathrm{C}) ; 77.2\left(\mathrm{CH}_{2}\right) ; 77.9(\mathrm{CH})$; $89.9(\mathrm{CH}) ; 94.5(\mathrm{C}) ; 112.3\left(\mathrm{CH}_{2}\right) ; 123.6(\mathrm{CH}) ; 125.0(\mathrm{CH})$; $126.8(2 \mathrm{CH}) ; 127.3(2 \mathrm{CH}) ; 135.9(\mathrm{C}) ; 141.0(\mathrm{CH}) ; 144.6$ (C). IR (film): $3020,1600,720,695 \mathrm{~cm}^{-1}$. HRMS calcd for $\mathrm{C}_{25} \mathrm{H}_{36} \mathrm{NO}_{2}[\mathrm{M}+\mathrm{H}]^{+}$382.2741, found 382.2744

(2S,4aS,7R,8aR)-3-benzyl-4,4,7-trimethyl-2-( $(R)-3-$ methyl-2-(prop-1-en-2-yl)-2,5-dihydrofuran-2yl)octahydro- $2 H$-benzo $[e][1,3]$ oxazine $\quad(7 \mathrm{c})$. compound was obtained from $4 \mathrm{c}(1.2 \mathrm{~g})$ and catalyst 6 , and purified by flash chromatography (hexanes/ ethyl acetate 45:1). Yield: $1.03 \mathrm{~g}, 92 \%$. White solid. Mp 160-162 ${ }^{\circ} \mathrm{C}$ (from hexane). $[\alpha]_{\mathrm{D}}{ }^{25}=-64.2\left(\mathrm{c}=1, \mathrm{CHCl}_{3}\right) .{ }^{1} \mathrm{H}$ NMR $(300$ $\left.\mathrm{MHz}_{2} \mathrm{CDCl}_{3}\right): \delta 0.83-101(\mathrm{~m}, 2 \mathrm{H}) ; 0.84(\mathrm{~s}, 3 \mathrm{H}) ; 0.92(\mathrm{~d}, J$ $=6.5 \mathrm{~Hz}, 3 \mathrm{H}) ; 1.13(\mathrm{~m}, 1 \mathrm{H}) ; 1.29(\mathrm{~s}, 3 \mathrm{H}) ; 1.39-1.70(\mathrm{~m}$, $4 \mathrm{H}) ; 1.72(\mathrm{~s}, 3 \mathrm{H}) ; 1.79(\mathrm{~d}, J=1.7 \mathrm{~Hz}, 3 \mathrm{H}) ; 1.86(\mathrm{~m}, 1 \mathrm{H})$; $3.46\left(\mathrm{td}, J_{1}=10.5 \mathrm{~Hz}, J_{2}=4.0 \mathrm{~Hz}, 1 \mathrm{H}\right) ; 3.84(\mathrm{~d}, J=17.7$ $\mathrm{Hz}, 1 \mathrm{H}) ; 4.44(\mathrm{~m}, 1 \mathrm{H}) ; 4.57(\mathrm{~m}, 1 \mathrm{H}) ; 4.82(\mathrm{~d}, J=1.7 \mathrm{~Hz}$, $1 \mathrm{H}) ; 4.98(\mathrm{~d}, J=17.7 \mathrm{~Hz}, 1 \mathrm{H}) ; 4.99(\mathrm{~d}, J=1.7 \mathrm{~Hz}, 1 \mathrm{H})$; $5.06(\mathrm{~s}, 1 \mathrm{H}) ; 5.48(\mathrm{~m}, 1 \mathrm{H}) ; 7.08(\mathrm{~m}, 1 \mathrm{H}) ; 7.17-7.25(\mathrm{~m}$ $2 \mathrm{H}) ; 7.35-7.37(\mathrm{~m}, 2 \mathrm{H}) .{ }^{13} \mathrm{C}$ NMR $\left(75 \mathrm{MHz}, \mathrm{CDCl}_{3}\right): \delta$ $13.0\left(\mathrm{CH}_{3}\right) ; 20.0\left(\mathrm{CH}_{3}\right) ; 22.2\left(2 \mathrm{CH}_{3}\right) ; 24.9\left(\mathrm{CH}_{2}\right) ; 27.2$ $\left(\mathrm{CH}_{3}\right) ; 31.5(\mathrm{CH}) ; 35.1\left(\mathrm{CH}_{2}\right) ; 41.3\left(\mathrm{CH}_{2}\right) ; 43.9(\mathrm{CH}) ; 47.6$ $\left(\mathrm{CH}_{2}\right) ; 58.4(\mathrm{C}) ; 74.5\left(\mathrm{CH}_{2}\right) ; 77.9(\mathrm{CH}) ; 85.9(\mathrm{CH}) ; 96.0$ (C); $111.7\left(\mathrm{CH}_{2}\right) ; 121.3(\mathrm{CH}) ; 124.9(\mathrm{CH}) ; 127.1(2 \mathrm{CH})$; 127.3 (2 CH); 138.9 (C); 145.0 (C); 145.5 (C). IR (Nujol dispersion): $3060,1640,720,695 \mathrm{~cm}^{-1}$. HRMS calcd for $\mathrm{C}_{26} \mathrm{H}_{38} \mathrm{NO}_{2}[\mathrm{M}+\mathrm{H}]^{+}$396.2897, found 396.2906.

(2S,4aS,7R,8a $R)$-3-benzyl-2-((R)-3,4-dimethyl-2-(prop1-en-2-yl)-2,5-dihydrofuran-2-yl)-4,4,7-

trimethyloctahydro-2H-benzo $[e][1,3]$ oxazine $(7 d)$. This compound was obtained from $\mathbf{4 d}(1.2 \mathrm{~g})$ and catalyst $\mathbf{6}$, and purified by flash chromatography (hexanes/ ethyl acetate 60:1). Yield: $0.82 \mathrm{~g}, 73 \%$. Colorless oil. $[\alpha]_{\mathrm{D}}{ }^{25}=$ $74.7\left(\mathrm{c}=1, \mathrm{CH}_{2} \mathrm{Cl}_{2}\right) .{ }^{1} \mathrm{H} \mathrm{NMR}\left(300 \mathrm{MHz}, \mathrm{CDCl}_{3}\right): \delta 0.83$ $(\mathrm{s}, 3 \mathrm{H}) ; 0.84-1.06(\mathrm{~m}, 2 \mathrm{H}) ; 0.92(\mathrm{~d}, J=6.5 \mathrm{~Hz}, 3 \mathrm{H}) ; 1.14$ $(\mathrm{m}, 1 \mathrm{H}) ; 1.28(\mathrm{~s}, 3 \mathrm{H}) ; 1.39-1.72(\mathrm{~m}, 4 \mathrm{H}) ; 1.56(\mathrm{~s}, 3 \mathrm{H})$; $1.66(\mathrm{~d}, J=1.0 \mathrm{~Hz}, 3 \mathrm{H}) ; 1.71(\mathrm{~s}, 3 \mathrm{H}) ; 1.84(\mathrm{~m}, 1 \mathrm{H}) ; 3.43$ $\left(\mathrm{td}, J_{1}=10.5 \mathrm{~Hz}, J_{2}=4.0 \mathrm{~Hz}, 1 \mathrm{H}\right) ; 3.86(\mathrm{~d}, J=17.5 \mathrm{~Hz}$, $1 \mathrm{H}) ; 4.32-4.45(\mathrm{~m}, 2 \mathrm{H}) ; 4.78(\mathrm{~m}, 1 \mathrm{H}) ; 4.92(\mathrm{~d}, 1 \mathrm{H}, J=$ $17.5 \mathrm{~Hz}) ; 4.95(\mathrm{~s}, 1 \mathrm{H}) ; 5.03(\mathrm{~s}, 1 \mathrm{H}) ; 7.07(\mathrm{~m}, 1 \mathrm{H}) ; 7.16-$ $7.21(\mathrm{~m}, 2 \mathrm{H}) ; 7.35-7.37(\mathrm{~m}, 2 \mathrm{H}) .{ }^{13} \mathrm{C}$ NMR $(75 \mathrm{MHz}$ $\left.\mathrm{CDCl}_{3}\right): \delta$ 10.1 $\left(\mathrm{CH}_{3}\right) ; 10.5\left(\mathrm{CH}_{3}\right) ; 20.1\left(\mathrm{CH}_{3}\right) ; 22.1$ $\left(\mathrm{CH}_{3}\right) ; 22.3\left(\mathrm{CH}_{3}\right) ; 25.0\left(\mathrm{CH}_{2}\right) ; 27.2\left(\mathrm{CH}_{3}\right) ; 31.5(\mathrm{CH})$; $35.2\left(\mathrm{CH}_{2}\right) ; 41.4\left(\mathrm{CH}_{2}\right) ; 44.1(\mathrm{CH}) ; 47.8\left(\mathrm{CH}_{2}\right) ; 58.5(\mathrm{C})$; $77.8(\mathrm{CH}) ; 78.4\left(\mathrm{CH}_{2}\right) ; 86.4(\mathrm{CH}) ; 97.4(\mathrm{C}) ; 111.2\left(\mathrm{CH}_{2}\right)$; $124.9(\mathrm{CH}) ; 127.1(2 \mathrm{CH}) ; 127.2(2 \mathrm{CH}) ; 128.2(\mathrm{C}) ; 129.9$ (C); 145.5 (C); 145.6 (C). IR (film): 3020, 1600, 720, 695 $\mathrm{cm}^{-1}$. HRMS calcd for $\mathrm{C}_{27} \mathrm{H}_{40} \mathrm{NO}_{2}[\mathrm{M}+\mathrm{H}]^{+} 410.3054$, found 410.3058

$(2 S, 4 \mathrm{a} S, 7 R, 8 \mathrm{a} R)-3$-benzyl-2-( $(S)-3,4-d i m e t h y l-2-(p r o p-$ 1-en-2-yl)-2,5-dihydrofuran-2-yl)-4,4,7trimethyloctahydro- $2 H$-benzo $[e][1,3]$ oxazine $(8 d)$. This compound was obtained from $4 \mathbf{d}(1.2 \mathrm{~g})$ and catalyst $\mathbf{6}$, and purified by flash chromatography (hexanes/ ethyl acetate 60:1). Yield: $0.15 \mathrm{~g}, 13 \%$. White solid. Mp 128$130{ }^{\circ} \mathrm{C}$ (from hexane). $[\alpha]_{\mathrm{D}}{ }^{25}=+64.3\left(\mathrm{c}=1.5, \mathrm{CHCl}_{3}\right) .{ }^{1} \mathrm{H}$
NMR (300 MHz, $\left.\mathrm{CDCl}_{3}\right): \delta \quad 0.83-1.03(\mathrm{~m}, 2 \mathrm{H}) ; 0.89(\mathrm{~s}$, $3 \mathrm{H}) ; 0.90(\mathrm{~d}, J=6.6 \mathrm{~Hz}, 3 \mathrm{H}) ; 0.92(\mathrm{~s}, 3 \mathrm{H}) ; 1.21(\mathrm{~m}, 1 \mathrm{H})$ $1.34(\mathrm{~s}, 3 \mathrm{H}) ; 1.42-1.74(\mathrm{~m}, 4 \mathrm{H}) ; 1.65(\mathrm{~s}, 3 \mathrm{H}) ; 1.67(\mathrm{~s}, 3 \mathrm{H})$ $1.93(\mathrm{~m}, 1 \mathrm{H}) ; 3.50\left(\mathrm{td}, J_{1}=10.5 \mathrm{~Hz}, J_{2}=3.9 \mathrm{~Hz}, 1 \mathrm{H}\right) ; 3.86$ $(\mathrm{d}, J=19.0 \mathrm{~Hz}, 1 \mathrm{H}) ; 3.91(\mathrm{~m}, 1 \mathrm{H}) ; 4.26(\mathrm{~m}, 1 \mathrm{H}) ; 4.77(\mathrm{~d}, J$ $=19.0 \mathrm{~Hz}, 1 \mathrm{H}) ; 4.89-4.90(\mathrm{~m}, 2 \mathrm{H}) ; 5.14(\mathrm{~s}, 1 \mathrm{H}) ; 7.05(\mathrm{~m}$, $1 \mathrm{H})$; 7.15-7.21 (m, 2H); 7.25-7.27 (m, 2H). ${ }^{13} \mathrm{C}$ NMR $(75$ $\left.\mathrm{MHz}, \mathrm{CDCl}_{3}\right): \delta 9.3\left(\mathrm{CH}_{3}\right) ; 10.3\left(\mathrm{CH}_{3}\right) ; 19.7\left(\mathrm{CH}_{3}\right) ; 22.2$ $\left(\mathrm{CH}_{3}\right) ; 22.7\left(\mathrm{CH}_{3}\right) ; 25.0\left(\mathrm{CH}_{2}\right) ; 26.2\left(\mathrm{CH}_{3}\right) ; 31.5(\mathrm{CH})$ $35.2\left(\mathrm{CH}_{2}\right) ; 41.4\left(\mathrm{CH}_{2}\right) ; 43.8(\mathrm{CH}) ; 46.2\left(\mathrm{CH}_{2}\right) ; 58.5(\mathrm{C})$; $78.1(\mathrm{CH}) ; 78.3\left(\mathrm{CH}_{2}\right) ; 86.2(\mathrm{CH}) ; 97.4(\mathrm{C}) ; 109.6\left(\mathrm{CH}_{2}\right)$ $124.8(\mathrm{CH}) ; 126.1(2 \mathrm{CH}) ; 127.2(2 \mathrm{CH}) ; 128.6(\mathrm{C}) ; 128.8$ (C); 145.2 (C); 148.5 (C). IR (Nujol dispersion): 3085 , $1600,720,695 \mathrm{~cm}^{-1}$. HRMS calcd for $\mathrm{C}_{27} \mathrm{H}_{40} \mathrm{NO}_{2}[\mathrm{M}+\mathrm{H}]$ 410.3054 , found 410.3062

$(2 S, 4 a S, 7 R, 8 \mathrm{a} R)-2-((R)-2-a l l y l-3,6-d i h y d r o-2 H$-pyran-2yl)-3-benzyl-4,4,7-trimethyloctahydro- $2 H$ -

benzo $[e][1,3]$ oxazine $(7 \mathrm{e})$. This compound was obtained from $4 \mathrm{e}(1.2 \mathrm{~g})$ and catalyst $\mathbf{5}$, and purified by flash chromatography (hexanes/ ethyl acetate 60:1). Yield: 0.67 g, $60 \%$. Colorless oil. $[\alpha]_{\mathrm{D}}{ }^{25}=+27.1\left(\mathrm{c}=2.0, \mathrm{CH}_{2} \mathrm{Cl}_{2}\right) .{ }^{\mathrm{T}} \mathrm{H}$ NMR (300 MHz, $\left.\mathrm{CDCl}_{3}\right): \delta 0.87-1.04(\mathrm{~m}, 2 \mathrm{H}) ; 0.92(\mathrm{~d}, J=$ $6.4 \mathrm{~Hz}, 3 \mathrm{H}) ; 0.93(\mathrm{~s}, 3 \mathrm{H}) ; 1.24(\mathrm{~m}, 1 \mathrm{H}) ; 1.29(\mathrm{~s}, 3 \mathrm{H}) ; 1.47$ $(\mathrm{m}, 1 \mathrm{H}) ; 1.55-1.78(\mathrm{~m}, 3 \mathrm{H}) ; 1.81-2.01(\mathrm{~m}, 2 \mathrm{H}) ; 2.25(\mathrm{~m}$, $1 \mathrm{H}) ; 2.40(\mathrm{~m}, 1 \mathrm{H}) ; 2.55(\mathrm{~m}, 1 \mathrm{H}) ; 3.46\left(\mathrm{td}, J_{1}=10.6 \mathrm{~Hz}, J_{2}\right.$ $=3.8 \mathrm{~Hz}, 1 \mathrm{H}) ; 3.86-3.99(\mathrm{~m}, 2 \mathrm{H}) ; 4.12(\mathrm{~m}, 1 \mathrm{H}) ; 4.38(\mathrm{~s}$ $1 \mathrm{H}) ; 5.02-5.08(\mathrm{~m}, 3 \mathrm{H}) ; 5.62(\mathrm{~m}, 1 \mathrm{H}) ; 5.70(\mathrm{~m}, 1 \mathrm{H}) ; 6.12$ $(\mathrm{m}, 1 \mathrm{H}) ; 7.13(\mathrm{~m}, 1 \mathrm{H}) ; 7.24-7.29(\mathrm{~m}, 2 \mathrm{H}) ; 7.40-7.43(\mathrm{~m}$ $2 \mathrm{H}) .{ }^{13} \mathrm{C}$ NMR $\left(75 \mathrm{MHz}, \mathrm{CDCl}_{3}\right): \delta 22.3\left(2 \mathrm{CH}_{3}\right) ; 25.0$ $\left(\mathrm{CH}_{2}\right) ; 27.0\left(\mathrm{CH}_{3}\right) ; 28.5\left(\mathrm{CH}_{2}\right) ; 31.6(\mathrm{CH}) ; 35.2\left(\mathrm{CH}_{2}\right)$ $35.4\left(\mathrm{CH}_{2}\right) ; 41.5\left(\mathrm{CH}_{2}\right) ; 44.1(\mathrm{CH}) ; 47.9\left(\mathrm{CH}_{2}\right) ; 58.6(\mathrm{C})$ $60.7\left(\mathrm{CH}_{2}\right) ; 76.4(\mathrm{C}) ; 78.3(\mathrm{CH}) ; 89.4(\mathrm{CH}) ; 118.0\left(\mathrm{CH}_{2}\right)$ $123.1(\mathrm{CH}) ; 124.9(\mathrm{CH}) ; 125.2(\mathrm{CH}) ; 126.8(2 \mathrm{CH}) ; 127.8$ $(2 \mathrm{CH}) ; 134.9(\mathrm{CH}) ; 145.2$ (C). IR (film): 3060, 1740 $1640,730,670 \mathrm{~cm}^{-1}$. HRMS calcd for $\mathrm{C}_{26} \mathrm{H}_{38} \mathrm{NO}_{2}[\mathrm{M}+\mathrm{H}]^{+}$ 396.2897, found 396.2893

(2S,4aS,7R,8a $R)-2-((S)-2$-allyl-3,6-dihydro-2H-pyran-2yl)-3-benzyl-4,4,7-trimethyloctahydro- $2 \mathrm{H}$ -

benzo $[e][1,3]$ oxazine $(\mathbf{8 e})$. This compound was obtained from $\left.4 e^{(1.2} \mathrm{g}\right)$ and catalyst $\mathbf{6}$, and purified by flash chromatography (hexanes/ ethyl acetate 60:1). Yield: 0.71 g, $63 \%$. White solid. Mp $76-78^{\circ} \mathrm{C}$ (from pentane). $[\alpha]_{\mathrm{D}}{ }^{25}=$ $-18.7\left(\mathrm{c}=1.5, \mathrm{CH}_{2} \mathrm{Cl}_{2}\right) \cdot{ }^{1} \mathrm{H}$ NMR $\left(300 \mathrm{MHz}, \mathrm{CDCl}_{3}\right): \delta$ $0.89-1.06(\mathrm{~m}, 2 \mathrm{H}) ; 94(\mathrm{~d}, J=6.5 \mathrm{~Hz}, 3 \mathrm{H}) ; 0.99(\mathrm{~s}, 3 \mathrm{H})$; $1.23(\mathrm{~m}, 1 \mathrm{H}) ; 1.27(\mathrm{~s}, 3 \mathrm{H}) ; 1.40-1.75(\mathrm{~m}, 5 \mathrm{H}) ; 1.95(\mathrm{~m}$ $1 \mathrm{H}) ; 2.39\left(\mathrm{dd}, J_{1}=13.4 \mathrm{~Hz}, J_{2}=6.9 \mathrm{~Hz}, 1 \mathrm{H}\right) ; 2.51\left(\mathrm{dd}, J_{1}=\right.$ $\left.17.4 \mathrm{~Hz}, J_{2}=7.6 \mathrm{~Hz}, 1 \mathrm{H}\right) ; 2.70(\mathrm{~m}, 1 \mathrm{H}) ; 3.49\left(\mathrm{td}, J_{1}=10.8\right.$ $\left.\mathrm{Hz}, J_{2}=3.8 \mathrm{~Hz}, 1 \mathrm{H}\right) ; 3.89(\mathrm{~d}, J=18.1 \mathrm{~Hz}, 1 \mathrm{H}) ; 3.95(\mathrm{~m}$ $1 \mathrm{H}) ; 4.09(\mathrm{~m}, 1 \mathrm{H}) ; 4.55(\mathrm{~s}, 1 \mathrm{H}) ; 4.68(\mathrm{~d}, J=18.1 \mathrm{~Hz}, 1 \mathrm{H})$ $5.12(\mathrm{~m}, 2 \mathrm{H}) ; 5.48(\mathrm{~m}, 1 \mathrm{H}) ; 5.55(\mathrm{~m}, 1 \mathrm{H}) ; 5.89(\mathrm{~m}, 1 \mathrm{H})$ $7.14(\mathrm{~m}, 1 \mathrm{H}) ; 7.20-7.29(\mathrm{~m}, 2 \mathrm{H}) ; 7.31-7.42(\mathrm{~m}, 2 \mathrm{H}) .{ }^{13} \mathrm{C}$ NMR $\left(75 \mathrm{MHz}, \mathrm{CDCl}_{3}\right): \delta 22.3\left(\mathrm{CH}_{3}\right) ; 23.0\left(\mathrm{CH}_{3}\right) ; 25.0$ $\left(\mathrm{CH}_{2}\right) ; 27.0\left(\mathrm{CH}_{3}\right) ; 27.4\left(\mathrm{CH}_{2}\right) ; 31.5(\mathrm{CH}) ; 35.2\left(\mathrm{CH}_{2}\right)$; $37.0\left(\mathrm{CH}_{2}\right) ; 41.4\left(\mathrm{CH}_{2}\right) ; 44.5(\mathrm{CH}) ; 47.5\left(\mathrm{CH}_{2}\right) ; 58.8(\mathrm{C})$ $60.5\left(\mathrm{CH}_{2}\right) ; 76.6(\mathrm{C}) ; 78.0(\mathrm{CH}) ; 88.9(\mathrm{CH}) ; 117.8\left(\mathrm{CH}_{2}\right)$ $122.2(\mathrm{CH}) ; 124.4(\mathrm{CH}) ; 125.1(\mathrm{CH}) ; 127.0(2 \mathrm{CH}) ; 127.6$ $(2 \mathrm{CH}) ; 134.6(\mathrm{CH}) ; 144.6(\mathrm{C})$. IR (film): 3060, 1640, 720, $670 \mathrm{~cm}^{-1}$. HRMS calcd for $\mathrm{C}_{26} \mathrm{H}_{38} \mathrm{NO}_{2}[\mathrm{M}+\mathrm{H}]^{+} 396.2897$, found 396.2895

\section{$(2 S, 4 a S, 7 R, 8 a R)-3-b e n z y l-4,4,7-t r i m e t h y l-2-((R)-4-$ methyl-2-(2-methylallyl)-3,6-dihydro-2H-pyran-2-} yl)octahydro-2H-benzo[ $e][1,3]$ oxazine $(7 f)$.

compound was obtained from $\mathbf{4 f}(1.2 \mathrm{~g})$ and catalyst $\mathbf{5}$, and purified by flash chromatography (hexanes/ ethyl acetate 50:1). Yield: $0.82 \mathrm{~g}, 73 \%$. Colorless oil. $[\alpha]_{\mathrm{D}}{ }^{25}=+21.6(\mathrm{c}=$ $\left.1.8, \mathrm{CH}_{2} \mathrm{Cl}_{2}\right)$. $\mathrm{H}$ NMR $\left(300 \mathrm{MHz}, \mathrm{CDCl}_{3}\right): \delta 0.78-0.96(\mathrm{~m}$, $2 \mathrm{H}) ; 0.85(\mathrm{~d}, J=6.5 \mathrm{~Hz}, 3 \mathrm{H}) ; 0.91(\mathrm{~s}, 3 \mathrm{H}) ; 1.08(\mathrm{~m}, 1 \mathrm{H})$ $1.16(\mathrm{~s}, 3 \mathrm{H}) ; 1.28-1.65(\mathrm{~m}, 5 \mathrm{H}) ; 1.52(\mathrm{~s}, 3 \mathrm{H}) ; 1.70(\mathrm{~s}, 3 \mathrm{H})$ $1.82(\mathrm{~m}, 1 \mathrm{H}) ; 2.16(\mathrm{~d}, J=13.7 \mathrm{~Hz}, 1 \mathrm{H}) ; 2.29(\mathrm{~d}, J=13.7$ $\mathrm{Hz}, 1 \mathrm{H}) ; 2.45(\mathrm{~m}, 1 \mathrm{H}) ; 3.38\left(\mathrm{td}, J_{1}=10.4 \mathrm{~Hz}, J_{2}=3.9 \mathrm{~Hz}\right.$ $1 \mathrm{H}) ; 3.81-3.91(\mathrm{~m}, 2 \mathrm{H}) ; 4.00(\mathrm{~m}, 1 \mathrm{H}) ; 4.50(\mathrm{~s}, 1 \mathrm{H}) ; 4.64$ $(\mathrm{m}, 1 \mathrm{H}) ; 4.78-4.80(\mathrm{~m}, 2 \mathrm{H}) ; 5.14(\mathrm{~m}, 1 \mathrm{H}) ; 7.05(\mathrm{~m}, 1 \mathrm{H})$ 7.14-7.23 (m, 2H); 7.27-7.43 (m, 2H). ${ }^{13} \mathrm{C}$ NMR $(75 \mathrm{MHz}$ $\left.\mathrm{CDCl}_{3}\right): \delta 20.7\left(\mathrm{CH}_{3}\right) ; 22.2\left(\mathrm{CH}_{3}\right) ; 23.0\left(\mathrm{CH}_{3}\right) ; 24.7\left(\mathrm{CH}_{3}\right)$ $25.2\left(\mathrm{CH}_{2}\right) ; 27.2\left(\mathrm{CH}_{3}\right) ; 31.5(\mathrm{CH}) ; 34.0\left(\mathrm{CH}_{2}\right) ; 35.2$ $\left(\mathrm{CH}_{2}\right) ; 39.4\left(\mathrm{CH}_{2}\right) ; 41.6\left(\mathrm{CH}_{2}\right) ; 46.0(\mathrm{CH}) ; 48.0\left(\mathrm{CH}_{2}\right)$ $58.9(\mathrm{C}) ; 60.8\left(\mathrm{CH}_{2}\right) ; 77.2(\mathrm{C}) ; 77.3(\mathrm{CH}) ; 89.7(\mathrm{CH})$; 
$114.4\left(\mathrm{CH}_{2}\right) ; 117.8(\mathrm{CH}) ; 125.1(\mathrm{CH}) ; 127.2(2 \mathrm{CH}) ; 127.5$ (2 CH); 130.7 (C); 142.9 (C); 145.0 (C). IR (film): 3060, $1640,730,700 \mathrm{~cm}^{-1}$. HRMS calcd for $\mathrm{C}_{28} \mathrm{H}_{42} \mathrm{NO}_{2}[\mathrm{M}+\mathrm{H}]^{+}$ 424.3210, found 424.3207 .

\section{$(2 S, 4 \mathrm{a} S, 7 R, 8 \mathrm{a} R)-3-$ benzyl-4,4,7-trimethyl-2-( $(S)-4-$} methyl-2-(2-methylallyl)-3,6-dihydro-2H-pyran-2yl)octahydro- $2 H$-benzo $[e][1,3]$ oxazine $(8 f)$.

This compound was obtained from $\mathbf{4 f}(1.2 \mathrm{~g})$ and catalyst $\mathbf{6}$, and purified by flash chromatography (hexanes/ ethyl acetate 50:1). Yield: 0,66 g, 59\%. White solid. Mp 93-95 ${ }^{\circ} \mathrm{C}$ (from hexane $) .[\alpha]_{\mathrm{D}}{ }^{25}=-52.0\left(\mathrm{c}=2.0, \mathrm{CH}_{2} \mathrm{Cl}_{2}\right) .{ }^{1} \mathrm{H}$ NMR $(300$ $\left.\mathrm{MHz}, \mathrm{CDCl}_{3}\right): \delta 0.83-1.02(\mathrm{~m}, 2 \mathrm{H}) ; 0.93(\mathrm{~d}, J=6.4 \mathrm{~Hz}$, $3 \mathrm{H}) ; 0.98(\mathrm{~s}, 3 \mathrm{H}) ; 1.23(\mathrm{~m}, 1 \mathrm{H}) ; 1.25(\mathrm{~s}, 3 \mathrm{H}) ; 1.41-1.74(\mathrm{~m}$, $5 \mathrm{H}) ; 1.52(\mathrm{~s}, 3 \mathrm{H}) ; 1.78(\mathrm{~s}, 3 \mathrm{H}) ; 1.90(\mathrm{~m}, 1 \mathrm{H}) ; 2.31(\mathrm{~d}, 1 \mathrm{H}$, $J=13.2 \mathrm{~Hz}) ; 2.55(\mathrm{~d}, 1 \mathrm{H}, J=13.2 \mathrm{~Hz}) ; 2.62(\mathrm{~m}, 1 \mathrm{H}) ; 3.48$ $\left(\mathrm{td}, J_{1}=10.6 \mathrm{~Hz}, J_{2}=3.9 \mathrm{~Hz}, 1 \mathrm{H}\right) ; 3.89(\mathrm{~d}, J=17.9 \mathrm{~Hz}$, $1 \mathrm{H}) ; 3.94(\mathrm{~m}, 1 \mathrm{H}) ; 4.06(\mathrm{~m}, 1 \mathrm{H}) ; 4.62(\mathrm{~s}, 1 \mathrm{H}) ; 4.78(\mathrm{~d}, J=$ $0.9 \mathrm{~Hz}, 1 \mathrm{H}) ; 4.84(\mathrm{~d}, J=17.9 \mathrm{~Hz}, 1 \mathrm{H}) ; 4.87(\mathrm{~d}, J=0.9 \mathrm{~Hz}$, $1 \mathrm{H}) ; 5.23(\mathrm{~m}, 1 \mathrm{H}) ; 7.11(\mathrm{~m}, 1 \mathrm{H}) ; 7.19-7.28(\mathrm{~m}, 2 \mathrm{H}) ; 7.31-$ $7.42(\mathrm{~m}, 2 \mathrm{H}) .{ }^{13} \mathrm{C}$ NMR $\left(75 \mathrm{MHz}, \mathrm{CDCl}_{3}\right): 22.2\left(\mathrm{CH}_{3}\right)$; $22.9\left(\mathrm{CH}_{3}\right) ; 23.0\left(\mathrm{CH}_{3}\right) ; 24.5\left(\mathrm{CH}_{3}\right) ; 25.2\left(\mathrm{CH}_{2}\right) ; 27.2$ $\left(\mathrm{CH}_{3}\right) ; 31.7(\mathrm{CH}) ; 32.8\left(\mathrm{CH}_{2}\right) ; 35.4\left(\mathrm{CH}_{2}\right) ; 40.1\left(\mathrm{CH}_{2}\right)$; $41.6\left(\mathrm{CH}_{2}\right) ; 44.9(\mathrm{CH}) ; 47.7\left(\mathrm{CH}_{2}\right) ; 58.9(\mathrm{C}) ; 60.9\left(\mathrm{CH}_{2}\right)$; $77.6(\mathrm{C}) ; 77.7(\mathrm{CH}) ; 88.2(\mathrm{CH}) ; 114.6\left(\mathrm{CH}_{2}\right) ; 117.9(\mathrm{CH})$; $125.1(\mathrm{CH}) ; 127.2(2 \mathrm{CH}) ; 127.5(2 \mathrm{CH}) ; 129.7(\mathrm{C}) ; 143.0$ (C); 144.9 (C).IR (nujol dispersion): 3060, 1640, 730, 700 $\mathrm{cm}^{-1}$. HRMS calcd for $\mathrm{C}_{28} \mathrm{H}_{42} \mathrm{NO}_{2}[\mathrm{M}+\mathrm{H}]^{+}$424.3210, found 424.3210

\section{$(2 S, 4 a S, 7 R, 8 a R)-3-b e n z y l-2-((R)-4,5-d i m e t h y l-2-(2-$} methylallyl)-3,6-dihydro-2H-pyran-2-yl)-4,4,7-

trimethyloctahydro-2H-benzo[e][1,3]oxazine $(7 \mathrm{~g})$. This compound was obtained from $\mathbf{4 g}(1.2 \mathrm{~g})$ and catalyst $\mathbf{6}$, and purified by flash chromatography (hexanes/ ethyl acetate 60:1). Yield: $0.68 \mathrm{~g}, 60 \%$. Colorless oil. $[\alpha]_{\mathrm{D}}{ }^{25}=+17.0(\mathrm{c}=$ $\left.1.0, \mathrm{CH}_{2} \mathrm{Cl}_{2}\right)$. $\mathrm{H}$ NMR $\left(300 \mathrm{MHz}, \mathrm{CDCl}_{3}\right): \delta 0.84-1.05(\mathrm{~m}$, $2 \mathrm{H}) ; 0,93(\mathrm{~d}, J=6.5 \mathrm{~Hz}, 3 \mathrm{H}) ; 0.99(\mathrm{~s}, 3 \mathrm{H}) ; 1.20(\mathrm{~m}, 1 \mathrm{H})$; $1.23(\mathrm{~s}, 3 \mathrm{H}) ; 1.35-1.73(\mathrm{~m}, 5 \mathrm{H}) ; 1.40(\mathrm{~s}, 3 \mathrm{H}) ; 1.54(\mathrm{~s}, 3 \mathrm{H})$; $1.76(\mathrm{~s}, 3 \mathrm{H}) ; 1.94(\mathrm{~m}, 1 \mathrm{H}) ; 2.22(\mathrm{~d}, J=13.8 \mathrm{~Hz}, 1 \mathrm{H}) ; 2.35$ $(\mathrm{d}, J=13.8 \mathrm{~Hz}, 1 \mathrm{H}) ; 2.52(\mathrm{~m}, 1 \mathrm{H}) ; 3.45\left(\mathrm{td}, J_{l}=10.4 \mathrm{~Hz}\right.$, $\left.J_{2}=4.1 \mathrm{~Hz}, 1 \mathrm{H}\right) ; 3.76(\mathrm{~m}, 1 \mathrm{H}) ; 3.86(\mathrm{~m}, 1) ; 3.94(\mathrm{~d}, J=$ $17.8 \mathrm{~Hz}, 1 \mathrm{H}) ; 4.55(\mathrm{~s}, 1 \mathrm{H}) ; 4.74(\mathrm{~d}, J=17.8 \mathrm{~Hz}, 1 \mathrm{H}) ; 4.84$ $(\mathrm{s}, 2 \mathrm{H}) ; 7.12(\mathrm{~m}, 1 \mathrm{H}) ; 7.17-7.28(\mathrm{~m}, 2 \mathrm{H}) ; 7.38-7.44(\mathrm{~m}$, $2 \mathrm{H}){ }^{13} \mathrm{C}$ NMR $\left(75 \mathrm{MHz}, \mathrm{CDCl}_{3}\right): \delta 13.5\left(\mathrm{CH}_{3}\right) ; 18.4$ $\left(\mathrm{CH}_{3}\right) ; 20.8\left(\mathrm{CH}_{3}\right) ; 22.2\left(\mathrm{CH}_{3}\right) ; 24.7\left(\mathrm{CH}_{3}\right) ; 25.2\left(\mathrm{CH}_{2}\right) ;$ $27.3\left(\mathrm{CH}_{3}\right) ; 31.6(\mathrm{CH}) ; 34.9\left(\mathrm{CH}_{2}\right) ; 35.3\left(\mathrm{CH}_{2}\right) ; 39.5$ $\left(\mathrm{CH}_{2}\right) ; 41.7\left(\mathrm{CH}_{2}\right) ; 46.0(\mathrm{CH}) ; 48.1\left(\mathrm{CH}_{2}\right) ; 58.9(\mathrm{C}) ; 64.7$ $\left.\left(\mathrm{CH}_{2}\right) ; 77.3(\mathrm{CH}) ; 77.8(\mathrm{C}) ; 89.6(\mathrm{CH}) ; 114.3 \mathrm{CH}_{2}\right) ; 122.2$ (C); 122.7 (C); $125.1(\mathrm{CH}) ; 127.3(2 \mathrm{CH}) ; 127.5(2 \mathrm{CH})$; 143.1 (C); 145.1 (C). IR (film): 3065, 1635, 735, $700 \mathrm{~cm}^{-1}$ HRMS calcd for $\mathrm{C}_{29} \mathrm{H}_{44} \mathrm{NO}_{2}[\mathrm{M}+\mathrm{H}]^{+} 438.3367$, found 438.3369

\section{$(2 S, 4 \mathrm{a} S, 7 R, 8 \mathrm{a} R)-3$-benzyl-2-( $(S)-4,5$-dimethyl-2-(2- methylallyl)-3,6-dihydro-2H-pyran-2-yl)-4,4,7-}

trimethyloctahydro-2H-benzo $[e][1,3]$ oxazine $(8 \mathrm{~g})$. This compound was obtained from $\mathbf{4 g}(1.2 \mathrm{~g})$ and catalyst $\mathbf{6}$, and purified by flash chromatography (hexanes/ ethyl acetate 60:1). Yield: $0.25 \mathrm{~g}, 22 \%$. White solid. Mp 87-89 ${ }^{\circ} \mathrm{C}$ (from pentane). $[\alpha]_{\mathrm{D}}{ }^{25}=-61.5\left(\mathrm{c}=2.0, \mathrm{CH}_{2} \mathrm{Cl}_{2}\right) \cdot{ }^{1} \mathrm{H}$ NMR $(300$ $\left.\mathrm{MHz}, \mathrm{CDCl}_{3}\right): \delta 0.88-1.03(\mathrm{~m}, 2 \mathrm{H}) ; 0.93(\mathrm{~d}, J=6.5 \mathrm{~Hz}$, $3 \mathrm{H}) ; 0.95(\mathrm{~s}, 3 \mathrm{H}) ; 1.25(\mathrm{~s}, 3 \mathrm{H}) ; 1.35(\mathrm{~s}, 3 \mathrm{H}) ; 1.43-1.72(\mathrm{~m}$, $5 \mathrm{H}) ; 1.47(\mathrm{~s}, 3 \mathrm{H}) ; 1.77(\mathrm{~s}, 3 \mathrm{H}) ; 1.90(\mathrm{~m}, 1 \mathrm{H}) ; 2.28(\mathrm{~d}, J=$ $13.2 \mathrm{~Hz}, 1 \mathrm{H}) ; 2.53(\mathrm{~d}, J=13.2 \mathrm{~Hz}, 1 \mathrm{H}) ; 2.59(\mathrm{~m}, 1 \mathrm{H}) ; 3.47$ $\left(\mathrm{td}, J_{l}=10.5 \mathrm{~Hz}, J_{2}=3.9 \mathrm{~Hz}, 1 \mathrm{H}\right) ; 3.73(\mathrm{~m}, 1 \mathrm{H}) ; 3.89(\mathrm{~d}, J$ $=17.9 \mathrm{~Hz}, 1 \mathrm{H}) ; 3.90(\mathrm{~m}, 1 \mathrm{H}) ; 4.63(\mathrm{~s}, 1 \mathrm{H}) ; 4.77(\mathrm{~m}, 1 \mathrm{H})$; $4.82(\mathrm{~d}, J=17.9 \mathrm{~Hz}, 1 \mathrm{H}) ; 4.88(\mathrm{~m}, 1 \mathrm{H}) ; 7.09(\mathrm{~m}, 1 \mathrm{H})$; 7.20-7.25 (m, 2H); 7.26-7.39 (m, 2H). ${ }^{13} \mathrm{C}$ NMR (75 MHz, $\left.\mathrm{CDCl}_{3}\right): \delta 13.5\left(\mathrm{CH}_{3}\right) ; 18.4\left(\mathrm{CH}_{3}\right) ; 20.8\left(\mathrm{CH}_{3}\right) ; 22.2\left(\mathrm{CH}_{3}\right)$; $24.7\left(\mathrm{CH}_{3}\right) ; 25.2\left(\mathrm{CH}_{2}\right) ; 27.3\left(\mathrm{CH}_{3}\right) ; 31.6(\mathrm{CH}) ; 34.9$ $\left(\mathrm{CH}_{2}\right) ; 35.3\left(\mathrm{CH}_{2}\right) ; 39.5\left(\mathrm{CH}_{2}\right) ; 41.7\left(\mathrm{CH}_{2}\right) ; 46.0(\mathrm{CH})$; $48.1\left(\mathrm{CH}_{2}\right) ; 58.9(\mathrm{C}) ; 64.7\left(\mathrm{CH}_{2}\right) ; 77.3(\mathrm{CH}) ; 77.8(\mathrm{C})$; $89.6(\mathrm{CH}) ; 114.3\left(\mathrm{CH}_{2}\right) ; 122.2(\mathrm{C}) ; 122.7(\mathrm{C}) ; 125.1(\mathrm{CH})$; $127.3(2 \mathrm{CH}) ; 127.5$ (2 CH); 143.1 (C); 145.1 (C). IR (film): $3060,1635,725,695 \mathrm{~cm}^{-1}$. HRMS calcd for $\mathrm{C}_{29} \mathrm{H}_{44} \mathrm{NO}_{2}[\mathrm{M}+\mathrm{H}]^{+}$438.3367, found 438.3368.
$(2 S, 4 a S, 7 R, 8 a R)-3-b e n z y l-2-((R)-2-e t h y l-2,5-$ dihydrofuran-2-yl)-4,4,7-trimethyloctahydro-2H-

benzo $[e][1,3]$ oxazine (14). This compound was obtained from $13(2.0 \mathrm{~g})$ and catalyst 5 , and purified by flash chromatography (hexanes/ ethyl acetate 45:1). Yield: 1.63 g, $88 \%$. Colorless oil. $[\alpha]_{\mathrm{D}}{ }^{25}=+6.2\left(\mathrm{c}=0.8, \mathrm{CH}_{2} \mathrm{Cl}_{2}\right) .{ }^{1} \mathrm{H}$ NMR $\left(300 \mathrm{MHz}, \mathrm{CDCl}_{3}\right): \delta 0.82(\mathrm{t}, J=7.4 \mathrm{~Hz}, 3 \mathrm{H}) ; 0.84$ $1.00(\mathrm{~m}, 2 \mathrm{H}) ; 0.87(\mathrm{~s}, 3 \mathrm{H}) ; 0.92(\mathrm{~d}, J=6.5 \mathrm{~Hz}, 3 \mathrm{H}) ; 1.13$ $(\mathrm{m}, 1 \mathrm{H}) ; 1.28(\mathrm{~s}, 3 \mathrm{H}) ; 1.35-1.58(\mathrm{~m}, 2 \mathrm{H}) ; 1.60-1.73(\mathrm{~m}$ $4 \mathrm{H}) ; 1.86(\mathrm{~m}, 1 \mathrm{H}) ; 3.44\left(\mathrm{td}, J_{1}=10.6 \mathrm{~Hz}, J_{2}=3.9 \mathrm{~Hz}, 1 \mathrm{H}\right)$ $3.93(\mathrm{~d}, \mathrm{~J}=18.4 \mathrm{~Hz}, 1 \mathrm{H}) ; 4.48-4.51(\mathrm{~m}, 2 \mathrm{H}) ; 4.66(\mathrm{~s}, 1 \mathrm{H})$; $4.78(\mathrm{~d}, J=18.4 \mathrm{~Hz}, 1 \mathrm{H}) ; 5.65(\mathrm{~m}, 1 \mathrm{H}) ; 5.84(\mathrm{~m}, 1 \mathrm{H})$ $7.11(\mathrm{~m}, 1 \mathrm{H}) ; 7.22-7.27(\mathrm{~m}, 2 \mathrm{H}) ; 7.41-7.43(\mathrm{~m}, 2 \mathrm{H}) .{ }^{3} \mathrm{C}$ NMR (75 MHz, CDCl 3$): \delta 8.27\left(\mathrm{CH}_{3}\right) ; 22.3\left(\mathrm{CH}_{3}\right) ; 22.7$ $\left(\mathrm{CH}_{3}\right) ; 24.9\left(\mathrm{CH}_{2}\right) ; 26.8\left(\mathrm{CH}_{3}\right) ; 29.7\left(\mathrm{CH}_{2}\right) ; 31.5(\mathrm{CH})$ $35.2\left(\mathrm{CH}_{2}\right) ; 41.3\left(\mathrm{CH}_{2}\right) ; 43.8(\mathrm{CH}) ; 47.3\left(\mathrm{CH}_{2}\right) ; 57.9(\mathrm{C})$ $76.6\left(\mathrm{CH}_{2}\right) ; 77.6(\mathrm{CH}) ; 89.9(\mathrm{CH}) ; 94.5(\mathrm{C}) ; 125.2(\mathrm{CH})$ $126.7(2 \mathrm{CH}) ; 127.1(\mathrm{CH}) ; 127.6(2 \mathrm{CH}) ; 130.7(\mathrm{CH})$; 144.9 (C). IR (film): 3058, 1605, 722, $695 \mathrm{~cm}^{-1}$. HRMS calcd for $\mathrm{C}_{24} \mathrm{H}_{36} \mathrm{NO}_{2}[\mathrm{M}+\mathrm{H}]^{+} 370.2741$, found 370.2750

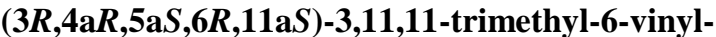

\section{$2,3,4,4 a, 5 a, 6,11,11 a-o c t a h y d r o-1 H, 9 H-$}

\section{benzo $[e]$ pyrido $[2,1-b][1,3]$ oxazin-6-ol $\quad(22 a)$.}

This

compound was obtained from $\mathbf{1 9}$ a $(1.0 \mathrm{~g})$ and catalyst $\mathbf{5}$ and purified by flash chromatography (hexanes/ ethyl acetate 20:1). Yield: $0.17 \mathrm{~g}, 19 \%$. White solid. Mp 136 $138^{\circ} \mathrm{C}$ (from hexane). $[\alpha]_{\mathrm{D}}{ }^{25}=-112.2\left(\mathrm{c}=1.2, \mathrm{CH}_{2} \mathrm{Cl}_{2}\right) .{ }^{1} \mathrm{H}$ NMR (300 MHz, CDCl $\left.{ }_{3}\right): \delta 0.90(\mathrm{~d}, J=6.4 \mathrm{~Hz}, 3 \mathrm{H}) ; 0.92-$ $1.25(\mathrm{~m}, 3 \mathrm{H}) ; 1.18(\mathrm{~s}, 3 \mathrm{H}) ; 1.22(\mathrm{~s}, 3 \mathrm{H}) ; 1.46(\mathrm{~m}, 1 \mathrm{H})$; $1.56-1.63(\mathrm{~m}, 2 \mathrm{H}) ; 1.71(\mathrm{~m}, 1 \mathrm{H}) ; 1.89(\mathrm{~m}, 1 \mathrm{H}) ; 2.70$ (broad, $1 \mathrm{H}) ; 3.26(\mathrm{~m}, 1 \mathrm{H}) ; 3.40\left(\mathrm{td}, J_{l}=10.7 \mathrm{~Hz}, J_{2}=3.9 \mathrm{~Hz}, 1 \mathrm{H}\right)$; $3.48(\mathrm{~m}, 1 \mathrm{H}) ; 4.52(\mathrm{~s}, 1 \mathrm{H}) ; 5.20\left(\mathrm{dd}, J_{l}=10.7 \mathrm{~Hz}, J_{2}=1.7\right.$ $\mathrm{Hz}, 1 \mathrm{H}) ; 5.46\left(\mathrm{dd}, J_{l}=17.3 \mathrm{~Hz}, J_{2}=1.7 \mathrm{~Hz}, 1 \mathrm{H}\right) ; 5.69(\mathrm{~d}$, $J=9.9 \mathrm{~Hz}, 1 \mathrm{H}) ; 5.89\left(\mathrm{ddd}, J_{l}=9.9 \mathrm{~Hz}, J_{2}=4.2 \mathrm{~Hz}, J_{\beta_{3}}=\right.$ $2.5 \mathrm{~Hz}, 1 \mathrm{H}) ; 6.06\left(\mathrm{dd}, J_{l}=17.3 \mathrm{~Hz}, J_{2}=10.7 \mathrm{~Hz}, 1 \mathrm{H}\right) \cdot{ }^{13} \mathrm{C}$ NMR $\left(75 \mathrm{MHz}, \mathrm{CDCl}_{3}\right): \delta 21.0\left(\mathrm{CH}_{3}\right) ; 22.0\left(\mathrm{CH}_{3}\right) ; 24.8$ $\left(\mathrm{CH}_{2}\right) ; 25.8\left(\mathrm{CH}_{3}\right) ; 31.2(\mathrm{CH}) ; 34.8\left(\mathrm{CH}_{2}\right) ; 40.8\left(\mathrm{CH}_{2}\right)$ $41.3\left(\mathrm{CH}_{2}\right) ; 43.4(\mathrm{CH}) ; 56.0(\mathrm{C}) ; 69.7(\mathrm{C}) ; 76.7(\mathrm{CH}) ; 87.7$ $(\mathrm{CH}) ; 113.4\left(\mathrm{CH}_{2}\right) ; 126.6(\mathrm{CH}) ; 127.7(\mathrm{CH}) ; 139.2(\mathrm{CH})$ IR (nujol dispersion): 3320 (broad), 3095, 1655, 830, 730 $\mathrm{cm}^{-1}$. HRMS calcd for $\mathrm{C}_{17} \mathrm{H}_{28} \mathrm{NO}_{2}[\mathrm{M}+\mathrm{H}]^{+} 278.2115$, found 278.2118 .

\section{$(3 R, 4 \mathrm{a} R, 5 \mathrm{a} S, 6 S, 11 \mathrm{a} S)-3,11,11-t$ rimethyl-6-vinyl-} 2,3,4,4a,5a,6,11,11a-octahydro-1H,9H-

benzo[e]pyrido[2,1-b][1,3]oxazin-6-ol (23a). This compound was obtained from 19a $(1.0 \mathrm{~g})$ and catalyst 6 and purified by flash chromatography (hexanes/ ethyl acetate $20: 1)$. Yield: $0.24 \mathrm{~g}, 26 \%$. Colorless oil. $[\alpha]_{\mathrm{D}}{ }^{25}=-$ $113.3\left(\mathrm{c}=1.5, \mathrm{CH}_{2} \mathrm{Cl}_{2}\right)$. ${ }^{\mathrm{H}} \mathrm{NMR}\left(300 \mathrm{MHz}, \mathrm{CDCl}_{3}\right): \delta$ $0.90-1.15(\mathrm{~m}, 3 \mathrm{H}) ; 0.92(\mathrm{~d}, J=6.6 \mathrm{~Hz}, 3 \mathrm{H}) ; 1.16(\mathrm{~s}, 3 \mathrm{H})$; $1.17(\mathrm{~s}, 3 \mathrm{H}) ; 1.56(\mathrm{~m}, 1 \mathrm{H}) ; 1.57-1.73(\mathrm{~m}, 3 \mathrm{H}) ; 1.90(\mathrm{~m}$ $1 \mathrm{H}) ; 3.22(\mathrm{~m}, 1 \mathrm{H}) ; 3.28(\mathrm{~s}, 1 \mathrm{H}) ; 3.40(\mathrm{~m}, 1 \mathrm{H}) ; 3.50\left(\mathrm{td}, J_{l}\right.$ $\left.=10.3 \mathrm{~Hz}, J_{2}=3.7 \mathrm{~Hz}, 1 \mathrm{H}\right) ; 4.55(\mathrm{~s}, 1 \mathrm{H}) ; 5.13\left(\mathrm{dd}, J_{l}=\right.$ $\left.10.6 \mathrm{~Hz}, J_{2}=1.7 \mathrm{~Hz}, 1 \mathrm{H}\right) ; 5.36\left(\mathrm{dd}, J_{l}=17.3 \mathrm{~Hz}, J_{2}=1.7\right.$ $\mathrm{Hz}, 1 \mathrm{H}) ; 5.52(\mathrm{~d}, J=10.0 \mathrm{~Hz}, 1 \mathrm{H}) ; 5.78(\mathrm{~m}, 1 \mathrm{H}) ; 5.93(\mathrm{dd}$, $\left.J_{l}=17.3 \mathrm{~Hz}, J_{2}=10.6 \mathrm{~Hz}, 1 \mathrm{H}\right) .{ }^{13} \mathrm{C} N M R(75 \mathrm{MHz}$ $\left.\mathrm{CDCl}_{3}\right): \delta 20.9\left(\mathrm{CH}_{3}\right) ; 22.0\left(\mathrm{CH}_{3}\right) ; 246\left(\mathrm{CH}_{2}\right) ; 25.7\left(\mathrm{CH}_{3}\right)$ $31.1(\mathrm{CH}) ; 34.7\left(\mathrm{CH}_{2}\right) ; 40.8\left(\mathrm{CH}_{2}\right) ; 41.3\left(\mathrm{CH}_{2}\right) ; 43.1(\mathrm{CH})$ 56.0 (C); $70.9(\mathrm{C}) ; 76.7(\mathrm{CH}) ; 85.7(\mathrm{CH}) ; 113.5\left(\mathrm{CH}_{2}\right)$ 124.8 (HC); 127.1 (HC); 140.7 (HC). IR (film): 3545 (broad), 3040, $725 \mathrm{~cm}^{-1}$. HRMS calcd for $\mathrm{C}_{17} \mathrm{H}_{28} \mathrm{NO}_{2}[\mathrm{M}+$ $\mathrm{H}]^{+} 278.2115$, found 278.2113 .

\section{$(3 R, 4 \mathrm{a} R, 5 \mathrm{a} S, 6 R, 11 \mathrm{a} S)-6-m e t h o x y-3,11,11$-trimethyl-6- vinyl-2,3,4,4a,5a,6,11,11a-octahydro- $1 \mathrm{H}, 9 \mathrm{H}$ -}

benzo[e]pyrido $[2,1-b][1,3]$ oxazine $(24 a)$. This compound was obtained from $\mathbf{2 0 a}(1.0 \mathrm{~g})$ and catalyst $\mathbf{5}$, and purified by flash chromatography (hexanes/ ethyl acetate 20:1). Yield: $0.55 \mathrm{~g}, 60 \%$. Colorless oil. $[\alpha]_{\mathrm{D}}{ }^{25}=-132.3(\mathrm{c}=0.5$ $\left.\mathrm{CH}_{2} \mathrm{Cl}_{2}\right) .{ }^{1} \mathrm{H}$ NMR $\left(300 \mathrm{MHz}, \mathrm{CDCl}_{3}\right): \delta 0.89(\mathrm{~d}, J=6.5$ $\mathrm{Hz}, 3 \mathrm{H}) ; 0.95-1.01(\mathrm{~m}, 2 \mathrm{H}) ; 1.05(\mathrm{~m}, 1 \mathrm{H}) ; 1.21(\mathrm{~s}, 3 \mathrm{H})$ $1.26(\mathrm{~s}, 3 \mathrm{H}) ; 1.42-1.60(\mathrm{~m}, 3 \mathrm{H}) ; 1.69(\mathrm{~m}, 1 \mathrm{H}) ; 1.84(\mathrm{~m}$ $1 \mathrm{H}) ; 3.24(\mathrm{~s}, 3 \mathrm{H}) ; 3.33-3.53(\mathrm{~m}, 3 \mathrm{H}) ; 4.65(\mathrm{~s}, 1 \mathrm{H}) ; 5.28$ (dd, $\left.J_{l}=10.8 \mathrm{~Hz}, J_{2}=1.2 \mathrm{~Hz}, 1 \mathrm{H}\right) ; 5.30\left(\mathrm{dd}, J_{l}=17.8 \mathrm{~Hz}, J_{2}=\right.$ $1.2 \mathrm{~Hz}, 1 \mathrm{H}) ; 5.82(\mathrm{~m}, 1 \mathrm{H}) ; 5.83\left(\mathrm{dd}, J_{l}=17.8 \mathrm{~Hz}, J_{2}=10.8\right.$ $\mathrm{Hz}, 1 \mathrm{H}), 6.10(\mathrm{~m}, 1 \mathrm{H}) .{ }^{13} \mathrm{C}$ NMR $\left(75 \mathrm{MHz}, \mathrm{CDCl}_{3}\right): \delta 21.0$ $\left(\mathrm{CH}_{3}\right) ; 22.1\left(\mathrm{CH}_{3}\right) ; 25.0\left(\mathrm{CH}_{2}\right) ; 26.0\left(\mathrm{CH}_{3}\right) ; 31.4(\mathrm{CH})$ $35.0\left(\mathrm{CH}_{2}\right) ; 41.1\left(\mathrm{CH}_{2}\right) ; 41.9\left(\mathrm{~N}-\mathrm{CH}_{2}\right) ; 43.5(\mathrm{CH}) ; 50.8$ 
$\left(\mathrm{CH}_{3}\right) ; 56.0(\mathrm{C}) ; 75.1(\mathrm{C}) ; 76.9(\mathrm{CH}) ; 86.7(\mathrm{CH}) ; 115.5$ $\left(\mathrm{CH}_{2}\right) ; 121.5$ (HC); 131.0 (HC); 138.7 (HC). IR (film): $3085,1650,81,730 \mathrm{~cm}^{-1}$. HRMS calcd for $\mathrm{C}_{18} \mathrm{H}_{30} \mathrm{NO}_{2}[\mathrm{M}$ $+\mathrm{H}]^{+}$292.2271, found 292.2273

\section{$(3 R, 4 a R, 5 a S, 6 S, 11 a S)-6-m e t h o x y-3,11,11-t r i m e t h y l-6-$} vinyl-2,3,4,4a,5a,6,11,11a-octahydro-1 $H, 9 H$

benzo $[e]$ pyrido $[2,1-b][1,3]$ oxazine $(25 a)$. This compound was obtained from $20 \mathrm{a}(1.0 \mathrm{~g})$ and catalyst $\mathbf{6}$, and purified by flash chromatography (hexanes/ ethyl acetate 20:1). Yield: $0.58 \mathrm{~g}, 64 \%$. White solid. $\mathrm{Mp} 77-79{ }^{\circ} \mathrm{C}$ (from pentane $) .[\alpha]_{\mathrm{D}}^{25}=-129.9\left(\mathrm{c}=1.0, \mathrm{CH}_{2} \mathrm{Cl}_{2}\right) .{ }^{1} \mathrm{H}$ NMR $(300$ $\left.\mathrm{MHz}_{\mathrm{CDCl}}\right)_{3}: \delta 0.86-1.02(\mathrm{~m}, 2 \mathrm{H}) ; 0.91(\mathrm{~d}, J=6.5 \mathrm{~Hz}$, $3 \mathrm{H}) ; 1.13(\mathrm{~m}, 1 \mathrm{H}) ; 1.15(\mathrm{~s}, 3 \mathrm{H}) ; 1.20(\mathrm{~s}, 3 \mathrm{H}) ; 1.50(\mathrm{~m}$, $1 \mathrm{H}) ; 1.54-1.72(\mathrm{~m}, 2 \mathrm{H}) ; 1.98(\mathrm{~m}, 1 \mathrm{H}) ; 3.22(\mathrm{~m}, 1 \mathrm{H}) ; 3.32$ $(\mathrm{s}, 3 \mathrm{H}) ; 3.43-3.51(\mathrm{~m}, 2 \mathrm{H}) ; 4.75(\mathrm{~s}, 1 \mathrm{H}) ; 5.23\left(\mathrm{dd}, J_{l}=10.7\right.$ $\left.\mathrm{Hz}, J_{2}=1.4 \mathrm{~Hz}, 1 \mathrm{H}\right), 5.28\left(\mathrm{dd}, J_{l}=17.7 \mathrm{~Hz}, J_{2}=1.4 \mathrm{~Hz}\right.$, $1 \mathrm{H}) ; 5.60(\mathrm{~d}, J=10.2 \mathrm{~Hz}, 1 \mathrm{H}) ; 5.81(\mathrm{~m}, 1 \mathrm{H}) ; 5.85\left(\mathrm{dd}, J_{l}=\right.$ $\left.17.7 \mathrm{~Hz}, J_{2}=10.7 \mathrm{~Hz}, 1 \mathrm{H}\right) .{ }^{3} \mathrm{C}$ NMR $\left(75 \mathrm{MHz}, \mathrm{CDCl}_{3}\right): \delta$ $21.5\left(\mathrm{CH}_{3}\right) ; 22.2\left(\mathrm{CH}_{3}\right) ; 25.0\left(\mathrm{CH}_{2}\right) ; 26.1\left(\mathrm{CH}_{3}\right) ; 31.5$ (CH); $35.0\left(\mathrm{CH}_{2}\right) ; 41.3\left(\mathrm{CH}_{2}\right) ; 41.9\left(\mathrm{CH}_{2}\right) ; 43.1(\mathrm{CH}) ; 50.6$ $\left(\mathrm{CH}_{3}\right) ; 56.3(\mathrm{C}) ; 76.5(\mathrm{C}) 77.1(\mathrm{CH}) ; 84.5(\mathrm{CH}) ; 115.8$ $\left(\mathrm{CH}_{2}\right) ; 124.0(\mathrm{CH}) ; 126.1(\mathrm{CH}) ; 139.7(\mathrm{CH})$. IR (film): $3085,1664,723,656 \mathrm{~cm}^{-1}$. HRMS calcd for $\mathrm{C}_{18} \mathrm{H}_{30} \mathrm{NO}_{2}[\mathrm{M}$ $+\mathrm{H}]^{+}$292.2271, found 292.2271

$(3 R, 4 \mathrm{a} R, 5 \mathrm{a} S, 6 R, 11 \mathrm{a} S)-6-$ methoxy-3,7,11,11tetramethyl-6-(prop-1-en-2-yl)-2,3,4,4a,5a,6,11,11aoctahydro-1 $H, 9 H$-benzo[e]pyrido $[2,1-b][1,3]$ oxazine

(24b). This compound was obtained from $20 \mathrm{~b}(1.0 \mathrm{~g})$ and catalyst $\mathbf{6}$, and purified by flash chromatography in $\mathrm{CH}_{2} \mathrm{Cl}_{2}$. Yield: $0.69 \mathrm{~g}, 75 \%$. Colorless oil. $[\alpha]_{\mathrm{D}}{ }^{25}=-76.4(\mathrm{c}=1.0$, $\mathrm{CH}_{2} \mathrm{Cl}_{2}$ ). $\mathrm{H}$ NMR (300 MHz, $\left.\mathrm{CDCl}_{3}\right): \delta 0.89(\mathrm{~d}, J=6.5$ $\mathrm{Hz}, 3 \mathrm{H}) ; 0.91-1.13(\mathrm{~m}, 3 \mathrm{H}) ; 1.12(\mathrm{~s}, 3 \mathrm{H}) ; 1.14(\mathrm{~s}, 3 \mathrm{H})$; $1.19-1.47(\mathrm{~m}, 2 \mathrm{H}) ; 1.55(\mathrm{~d}, J=1.5 \mathrm{~Hz}, 3 \mathrm{H}) ; 1.56-1.75(\mathrm{~m}$, $2 \mathrm{H}) ; 1.76(\mathrm{~s}, 3 \mathrm{H}) ; 1.89(\mathrm{~m}, 1 \mathrm{H}) ; 3.16(\mathrm{~m}, 1 \mathrm{H}) ; 3.30(\mathrm{~s}$, $3 \mathrm{H}) ; 3.36\left(\mathrm{td}, J_{l}=10.4 \mathrm{~Hz}, J_{2}=4.0 \mathrm{~Hz}, 1 \mathrm{H}\right) ; 3.45(\mathrm{~m}, 1 \mathrm{H})$; $4.59(\mathrm{~s}, 1 \mathrm{H}) ; 4.93(\mathrm{~d}, J=1.2 \mathrm{~Hz}, 1 \mathrm{H}) ; 5.15(\mathrm{~m}, 1 \mathrm{H}$, $\mathrm{CHH}=) ; 5.87(\mathrm{~d}, J=1.2 \mathrm{~Hz}, 1 \mathrm{H}) \cdot{ }^{13} \mathrm{C}$ NMR $(75 \mathrm{MHz}$, $\left.\mathrm{CDCl}_{3}\right): \delta 17.8\left(\mathrm{CH}_{3}\right) ; 18.4\left(\mathrm{CH}_{3}\right) ; 20.9\left(\mathrm{CH}_{3}\right) ; 22.1\left(\mathrm{CH}_{3}\right) ;$ $24.8\left(\mathrm{CH}_{2}\right) ; 26.4\left(\mathrm{CH}_{3}\right) ; 31.1(\mathrm{CH}) ; 34.8\left(\mathrm{CH}_{2}\right) ; 40.9$ $\left(2 \mathrm{CH}_{2}\right) ; 44.9(\mathrm{CH}) ; 51.9\left(\mathrm{CH}_{3}\right) ; 55.5(\mathrm{C}) ; 75.6(\mathrm{CH}) ; 81.7$ (C); $87.4(\mathrm{CH}) ; 111.6\left(\mathrm{CH}_{2}\right) ; 127.1(\mathrm{CH}) ; 139.2(\mathrm{C}) ; 145.9$ (C). IR (film): $3085,1675,810,735,645 \mathrm{~cm}^{-1}$. HRMS calcd for $\mathrm{C}_{20} \mathrm{H}_{34} \mathrm{NO}_{2}[\mathrm{M}+\mathrm{H}]^{+} 320,2584$, found 320.2587

\section{$(3 R, 4 \mathrm{a} R, 5 \mathrm{a} S, 6 R, 12 \mathrm{a} S)-3,8,12,12-$ tetramethyl-6-(2-} methylallyl)-2,3,4,4a,5a,6,7,10,12,12a-decahydro- $1 H$ benzo[5,6][1,3]oxazino[3,2-a]azepin-6-ol (22c). This compound was obtained from 19c $(1.0 \mathrm{~g})$ and catalyst 5 , and purified by flash chromatography (hexanes/ ethyl acetate $35: 1$ ). Yield: $0.66 \mathrm{~g}, 72 \%$. Colorless oil. $[\alpha]_{\mathrm{D}}{ }^{25}=-$ $38.1\left(\mathrm{c}=1.0, \mathrm{CH}_{2} \mathrm{Cl}_{2}\right) .{ }^{1} \mathrm{H} \mathrm{NMR}\left(400 \mathrm{MHz}, \mathrm{CDCl}_{3}\right): \delta$ $0.84-0.93(\mathrm{~m}, 2 \mathrm{H}) ; 0.92(\mathrm{~d}, J=6.5 \mathrm{~Hz}, 3 \mathrm{H}) ; 1.09(\mathrm{~m}, 1 \mathrm{H})$; $1.14(\mathrm{~s}, 3 \mathrm{H}) ; 1.16(\mathrm{~s}, 3 \mathrm{H}) ; 1.38(\mathrm{~m}, 1 \mathrm{H}) ; 1.43(\mathrm{~m}, 1 \mathrm{H}) ; 1.51$ $(\mathrm{m}, 1 \mathrm{H}) ; 1.66(\mathrm{~m}, 1 \mathrm{H}) ; 1.70(\mathrm{~s}, 3 \mathrm{H}) ; 1.76(\mathrm{~m}, 1 \mathrm{H}) ; 1.85(\mathrm{~s}$, $3 \mathrm{H}) ; 2.14(\mathrm{~d}, J=13.5 \mathrm{~Hz}, 1 \mathrm{H}) ; 2.35(\mathrm{~d}, J=13.5 \mathrm{~Hz}, 1 \mathrm{H})$; $2.78-2.91(\mathrm{~m}, 3 \mathrm{H}) ; 3.36\left(\mathrm{td}, J_{l}=10.7 \mathrm{~Hz}, J_{2}=3.9 \mathrm{~Hz}, 1 \mathrm{H}\right)$; $3.62\left(\mathrm{dd}, J_{l}=13.5 \mathrm{~Hz}, J_{2}=6.4 \mathrm{~Hz}, 1 \mathrm{H}\right) ; 4.48(\mathrm{~s}, 1 \mathrm{H}) ; 4.74$ $(\mathrm{m}, 1 \mathrm{H}) ; 4.89(\mathrm{~m}, 1 \mathrm{H}) ; 5.64(\mathrm{~m}, 1 \mathrm{H}) .{ }^{3} \mathrm{C}$ NMR $(75 \mathrm{MHz}$, $\left.\mathrm{CDCl}_{3}\right): \delta 22.2\left(2 \mathrm{CH}_{3}\right) ; 24.5\left(\mathrm{CH}_{3}\right) ; 25.1\left(\mathrm{CH}_{2}\right) ; 26.2$ $\left(\mathrm{CH}_{3}\right) ; 28.6\left(\mathrm{CH}_{3}\right) ; 31.4(\mathrm{CH}) ; 35.0\left(\mathrm{CH}_{2}\right) ; 37.4\left(\mathrm{CH}_{2}\right)$; $39.2\left(\mathrm{CH}_{2}\right) ; 41.1\left(\mathrm{CH}_{2}\right) ; 45.8(\mathrm{CH}) ; 46.9\left(\mathrm{CH}_{2}\right) ; 57.8(\mathrm{C})$; $70.7(\mathrm{C}) ; 76.7(\mathrm{CH}) ; 89.4(\mathrm{CH}) ; 114.4\left(\mathrm{CH}_{2}\right) ; 124.6(\mathrm{CH})$; 139.5 (C); 143.3 (C). $\delta$ IR (film): 3080, 1640, 740, 670 $\mathrm{cm}^{-1}$. HRMS calcd for $\mathrm{C}_{21} \mathrm{H}_{36} \mathrm{NO}_{2}[\mathrm{M}+\mathrm{H}]^{+} 334.2741$, found 334.2741 .

$(3 R, 4 \mathrm{a} R, 5 \mathrm{a} S, 6 S, 12 \mathrm{a} S)-3,8,12,12-t$ tramethyl-6-(2methylallyl)-2,3,4,4a,5a,6,7,10,12,12a-decahydro- $1 H$ benzo $[5,6][1,3]$ oxazino[3,2-a]azepin-6-ol (23c). This compound was obtained from 19c $(1.0 \mathrm{~g})$ and catalyst $\mathbf{6}$, and purified by flash chromatography (hexanes/ ethyl acetate 35:1). Yield: $0.18 \mathrm{~g}, 20 \%$. Colorless oil. $[\alpha]_{\mathrm{D}}{ }^{25}=-$ $55.2\left(\mathrm{c}=1.0, \mathrm{CH}_{2} \mathrm{Cl}_{2}\right)$. ${ }^{1} \mathrm{H}$ NMR $\left(300 \mathrm{MHz}, \mathrm{CDCl}_{3}\right): \delta$ $0.84-1.02(\mathrm{~m}, 1 \mathrm{H}) ; 0.93(\mathrm{~d}, J=6.4 \mathrm{~Hz}, 3 \mathrm{H}) ; 1.07(\mathrm{~m}, 1 \mathrm{H})$; $1.16(\mathrm{~s}, 3 \mathrm{H}) ; 1.16(\mathrm{~s}, 3 \mathrm{H}) ; 1.30(\mathrm{~m}, 1 \mathrm{H}) ; 1.48(\mathrm{~m}, 1 \mathrm{H}) ;$ $1.54(\mathrm{~m}, 1 \mathrm{H}) ; 1.66(\mathrm{~m}, 1 \mathrm{H}) ; 1.73(\mathrm{~s}, 3 \mathrm{H}) ; 1.85(\mathrm{~s}, 3 \mathrm{H}) ; 1.91$ $(\mathrm{m}, 1 \mathrm{H}) ; 2.23(\mathrm{~d}, J=15.5 \mathrm{~Hz}, 1 \mathrm{H}) ; 2.25(\mathrm{~d}, J=13.5 \mathrm{~Hz}$,
$1 \mathrm{H}) ; 2.39(\mathrm{~d}, J=13.5 \mathrm{~Hz}, 1 \mathrm{H}) ; 2.50(\mathrm{~d}, J=15.5 \mathrm{~Hz}, 1 \mathrm{H})$ $2.98\left(\mathrm{dd}, J_{l}=15.2 \mathrm{~Hz}, J_{2}=7.5 \mathrm{~Hz}, 1 \mathrm{H}\right) ; 3.07(\mathrm{~s}$ broad, $1 \mathrm{H}) ; 3.49\left(\mathrm{td}, J_{l}=10.5 \mathrm{~Hz}, J_{2}=4.0 \mathrm{~Hz}, 1 \mathrm{H}\right) ; 3.54(\mathrm{~m}, 1 \mathrm{H})$; $4.57(\mathrm{~s}, 1 \mathrm{H}) ; 4.76(\mathrm{~d}, J=0.9 \mathrm{~Hz}, 1 \mathrm{H}) ; 4.87(\mathrm{~d}, J=0.9 \mathrm{~Hz}$ $1 \mathrm{H}) ; \quad 5.50(\mathrm{~m}, 1 \mathrm{H}) \cdot{ }^{13} \mathrm{C}$ NMR $\left(75 \mathrm{MHz}, \mathrm{CDCl}_{3}\right): \delta 20.7$ $\left(\mathrm{CH}_{3}\right) ; 22.2\left(\mathrm{CH}_{3}\right) ; 24.8\left(\mathrm{CH}_{3}\right) ; 25.0\left(\mathrm{CH}_{2}\right) ; 26.1\left(\mathrm{CH}_{3}\right)$; $28.1\left(\mathrm{CH}_{3}\right) ; 31.3(\mathrm{CH}) ; 34.9\left(\mathrm{CH}_{2}\right) ; 39.1\left(\mathrm{CH}_{2}\right) ; 40.6$ $\left(\mathrm{CH}_{2}\right) ; 41.1\left(\mathrm{CH}_{2}\right) ; 46.1(\mathrm{CH}) ; 47.3\left(\mathrm{CH}_{2}\right) ; 57.3(\mathrm{C}) ; 70.4$ (C); 76.1 (CH); 89.2 (CH); $114.7\left(\mathrm{CH}_{2}\right) ; 124.2(\mathrm{CH}) ; 136.1$ (C); 143.0 (C). IR (film): $3545,3070,1640,740,630 \mathrm{~cm}^{-1}$ HRMS calcd for $\mathrm{C}_{21} \mathrm{H}_{36} \mathrm{NO}_{2}[\mathrm{M}+\mathrm{H}]^{+} 334.2741$, found 334.2743

\section{$(3 R, 4 \mathrm{a} R, 5 \mathrm{a} S, 6 R, 12 \mathrm{a} S)-6-m e t h o x y-3,8,12,12$}

tetramethyl-6-(2-methylallyl)-2,3,4,4a,5a,6,7,10,12,12adecahydro- $1 H$-benzo $[5,6][1,3]$ oxazino $[3,2$-a $]$ azepine

$(\mathbf{2 4 c})$. This compound was obtained from $20 \mathrm{c}(1.0 \mathrm{~g})$ and catalyst $\mathbf{5}$, and purified by flash chromatography (hexanes/ ethyl acetate 30:1). Yield: $0.87 \mathrm{~g}, 94 \%$. White solid. Mp $59-61{ }^{\circ} \mathrm{C}$ (from pentane) $[\alpha]_{\mathrm{D}}{ }^{25}=-38.9\left(\mathrm{c}=1.0, \mathrm{CH}_{2} \mathrm{Cl}_{2}\right)$ ${ }^{1} \mathrm{H}$ NMR $\left(300 \mathrm{MHz}, \mathrm{CDCl}_{3}\right): \delta 0.84-0.99(\mathrm{~m}, 2 \mathrm{H}) ; 0.91(\mathrm{~d}$ $J=6.5 \mathrm{~Hz}, 3 \mathrm{H}) ; 1.02(\mathrm{~m}, 1 \mathrm{H}) ; 1.08(\mathrm{~s}, 3 \mathrm{H}) ; 1.16(\mathrm{~s}, 3 \mathrm{H})$ $1.31(\mathrm{~m}, 1 \mathrm{H}) ; 1.43(\mathrm{~m}, 1 \mathrm{H}) ; 1.54(\mathrm{~m}, 1 \mathrm{H}) ; 1.68(\mathrm{~m}, 1 \mathrm{H})$ $1.74(\mathrm{~s}, 3 \mathrm{H}) ; 1.83(\mathrm{~s}, 3 \mathrm{H}) ; 1.87(\mathrm{~m}, 1 \mathrm{H}) ; 2.06(\mathrm{~d}, J=16.1$ $\mathrm{Hz}, 1 \mathrm{H}) ; 2.28(\mathrm{~d}, J=14.8 \mathrm{~Hz}, 1 \mathrm{H}) ; 2.42(\mathrm{~d}, J=14.8 \mathrm{~Hz}$, $1 \mathrm{H}) ; 2.56(\mathrm{~d}, J=16.1 \mathrm{~Hz}, 1 \mathrm{H}) ; 3.15\left(\mathrm{dd}, J_{1}=15.6 \mathrm{~Hz}, J_{2}=\right.$ $5.6 \mathrm{~Hz}, 1 \mathrm{H}) ; 3.22(\mathrm{~s}, 3 \mathrm{H}) ; 3.33\left(\mathrm{td}, J_{l}=10.6 \mathrm{~Hz}, J_{2}=4.0\right.$ $\mathrm{Hz}, 1 \mathrm{H}) ; 3.40(\mathrm{~m}, 1 \mathrm{H}) ; 4.43(\mathrm{~s}, 1 \mathrm{H}) ; 4.81(\mathrm{~d}, J=0.9 \mathrm{~Hz}$ $1 \mathrm{H}) ; 4.86 \mathrm{~d}, J=0.9 \mathrm{~Hz}, 1 \mathrm{H}) ; 5.49(\mathrm{~m}, 1 \mathrm{H}) .{ }^{13} \mathrm{C}$ NMR $(75$ $\left.\mathrm{MHz}, \mathrm{CDCl}_{3}\right): \delta 17.7\left(\mathrm{CH}_{3}\right) ; 21.9\left(\mathrm{CH}_{3}\right) ; 24.0\left(\mathrm{CH}_{3}\right) ; 24.8$ $\left(\mathrm{CH}_{2}\right) ; 25.5\left(\mathrm{CH}_{3}\right) ; 27.3\left(\mathrm{CH}_{3}\right) ; 30.8(\mathrm{CH}) ; 34.6\left(\mathrm{CH}_{2}\right)$; $37.4\left(\mathrm{CH}_{2}\right) ; 38.6\left(\mathrm{CH}_{2}\right) ; 39.2\left(\mathrm{CH}_{2}\right) ; 40.8\left(\mathrm{CH}_{2}\right) ; 47.1$ $(\mathrm{CH}) ; 49.3\left(\mathrm{CH}_{3}\right) ; 57.0(\mathrm{C}) ; 75.4(\mathrm{CH}) ; 80.6(\mathrm{C}) ; 88.9$ $(\mathrm{CH}) ; 113.6\left(\mathrm{CH}_{2}\right) ; 124.6(\mathrm{CH}) ; 134.1(\mathrm{C}) ; 142.1$ (C). IR (nujol dispersion): $3075,1640,1455,890 \mathrm{~cm}^{-1}$. . HRMS calcd for $\mathrm{C}_{22} \mathrm{H}_{38} \mathrm{NO}_{2}[\mathrm{M}+\mathrm{H}]^{+}$348.2897, found 348.2906.

$(3 R, 4 \mathrm{a} R, 5 \mathrm{a} S, 6 S, 12 \mathrm{a} S)-6-(($ tert-butyldimethylsilyl)oxy)$3,8,12,12$-tetramethyl-6-(2-methylallyl)$\mathbf{2 , 3 , 4 , 4 a , 5 a , 6 , 7 , 1 0 , 1 2 , 1 2 a - d e c a h y d r o - 1 H}$ benzo $[5,6][1,3]$ oxazino $[3,2-a]$ azepine

(27c). This compound was obtained from 21c $(0.6 \mathrm{~g})$ and catalyst 6 , and purified by flash chromatography (hexanes/ ethyl acetate $80: 1)$. Yield: $0.48 \mathrm{~g}, 85 \%$. Colorless oil $[\alpha]_{\mathrm{D}}{ }^{25}=-$ $81.5\left(\mathrm{c}=1.0, \mathrm{CH}_{2} \mathrm{Cl}_{2}\right) .{ }^{\mathrm{l}} \mathrm{H}$ NMR $\left(300 \mathrm{MHz}, \mathrm{CDCl}_{3}\right): \delta 0.07$ $(\mathrm{s}, 3 \mathrm{H}) ; 0.78(\mathrm{~s}, 3 \mathrm{H}) ; 0.85(\mathrm{~s}, 9 \mathrm{H}) ; 0.86-0.98(\mathrm{~m}, 2 \mathrm{H}) ; 0.93$ $(\mathrm{d}, J=6.6 \mathrm{~Hz}, 3 \mathrm{H}) ; 1.11(\mathrm{~m}, 1 \mathrm{H}) ; 1.13(\mathrm{~s}, 3 \mathrm{H}) ; 1.15(\mathrm{~s}$, $3 \mathrm{H}) ; 1.35(\mathrm{~m}, 1 \mathrm{H}) ; 1.42-1.52(\mathrm{~m}, 2 \mathrm{H}) ; 1.61(\mathrm{~m}, 1 \mathrm{H}) ; 1.71$ $(\mathrm{s}, 3 \mathrm{H}) ; 1.82(\mathrm{~s}, 3 \mathrm{H}) ; 1.93-2.00(\mathrm{~m}, 2 \mathrm{H}) ; 2.31(\mathrm{~d}, J=13.2$ $\mathrm{Hz}, 1 \mathrm{H}) ; 2.46(\mathrm{~d}, J=13.2 \mathrm{~Hz}, 1 \mathrm{H}) ; 2.76(\mathrm{~d}, J=14.8 \mathrm{~Hz}$, $1 \mathrm{H}) ; 2.93\left(\mathrm{dd}, J_{l}=14.5 \mathrm{~Hz}, J_{2}=8.2 \mathrm{~Hz}, 1 \mathrm{H}\right) ; 3.45\left(\mathrm{td}, J_{l}=\right.$ $\left.10.6 \mathrm{~Hz}, J_{2}=3.9 \mathrm{~Hz}, 1 \mathrm{H}\right) ; 3.69(\mathrm{~m}, 1 \mathrm{H}) ; 4.71(\mathrm{~s}, 1 \mathrm{H}) ; 4.82$ $(\mathrm{m}, 1 \mathrm{H}) ; 4.84(\mathrm{~m}, 1 \mathrm{H}) ; 5.44(\mathrm{~m}, 1 \mathrm{H}) .{ }^{3} \mathrm{C}$ NMR $(75 \mathrm{MHz}$ $\left.\mathrm{CDCl}_{3}\right): \delta-2.5\left(\mathrm{CH}_{3}\right) ;-1.6\left(\mathrm{CH}_{3}\right) ; 18.8(\mathrm{C}) ; 22.9\left(\mathrm{CH}_{3}\right)$ $22.4\left(\mathrm{CH}_{3}\right) ; 25.2\left(\mathrm{CH}_{2}+\mathrm{CH}_{3}\right) ; 26.3\left(3 \mathrm{CH}_{3}\right) ; 26.4\left(\mathrm{CH}_{3}\right)$ $28.7\left(\mathrm{CH}_{3}\right) ; 31.4(\mathrm{CH}) ; 35.1\left(\mathrm{CH}_{2}\right) ; 38.3\left(\mathrm{CH}_{2}\right) ; 41.1$ $\left(\mathrm{CH}_{2}\right) ; 42.2\left(\mathrm{CH}_{2}\right) ; 45.4(\mathrm{CH}) ; 47.1\left(\mathrm{CH}_{2}\right) 57.5(\mathrm{C}) ; 76.4$ (O); $77.8(\mathrm{C}) ; 88.6(\mathrm{CH}) ; 115.3\left(\mathrm{CH}_{2}\right) ; 124.4(\mathrm{CH}) ; 136.9$ (C); 142.7 (C IR (film): 3075, 1640, 705, $620 \mathrm{~cm}^{-1}$ HRMS calcd for $\mathrm{C}_{27} \mathrm{H}_{50} \mathrm{NO}_{2} \mathrm{Si}[\mathrm{M}+\mathrm{H}]^{+}$448.3605, found 448.3600

\section{$(3 R, 4 \mathrm{a} R, 5 \mathrm{a} S, 6 R, 12 \mathrm{a} S)-6$-methoxy-3,12,12-trimethyl-6-} vinyl-2,3,4,4a,5a,6,9,10,12,12a-decahydro- $1 H$ benzo[5,6][1,3] oxazino[3,2-a]azepine (24d). This compound was obtained from $20 \mathrm{~d}(1.0 \mathrm{~g})$ and catalyst $\mathbf{5}$ and purified by flash chromatography (hexanes/ ethyl acetate 25:1). Yield: $0.37 \mathrm{~g}, 40 \%$. Colorless oil. $[\alpha]^{25}=-$ $75.2\left(\mathrm{c}=1.0, \mathrm{CH}_{2} \mathrm{Cl}_{2}\right) .{ }^{1} \mathrm{H}$ NMR $\left(400 \mathrm{MHz}, \mathrm{CDCl}_{3}\right): \delta$ $0.78-0.93(\mathrm{~m}, 2 \mathrm{H}) ; 0.85(\mathrm{~d}, J=6.6 \mathrm{~Hz}, 3 \mathrm{H}) ; 1.03(\mathrm{~m}, 1 \mathrm{H})$ $1.08(\mathrm{~m}, 3 \mathrm{H}) ; 1.12(\mathrm{~s}, 3 \mathrm{H}) ; 1.29(\mathrm{~m}, 1 \mathrm{H}) ; 1.39(\mathrm{~m}, 1 \mathrm{H})$; $1.55(\mathrm{~m}, 1 \mathrm{H}) ; 1.62(\mathrm{~m}, 1 \mathrm{H}) ; 1.85(\mathrm{~m}, 1 \mathrm{H}) ; 2.13(\mathrm{~m}, 1 \mathrm{H})$; $2.23(\mathrm{~m}, 1 \mathrm{H}) ; 2.92\left(\mathrm{ddd}, 1 \mathrm{H}, J_{l}=14.1 \mathrm{~Hz}, J_{2}=7.4 \mathrm{~Hz}, J_{3}=\right.$ $4.2 \mathrm{~Hz}, 1 \mathrm{H}) ; 3.07(\mathrm{~m}, 1 \mathrm{H}) ; 3.11(\mathrm{~s}, 3 \mathrm{H}) ; 3.34\left(\mathrm{td}, J_{l}=10.6\right.$ $\left.\mathrm{Hz}, J_{2}=4.1 \mathrm{~Hz}\right) ; 4.51(\mathrm{~s}, 1 \mathrm{H}) ; 5.20\left(\mathrm{dd}, J_{l}=10.7 \mathrm{~Hz}, J_{2}=\right.$ $1.6 \mathrm{~Hz}, 1 \mathrm{H}) ; 5.22\left(\mathrm{dd}, J_{l}=17.5 \mathrm{~Hz}, J_{2}=1.6 \mathrm{~Hz}, 1 \mathrm{H}\right) ; 5.45$ $\left(\mathrm{dt}, 1 \mathrm{H}, J_{l}=11.5 \mathrm{~Hz}, J_{2}=1.6 \mathrm{~Hz}, 1 \mathrm{H}\right) ; 5.94(\mathrm{~m}, 1 \mathrm{H}) ; 5.95$ (dd, $\left.J_{l}=17.5 \mathrm{~Hz}, J_{2}=10.7 \mathrm{~Hz}, 1 \mathrm{H}\right){ }^{13} \mathrm{C}$ NMR $(100 \mathrm{MHz}$, $\left.\mathrm{CDCl}_{3}\right): \delta 18.4\left(\mathrm{CH}_{3}\right) ; 22.2\left(\mathrm{CH}_{3}\right) ; 27.2\left(\mathrm{CH}_{3}\right) ; 31.3(\mathrm{CH})$ $32.3\left(\mathrm{CH}_{2}\right) ; 34.9\left(\mathrm{CH}_{2}\right) ; 38.3\left(\mathrm{CH}_{2}\right) ; 41.2\left(\mathrm{CH}_{2}\right) ; 48.0$ 
$(\mathrm{CH}) ; 50.3(\mathrm{CH}) ; 57.1(\mathrm{C}) ; 76.5(\mathrm{CH}) ; 81.2(\mathrm{C}) ; 92.3(\mathrm{CH})$ 114.6 $\left(\mathrm{CH}_{2}\right) ; 140.4(\mathrm{CH})$. IR (film): $3075,1740,1455,725$ $\mathrm{cm}^{-1}$. HRMS calcd for $\mathrm{C}_{19} \mathrm{H}_{32} \mathrm{NO}_{2}[\mathrm{M}+\mathrm{H}]^{+}$306.2428, found 306.2428 .

$(3 R, 4 \mathrm{a} R, 5 \mathrm{a} S, 6 S, 12 \mathrm{a} S)-6-m e t h o x y-3,12,12-t r i m e t h y l-6-$ vinyl-2,3,4,4a,5a,6,9,10,12,12a-decahydro-1Hbenzo[5,6][1,3] oxazino[3,2-a]azepine (25d). This compound was obtained from $20 \mathrm{~d}(1.0 \mathrm{~g})$ and catalyst 6 , and purified by flash chromatography (hexanes/ ethyl acetate $25: 1)$. Yield: $0.57 \mathrm{~g}, 62 \%$. Colorless oil. $[\alpha]_{\mathrm{D}}{ }^{25}=-$ $92.8\left(\mathrm{c}=1.5, \mathrm{CHCl}_{3}\right)$. ${ }^{1} \mathrm{H}$ NMR $\left(300 \mathrm{MHz}, \mathrm{CDCl}_{3}\right): \delta$ $0.81-0.98(\mathrm{~m}, 2 \mathrm{H}) ; 0.89(\mathrm{~d}, J=6.5 \mathrm{~Hz}, 3 \mathrm{H}) ; 1.09(\mathrm{~m}, 1 \mathrm{H})$; $1.16(\mathrm{~s}, 3 \mathrm{H}) ; 1.23(\mathrm{~s}, 3 \mathrm{H}) ; 1.40-1.51(\mathrm{~m}, 2 \mathrm{H}) ; 1.54(\mathrm{~m}$, $1 \mathrm{H}) ; 1.66(\mathrm{~m}, 1 \mathrm{H}) ; 2.00(\mathrm{~m}, 1 \mathrm{H}) ; 2.02-2.26(\mathrm{~m}, 2 \mathrm{H}) ; 2.93$ (ddd, $\left.J_{1}=13.6 \mathrm{~Hz}, J_{2}=7.5 \mathrm{~Hz}, J_{3}=2.7 \mathrm{~Hz}, 1 \mathrm{H}\right) ; 3.19(\mathrm{~m}$, $1 \mathrm{H}) ; 3.24(\mathrm{~s}, 3 \mathrm{H}) ; 3.54\left(\mathrm{td}, J_{1}=10.5 \mathrm{~Hz}, J_{2}=3.9 \mathrm{~Hz}, 1 \mathrm{H}\right)$; $4.78(\mathrm{~s}, 1 \mathrm{H}) ; 5.32\left(\mathrm{dd}, J_{1}=10.5 \mathrm{HZ}, J_{2}=1.7 \mathrm{~Hz}, 1 \mathrm{H}\right) ; 5.35$ (dd, $\left.J_{l}=17.5 \mathrm{HZ}, J_{2}=1.7 \mathrm{~Hz}, 1 \mathrm{H}\right) ; 5.66(\mathrm{~d}, J=11.3 \mathrm{~Hz}$, $1 \mathrm{H}) ; 5.82\left(\mathrm{dd}, J_{l}=17.5 \mathrm{~Hz}, J_{2}=10.5 \mathrm{~Hz}, 1 \mathrm{H}\right) ; 6.0\left(\mathrm{dt}, J_{1}=\right.$ $\left.11.3 \mathrm{HZ}, J_{2}=5.5 \mathrm{~Hz}, 1 \mathrm{H}\right) .{ }^{13} \mathrm{C} \mathrm{NMR}\left(75 \mathrm{MHz}, \mathrm{CDCl}_{3}\right): \delta$ $22.2\left(\mathrm{CH}_{3}\right) ; 22.2\left(\mathrm{CH}_{3}\right) ; 25.4\left(\mathrm{CH}_{2}\right) ; 28.1\left(\mathrm{CH}_{3}\right) ; 29.9$ $\left(\mathrm{CH}_{2}\right) ; 31.3(\mathrm{CH}) ; 35.0\left(\mathrm{CH}_{2}\right) ; 40.8\left(\mathrm{CH}_{2}\right) ; 41.5\left(\mathrm{CH}_{2}\right)$; $46.9(\mathrm{CH}) ; 50.8\left(\mathrm{CH}_{3}\right) ; 57.7(\mathrm{C}) ; 77.1(\mathrm{O}) ; 82.1(\mathrm{C}) ; 90.1$ $(\mathrm{CH}) ; 116.0\left(\mathrm{CH}_{2}\right) ; 127.8(\mathrm{CH}) ; 132.4(\mathrm{CH}) ; 137.7(\mathrm{CH})$. IR (film): $3080,1660,730 \mathrm{~cm}^{-1}$. HRMS calcd for $\mathrm{C}_{19} \mathrm{H}_{32} \mathrm{NO}_{2}[\mathrm{M}+\mathrm{H}]^{+}$306.2428, found 306.2431.

$(3 R, 4 \mathrm{a} R, 5 \mathrm{a} S, 6 S, 12 \mathrm{a} S)-6-((t e r t-b u t y l d i m e t h y l s i l y l) o x y)-$ 3,12,12-trimethyl-6-vinyl-2,3,4,4a,5a,6,9,10,12,12adecahydro- $1 H$-benzo $[5,6][1,3]$ oxazino $[3,2-a]$ azepine (27d). This compound was obtained from 21d $(0.70 \mathrm{~g})$ and catalyst 6, and purified by flash chromatography (hexanes/ ethyl acetate 50:1). Yield: $0.62 \mathrm{~g}, 94 \%$. White solid. Mp $80-81{ }^{\circ} \mathrm{C}$ (from hexane) $[\alpha]_{\mathrm{D}}{ }^{25}=-100.9\left(\mathrm{c}=1.0, \mathrm{CH}_{2} \mathrm{Cl}_{2}\right)$. ${ }^{1} \mathrm{H}$ NMR $\left(300 \mathrm{MHz}, \mathrm{CDCl}_{3}\right): \delta 0.08(\mathrm{~s}, 3 \mathrm{H}) ; 0.16(\mathrm{~s}, 3 \mathrm{H})$; $0.85-1.01(\mathrm{~m}, 2 \mathrm{H}) ; 0.89(\mathrm{~s}, 9 \mathrm{H}) ; 0.91(\mathrm{~d}, J=6.6 \mathrm{~Hz}, 3 \mathrm{H})$; $1.02(\mathrm{~m}, 1 \mathrm{H}) ; 1.16(\mathrm{~s}, 3 \mathrm{H}) ; 1.20(\mathrm{~s}, 3 \mathrm{H}) ; 1.41-1.53(\mathrm{~m}$, $2 \mathrm{H}) ; 1.59(\mathrm{~m}, 1 \mathrm{H}) ; 1.70(\mathrm{~m}, 1 \mathrm{H}) ; 1.92(\mathrm{~m}, 1 \mathrm{H}) ; 2.14(\mathrm{~m}$, $1 \mathrm{H}) ; 2.30(\mathrm{~m}, 1 \mathrm{H}) ; 2.93$ (ddd, $J_{1}=13.7 \mathrm{~Hz}, J_{2}=8.3 \mathrm{~Hz}, J_{3}$ $=1.9 \mathrm{~Hz}, 1 \mathrm{H}) ; 3.20\left(\mathrm{ddd}, J_{1}=13.7 \mathrm{~Hz}, J_{2}=9.1 \mathrm{~Hz}, J_{3}=1.6\right.$ $\mathrm{Hz}, 1 \mathrm{H}) ; 3.46\left(\mathrm{td}, J_{l}=10.6 \mathrm{~Hz}, J_{2}=3.8 \mathrm{~Hz}, 1 \mathrm{H}\right) ; 4.54(\mathrm{~s}$, $1 \mathrm{H}) ; 5.05\left(\mathrm{dd}, J_{1}=10.5 \mathrm{~Hz}, J_{2}=1.9 \mathrm{~Hz}, 1 \mathrm{H}\right) ; 5.42(\mathrm{~d}, J=$ $11.2 \mathrm{~Hz}, 1 \mathrm{H}) ; 5.43\left(\mathrm{dd}, J_{1}=17.1 \mathrm{HZ}, J_{2}=1.9 \mathrm{~Hz}\right) ; 5.72(\mathrm{dt}$, $\left.J_{1}=11.2 \mathrm{~Hz}, J_{2}=5.6 \mathrm{~Hz}, 1 \mathrm{H}\right) ; 6.12\left(\mathrm{dd}, J_{1}=17.0 \mathrm{~Hz}, J_{2}=\right.$ $10.5 \mathrm{~Hz}, 1 \mathrm{H}) .{ }^{13} \mathrm{C}$ NMR $\left(75 \mathrm{MHz}, \mathrm{CDCl}_{3}\right): \delta-2.9\left(\mathrm{CH}_{3}\right) ;-$ $2.5\left(\mathrm{CH}_{3}\right) ; 18.7(\mathrm{C}) ; 22.3\left(\mathrm{CH}_{3}\right) ; 22.8\left(\mathrm{CH}_{3}\right) ; 25.4\left(\mathrm{CH}_{2}\right)$; $26.0\left(3 \mathrm{CH}_{3}\right) ; 28.5\left(\mathrm{CH}_{3}\right) ; 30.0\left(\mathrm{CH}_{2}\right) ; 31.4(\mathrm{CH}) ; 35.2$ $\left(\mathrm{CH}_{2}\right) ; 41.3\left(\mathrm{CH}_{2}\right) ; 41.7\left(\mathrm{CH}_{2}\right) ; 46.8(\mathrm{CH}) ; 57.8(\mathrm{C}) ; 77.1$ $(\mathrm{CH}) ; 80.3(\mathrm{C}) ; 91.3(\mathrm{CH}) ; 112.6\left(\mathrm{CH}_{2}\right) ; 128.9(\mathrm{CH}) ; 134.9$ $(\mathrm{CH}) ; 141.2(\mathrm{CH})$. IR (nujol dispersion): 3080, 1640, 740, $670 \mathrm{~cm}^{-1}$. HRMS calcd for $\mathrm{C}_{24} \mathrm{H}_{44} \mathrm{NO}_{2} \mathrm{Si}[\mathrm{M}+\mathrm{H}]^{+}$ 406.3136, found 406.3142

\section{(3R,4a $R, 5 \mathrm{a} S, 6 R, 13 \mathrm{a} S, Z)-3,8,13,13-t e t r a m e t h y l-6-(2-$ methylallyl)-2,3,4,4a,5a,6,10,11,13,13a-decahydro- $1 H, 7 H$-benzo[5,6] $[1,3]$ oxazino $[3,2-a]$ azocin-6-ol}

This compound was obtained from 19 e (1.0 g) and ca 6, and purified by flash chromatography (hexanes/ ethyl acetate 50:1). Yield: $0.46 \mathrm{~g}, 50 \%$. Colorless oil $[\alpha]_{\mathrm{D}}^{25}=-5.6$ $\left(\mathrm{c}=1.0, \mathrm{CH}_{2} \mathrm{Cl}_{2}\right) .{ }^{1} \mathrm{H}$ NMR $\left(300 \mathrm{MHz}, \mathrm{CDCl}_{3}\right): \delta 0.85-$ $0.98(\mathrm{~m}, 2 \mathrm{H}) ; 0.91(\mathrm{~d}, J=5.9 \mathrm{~Hz}, 3 \mathrm{H}) ; 1.06(\mathrm{~m}, 1 \mathrm{H}) ; 1.13$ $(\mathrm{s}, 3 \mathrm{H}) ; 1.17(\mathrm{~s}, 3 \mathrm{H}) ; 1.22-1.33(\mathrm{~m}, 2 \mathrm{H}) ; 1.44(\mathrm{~m}, 1 \mathrm{H})$; $1.57(\mathrm{~m}, 1 \mathrm{H}) ; 1,67(\mathrm{~m}, 1 \mathrm{H}) ; 1.77(\mathrm{~s}, 3 \mathrm{H}) ; 1.87(\mathrm{~s}, 3 \mathrm{H}) ; 2.05$ $(\mathrm{m}, 1 \mathrm{H}) ; 2.18(\mathrm{~d}, J=13.5 \mathrm{~Hz}, 1 \mathrm{H}) ; 2,24(\mathrm{~m}, 1 \mathrm{H}) ; 2.42(\mathrm{~d}, J$ $=14.5 \mathrm{~Hz}, 1 \mathrm{H}) ; 2.47(\mathrm{~d}, J=14.5 \mathrm{~Hz}, 1 \mathrm{H}) ; 2.61(\mathrm{~d}, J=13.5$ $\mathrm{Hz}, 1 \mathrm{H}) ; 2.82(\mathrm{~m}, 1 \mathrm{H}) ; 3.21\left(\mathrm{ddd}, J_{1}=16.1 \mathrm{~Hz}, J_{2}=9.4 \mathrm{~Hz}\right.$, $\left.J_{3}=2.1 \mathrm{~Hz}, 1 \mathrm{H}\right) ; 3.45\left(\mathrm{td}, J_{l}=10.6 \mathrm{~Hz}, J_{2}=3.9 \mathrm{~Hz}, 1 \mathrm{H}\right) ;$ $4.41(\mathrm{~s}, 1 \mathrm{H}) ; 4.77(\mathrm{~m}, 1 \mathrm{H}) ; 4.85(\mathrm{~m}, 1 \mathrm{H}) ; 5.54(\mathrm{~m}, 1 \mathrm{H})$. ${ }^{13} \mathrm{C}$ NMR (75 MHz, CDCl 3$): \delta 22.2\left(2 \mathrm{CH}_{3}\right) ; 22.7\left(\mathrm{CH}_{3}\right)$; $25.0\left(\mathrm{CH}_{2}\right) ; 26.7\left(\mathrm{CH}_{3}\right) ; 28.9\left(\mathrm{CH}_{3}\right) ; 31.4(\mathrm{CH}) ; 33.4$ $\left(\mathrm{CH}_{2}\right) ; 35.1\left(\mathrm{CH}_{2}\right) ; 39.1\left(\mathrm{CH}_{2}\right) ; 40.9\left(\mathrm{CH}_{2}\right) ; 41.4\left(\mathrm{CH}_{2}\right)$; $46.0(\mathrm{CH}) ; 49.8\left(\mathrm{CH}_{2}\right) 57.7(\mathrm{C}) ; 75.6(\mathrm{C}) ; 76.9(\mathrm{CH}) ; 88.7$ $(\mathrm{CH}) ; 114.8\left(\mathrm{CH}_{2}\right) ; 126.0(\mathrm{CH}) ; 133.6(\mathrm{CH}) ; 143.5(\mathrm{C})$. IR (film): 3320 (broad), 3090, 1650, $705 \mathrm{~cm}^{-1}$. HRMS calcd for $\mathrm{C}_{22} \mathrm{H}_{38} \mathrm{NO}_{2}[\mathrm{M}+\mathrm{H}]^{+} 348.2897$, found 348.2899.

$(3 R, 4 a R, 5 a S, 6 R, 13 a S, Z)-6-m e t h o x y-3,8,13,13-$ tetramethyl-6-(2-methylallyl)-
2,3,4,4a,5a,6,10,11,13,13a-decahydro-1H,7Hbenzo $[5,6][1,3]$ oxazino $[3,2-a]$ azocine $\quad(24 e)$. This compound was obtained from $20 \mathrm{e}(1.0 \mathrm{~g})$ and catalyst 6 , and purified by flash chromatography (hexanes/ ethyl acetate 25:1). Yield: $0.82 \mathrm{~g}, 88 \%$. Colorless oil $[\alpha]_{\mathrm{D}}{ }^{25}=$ $27.7\left(\mathrm{c}=1.0, \mathrm{CH}_{2} \mathrm{Cl}_{2}\right)$. ${ }^{1} \mathrm{H}$ NMR $\left(300 \mathrm{MHz}, \mathrm{CDCl}_{3}\right): \delta$ $0.80-0.97(\mathrm{~m}, 2 \mathrm{H}) ; 0.90(\mathrm{~d}, J=6.6 \mathrm{~Hz}, 3 \mathrm{H}) ; 1.01(\mathrm{~m}, 1 \mathrm{H})$; $1.17(\mathrm{~s}, 6 \mathrm{H}) ; 1.28(\mathrm{~m}, 1 \mathrm{H}) ; 1.42(\mathrm{~m}, 1 \mathrm{H}) ; 1.56(\mathrm{~m}, 1 \mathrm{H})$ $1.67(\mathrm{~m}, 1 \mathrm{H}) ; 1.78(\mathrm{~d}, J=1.5 \mathrm{~Hz}, 3 \mathrm{H}) ; 1.80(\mathrm{~m}, 1 \mathrm{H}) ; 1.84$ $(\mathrm{s}, 3 \mathrm{H}) ; 2.03(\mathrm{~d}, J=16.1 \mathrm{~Hz}, 1 \mathrm{H}) ; 2.15-2.21(\mathrm{~m}, 2 \mathrm{H}) ; 2.36$ $(\mathrm{d}, J=15.1 \mathrm{~Hz}, 1 \mathrm{H}) ; 2.45(\mathrm{~d}, J=15.1 \mathrm{~Hz}, 1 \mathrm{H}) ; 2.87(\mathrm{~m}$, $1 \mathrm{H}) ; 3.00\left(\mathrm{dt}, J_{1}=16.3 \mathrm{~Hz}, J_{2}=5.3 \mathrm{~Hz}, 1 \mathrm{H}\right) ; 3.21\left(\mathrm{dt}, J_{1}=\right.$ $\left.16.3 \mathrm{~Hz}, J_{2}=6.2 \mathrm{~Hz}, 1 \mathrm{H}\right) ; 3.27(\mathrm{~s}, 3 \mathrm{H}) ; 3.37\left(\mathrm{td}, J_{l}=10.6\right.$ $\left.\mathrm{Hz}, J_{2}=3.9 \mathrm{~Hz}, 1 \mathrm{H}\right) ; 4.61(\mathrm{~s}, 1 \mathrm{H}) ; 4.83(\mathrm{~m}, 1 \mathrm{H}) ; 4.89(\mathrm{~m}$, $1 \mathrm{H}) ; 5.40(\mathrm{~m}, 1 \mathrm{H}) \cdot{ }^{13} \mathrm{C} \mathrm{NMR}\left(75 \mathrm{MHz} \mathrm{CDCl}_{3}\right): \delta 20.8$ $\left(\mathrm{CH}_{3}\right) ; 22.3\left(\mathrm{CH}_{3}\right) ; 24.0\left(\mathrm{CH}_{3}\right) ; 25.1\left(\mathrm{CH}_{2}\right) ; 27.1\left(\mathrm{CH}_{3}\right) ;$ $29.6\left(\mathrm{CH}_{3}\right) ; 31.4(\mathrm{CH}) ; 32.8\left(\mathrm{CH}_{2}\right) ; 35.1\left(\mathrm{CH}_{2}\right) ; 37.5$ $\left(\mathrm{CH}_{2}\right) ; 39.3\left(\mathrm{CH}_{2}\right) ; 41.4\left(\mathrm{CH}_{2}\right) ; 42.1\left(\mathrm{CH}_{2}\right) ; 47.1(\mathrm{CH}) ; 50.5$ $\left(\mathrm{CH}_{3}\right) ; 58.1(\mathrm{C}) ; 76.1(\mathrm{CH}) ; 81.1(\mathrm{C}) ; 86.9(\mathrm{CH}) ; 114.0$ $\left(\mathrm{CH}_{2}\right) ; 124.8(\mathrm{CH}) ; 130.5(\mathrm{C}) ; 143.2$ (C) IR (film): 3075 $1640,735,630 \mathrm{~cm}^{-1}$. HRMS calcd for $\mathrm{C}_{23} \mathrm{H}_{40} \mathrm{NO}_{2}[\mathrm{M}+\mathrm{H}]^{2}$ 362.3054 , found 362.3063 .

\section{$(3 R, 4 \mathrm{a} R, 5 \mathrm{a} S, 6 S, 13 \mathrm{a} S, Z)-6-$ methoxy-3,8,13,13-} tetramethyl-6-(2-methylallyl)-

$2,3,4,4 a, 5 a, 6,10,11,13,13 a-d e c a h y d r o-1 H, 7 H-$ benzo $[5,6][1,3]$ oxazino $[3,2-a]$ azocine $\quad(25 \mathrm{e})$. This compound was obtained from $20 \mathrm{e}(1.0 \mathrm{~g})$ and catalyst $\mathbf{6}$ and purified by flash chromatography (hexanes/ ethyl acetate $25: 1)$. Yield: $0.02 \mathrm{~g}, 2 \%$. Colorless oil $[\alpha]_{\mathrm{D}}^{25}=-28.4$ $\left(\mathrm{c}=0.3, \mathrm{CH}_{2} \mathrm{Cl}_{2}\right) .{ }^{\mathrm{l}} \mathrm{H} \mathrm{NMR}\left(300 \mathrm{MHz}, \mathrm{CDCl}_{3}\right): \delta(\mathrm{m}, 1 \mathrm{H})$ $0.82-0.97(\mathrm{~m}, 2 \mathrm{H}) ; 0.90(\mathrm{~d}, J=6.5 \mathrm{~Hz}, 3 \mathrm{H}) ; 1.08(\mathrm{~m}, 1 \mathrm{H})$; $1.12(\mathrm{~s}, 3 \mathrm{H}) ; 1.14(\mathrm{~s}, 3 \mathrm{H}) ; 1.27(\mathrm{~m}, 1 \mathrm{H}) ; 1.46(\mathrm{~m}, 1 \mathrm{H}) ; 1.54$ $(\mathrm{m}, 1 \mathrm{H}) ; 1.66(\mathrm{~m}, 1 \mathrm{H}) ; 1.77(\mathrm{~d}, J=1.5 \mathrm{~Hz}, 3 \mathrm{H}) ; 1.82(\mathrm{~s}$ $3 \mathrm{H}) ; 1.88(\mathrm{~m}, 1 \mathrm{H}) ; 2.05-2.15(\mathrm{~m}, 2 \mathrm{H}) ; 2.30(\mathrm{~d}, J=15.0 \mathrm{~Hz}$, $1 \mathrm{H}) ; 2.37(\mathrm{~d}, 1 \mathrm{H}, \mathrm{CHH}-\mathrm{C}=, J=15.4 \mathrm{~Hz}) ; 2.52(\mathrm{~d}, 1 \mathrm{H}$ C $\underline{H} \mathrm{H}-\mathrm{C}=, J=15.0 \mathrm{~Hz}) ; 2.58(\mathrm{~d}, J=15.4 \mathrm{~Hz}, 1 \mathrm{H}) ; 2.85(\mathrm{dt}$, $\left.J_{1}=15.9 \mathrm{~Hz}, J_{2}=4.1 \mathrm{~Hz}, 1 \mathrm{H}\right) ; 3.31\left(\mathrm{td}, J_{1}=10.5 \mathrm{~Hz}, J_{2}=\right.$ $3.9 \mathrm{~Hz}, 1 \mathrm{H}) ; 3.35(\mathrm{~s}, 3 \mathrm{H}) ; 3.75\left(\mathrm{ddd}, J_{1}=15.9 \mathrm{~Hz}, J_{2}=\right.$ $\left.11.0 \mathrm{~Hz}, J_{3}=10.5 \mathrm{~Hz}, 1 \mathrm{H}\right) ; 4.47(\mathrm{~s}, 1 \mathrm{H}) ; 4.86(\mathrm{~s}, 2 \mathrm{H}) ; 5.44$ $(\mathrm{m}, 1 \mathrm{H}){ }^{\mathrm{P}_{3} \mathrm{C}} \mathrm{NMR}\left(75 \mathrm{MHz}, \mathrm{CDCl}_{3}\right): 20.6\left(\mathrm{CH}_{3}\right) ; 21.2$ $\left(\mathrm{CH}_{3}\right) ; 24.4\left(\mathrm{CH}_{3}\right) ; 25.1\left(\mathrm{CH}_{2}\right) ; 26.4\left(\mathrm{CH}_{3}\right) ; 29.6\left(\mathrm{CH}_{3}\right)$ $31.4(\mathrm{CH}) ; 32.9\left(\mathrm{CH}_{2}\right) ; 35.1\left(\mathrm{CH}_{2}\right) ; 38.9\left(\mathrm{CH}_{2}\right) ; 39.8$ $\left(\mathrm{CH}_{2}\right) ; 41.5\left(\mathrm{CH}_{2}\right) ; 42.1\left(\mathrm{CH}_{2}\right) ; 46.9(\mathrm{CH}) ; 51.0\left(\mathrm{CH}_{3}\right) ; 57.4$ (C); $76.7(\mathrm{CH}) ; 81.1(\mathrm{C}) ; 88.5(\mathrm{CH}) ; 113.9\left(\mathrm{CH}_{2}\right) ; 125.9$ (CH); 131.3 (C=; 143.3 (C). IR (film): $3069,1636,620 \mathrm{~cm}$ HRMS calcd for $\mathrm{C}_{23} \mathrm{H}_{40} \mathrm{NO}_{2}[\mathrm{M}+\mathrm{H}]^{+} 362.3054$, found 362.3057

General procedure for hydrogenation of compounds $7 \mathbf{a}$, 7f and 14.

A mixture of the unsaturated oxazine $(0.15 \mathrm{~g})$ and $10 \mathrm{wt} \%$ palladium on carbon $(15 \mathrm{mg})$ in ethanol $(1 \mathrm{~mL})$ was stirred at room temperature under $\mathrm{H}_{2}$ at atmospheric pressure until the reaction was complete (TLC, 16-24 h). The catalyst was separated by filtration over a pad of Celite, after which the solvent was eliminated on a rotavapor, and the residue was purified by flash chromatography on silica gel.

\section{$(2 S, 4 a S, 7 R, 8 a R)-2-((2 R, 4 R)-2$-isobutyl-4-} methyltetrahydro-2H-pyran-2-yl)-4,4,7-

trimethyloctahydro- $2 H$-benzo $[\boldsymbol{e}][1,3]$ oxazine (9). This compound was obtained from $7 \mathbf{f}(0.15 \mathrm{~g})$ and, and purified by flash chromatography (hexanes/ ethyl acetate 12:1). Yield: $55 \mathrm{mg}_{35} 46 \%$. White solid. $\mathrm{Mp} 65-67{ }^{\circ} \mathrm{C}$ (from pentane $) .[\alpha]_{\mathrm{D}}{ }^{25}=-15.5\left(\mathrm{c}=0.5, \mathrm{CH}_{2} \mathrm{Cl}_{2}\right) .{ }^{1} \mathrm{H}$ NMR $(300$ $\left.\mathrm{MHz} \mathrm{CDCl}_{3}\right): \delta 0.80-0.96(\mathrm{~m}, 2 \mathrm{H}) ; 0.84(\mathrm{~d}, J=6.5 \mathrm{~Hz}$ $3 \mathrm{H}) ; 0.89(\mathrm{~d}, J=6.5 \mathrm{~Hz}, 3 \mathrm{H},) ; 0.90(\mathrm{~d}, J=6.6 \mathrm{~Hz}, 3 \mathrm{H})$; $0.92(\mathrm{~d}, J=6.6 \mathrm{~Hz}, 3 \mathrm{H}) ; 0.98-1.13(\mathrm{~m}, 2 \mathrm{H}) ; 1.00(\mathrm{~s}, 3 \mathrm{H})$; $1.04(\mathrm{~s}, 3 \mathrm{H}) ; 1.25(\mathrm{~m}, 1 \mathrm{H}) ; 1.29(\mathrm{~m}, 1 \mathrm{H}), 1.36-1.52(\mathrm{~m}$ $4 \mathrm{H}) ; 1.59-1.69(\mathrm{~m}, 2 \mathrm{H}) ; 1.71-1.80(\mathrm{~m}, 2 \mathrm{H}) ; 1.94(\mathrm{~m}, 1 \mathrm{H})$ $2.03(\mathrm{~m}, 1 \mathrm{H}) ; 3.27\left(\mathrm{td}, J_{1}=10.4 \mathrm{~Hz}, J_{2}=4.0 \mathrm{~Hz}, 1 \mathrm{H}\right) ; 3.66$ $(\mathrm{m}, 1 \mathrm{H}) ; 3.91(\mathrm{~m}, 1 \mathrm{H}) ; 4.39(\mathrm{~s}, 1 \mathrm{H}) .{ }^{13} \mathrm{C} \mathrm{NMR}(75 \mathrm{MHz}$ $\left.\mathrm{CDCl}_{3}\right): \delta 19.7\left(\mathrm{CH}_{3}\right) ; 22.3\left(\mathrm{CH}_{3}\right) ; 23.3\left(\mathrm{CH}_{3}\right) ; 23.6(\mathrm{CH})$ $24.8\left(\mathrm{CH}_{3}\right) ; 25.3\left(\mathrm{CH}_{3}\right) ; 25.6(\mathrm{CH}) ; 25.7\left(\mathrm{CH}_{2}\right) ; 30.1\left(\mathrm{CH}_{3}\right)$ $31.4(\mathrm{CH}) ; 24.2\left(\mathrm{CH}_{2}\right) ; 35.1\left(\mathrm{CH}_{2}\right) ; 39.6\left(\mathrm{CH}_{2}\right) ; 42.0$ $\left(\mathrm{CH}_{2}\right) ; 46.2\left(\mathrm{CH}_{2}\right) ; 50.8(\mathrm{C}) ; 52.2(\mathrm{CH}) ; 62.8\left(\mathrm{CH}_{2}\right) ; 75.3$ 
$(\mathrm{CH}) ; 76.6(\mathrm{C}) ; 85.1(\mathrm{CH})$. HRMS calcd for $\mathrm{C}_{21} \mathrm{H}_{40} \mathrm{NO}_{2}$ $[\mathrm{M}+\mathrm{H}]^{+} 338.3054$, found 338.3056

$(2 S, 4 a S, 7 R, 8 a R)-2-((R)-2$-ethyltetrahydrofuran-2-yl)4,4,7-trimethyloctahydro- $2 H$-benzo $[e][1,3]$ oxazine $(10)$. This compound was obtained from $7 \mathbf{a}(0.15 \mathrm{~g})$ and purified by flash chromatography (hexanes/ ethyl acetate 12:1). Yield: $61 \mathrm{mg}, 53 \%$. Colorless oil. $[\alpha]_{\mathrm{D}}^{25}=-8.4(\mathrm{c}=0.5$, $\mathrm{CH}_{2} \mathrm{Cl}_{2}$ ). ${ }^{1} \mathrm{H} \mathrm{NMR}\left(300 \mathrm{MHz}, \mathrm{CDCl}_{3}\right): \delta$ 0.76-1.24 (m, $4 \mathrm{H}) ; 0.83(\mathrm{t}, J=7.6 \mathrm{~Hz}, 3 \mathrm{H}) ; 0.88(\mathrm{~d}, J=6.5 \mathrm{~Hz}, 3 \mathrm{H}) ; 1.00$ $(\mathrm{s}, 3 \mathrm{H}) ; 1.03(\mathrm{~s}, 3 \mathrm{H}) ; 1.42(\mathrm{~m}, 1 \mathrm{H}) ; 1.50-1.68(\mathrm{~m}, 5 \mathrm{H})$; $1.76(\mathrm{~m}, 1 \mathrm{H}) ; 1.85(\mathrm{~m}, 1 \mathrm{H}) ; 1.93(\mathrm{~m}, 1 \mathrm{H}) ; 2.08(\mathrm{~m}, 1 \mathrm{H})$; $3.29\left(\mathrm{td}, J_{1}=10.4 \mathrm{~Hz}, J_{2}=4.0 \mathrm{~Hz}, 1 \mathrm{H}\right) ; 3.74-3.90(\mathrm{~m}, 2 \mathrm{H})$; $4.13(\mathrm{~s}, 1 \mathrm{H}) \cdot{ }^{13} \mathrm{C}$ NMR $\left(75 \mathrm{MHz}, \mathrm{CDCl}_{3}\right): \delta 8.5\left(\mathrm{CH}_{3}\right)$; $19.7\left(\mathrm{CH}_{3}\right) ; 22.3\left(\mathrm{CH}_{3}\right) ; 25.8\left(\mathrm{CH}_{2}\right) ; 26.6\left(\mathrm{CH}_{2}\right) ; 29.5$ $\left(\mathrm{CH}_{2}\right) ; 29.9\left(\mathrm{CH}_{3}\right) ; 30.8\left(\mathrm{CH}_{2}\right) ; 31.4(\mathrm{CH}) ; 35.1\left(\mathrm{CH}_{2}\right) ; 41.8$ $\left(\mathrm{CH}_{2}\right) ; 50.7(\mathrm{C}) ; 51.6(\mathrm{CH}) ; 69.2\left(\mathrm{CH}_{2}\right) ; 75.1(\mathrm{CH})$; 85.6 (CH); $86.3(\mathrm{C})$. HRMS calcd for $\mathrm{C}_{17} \mathrm{H}_{32} \mathrm{NO}_{2}[\mathrm{M}+\mathrm{H}]^{+}$ 282.2428 , found 282.2432 .

General method for the elimination of the chiral auxiliary in 7a and 7c-g. A mixture of the oxazine 7 (2 $\mathrm{mmol})$, ethanol $(20 \mathrm{~mL})$ and a $3 \%$ aqueous solution of $\mathrm{HCl}$ $(20 \mathrm{~mL})$ was heated at reflux until the reaction was finished (TLC and ${ }^{1} \mathrm{H}-\mathrm{NMR}$ were used to monitor the reaction for disappearance of starting material). The mixture was cooled to $0{ }^{\circ} \mathrm{C}$, the $\mathrm{pH}$ was ajusted to $7-8$ by addition of a $15 \%$ solution of $\mathrm{NaOH}$ and $\mathrm{NaBH}_{4}(226 \mathrm{mg}$, $6 \mathrm{mmol}$ ) was added. The mixture was further stirred for $3 \mathrm{~h}$ at $0{ }^{\circ} \mathrm{C}$. Then $40 \mathrm{~mL}$ of $\mathrm{H}_{2} \mathrm{O}$ was added and the mixture was stirred for $1 \mathrm{~h}$ at room temperature. The aqueous mixture was extracted with ethyl ether $(4 \times 20 \mathrm{~mL})$. The combined organic layers were washed with brine, dried over $\mathrm{MgSO}_{4}$, concentrated by distillation at atmospheric pressure and the resulting residue was purified by flash chromatography on silica gel.

(R)-(2-vinyl-2,5-dihydrofuran-2-yl)methanol (15a). This compound was obtained from $7 \mathbf{a}(0.9 \mathrm{~g})$ and purified by flash chromatography (hexanes/ ethyl ether 15:1). Yield: $0.22 \mathrm{~g}, 72 \%$. Colorless oil. $[\alpha]^{25}=+325.0 \quad(\mathrm{c}=0.5$, $\mathrm{CH}_{2} \mathrm{Cl}_{2}$ ). ${ }^{1} \mathrm{H}$ NMR $\left(300 \mathrm{MHz}, \mathrm{CDCl}_{3}\right): \delta 1.88$ (s broad, $1 \mathrm{H}) ; 3.62(\mathrm{~m}, 2 \mathrm{H}) ; 4.71-4.78(\mathrm{~m}, 2 \mathrm{H}) ; 5.18\left(\mathrm{dd}, J_{1}=10.7\right.$ $\left.\mathrm{Hz}, J_{2}=1.5 \mathrm{~Hz}, 1 \mathrm{H}\right) ; 5.33\left(\mathrm{dd}, J_{1}=17.3 \mathrm{~Hz}, J_{2}=1.5 \mathrm{~Hz}\right.$, $1 \mathrm{H}) ; 5.76(\mathrm{~m}, 1 \mathrm{H}) ; 5.90\left(\mathrm{dd}, J_{1}=17.1 \mathrm{~Hz}, J_{2}=10.7 \mathrm{~Hz}\right.$, $1 \mathrm{H}) ; 6.02(\mathrm{~m}, 1 \mathrm{H}) .{ }^{13} \mathrm{C} \mathrm{NMR}\left(75 \mathrm{MHz}, \mathrm{CDCl}_{3}\right): \delta 67.1$ $\left(\mathrm{CH}_{2}\right) ; 75.4\left(\mathrm{CH}_{2}\right) ; 93.1(\mathrm{C}) ; 115.1\left(\mathrm{CH}_{2}\right) ; 128.2(\mathrm{CH})$; $128.8(\mathrm{CH}) ; 137.6$ ( CH). IR (film): 3245 (broad), 1640, $1420,815,725 \mathrm{~cm}^{-1}$. HRMS calcd for $\mathrm{C}_{7} \mathrm{H}_{10} \mathrm{NaO}_{2}[\mathrm{M}+$ $\mathrm{Na}^{+} 149.0573$, found 149.0578 .

(R)-(3-methyl-2-(prop-1-en-2-yl)-2,5-dihydrofuran-2yl)metanol $(\mathbf{1 5 c})$. This compound was obtained from $7 \mathrm{c}$ $(1.0 \mathrm{~g})$ and purified by flash chromatography (hexanes/ ethyl ether 15:1). Yield: $0.27 \mathrm{~g}, 70 \%$. Colorless oil. $[\alpha]_{\mathrm{D}}{ }^{25}=$ $-136.5\left(\mathrm{c}=0.6, \mathrm{CH}_{2} \mathrm{Cl}_{2}\right) .{ }^{1} \mathrm{H}$ NMR $\left(300 \mathrm{MHz}, \mathrm{CDCl}_{3}\right): \delta$ $1.66(\mathrm{~m}, 3 \mathrm{H}) ; 1.71(\mathrm{~m}, 3 \mathrm{H}) ; 2.08(\mathrm{~s}$ broad, $1 \mathrm{H}) ; 3.80(\mathrm{~m}$, $2 \mathrm{H}) ; 4.64(\mathrm{~m}, 2 \mathrm{H}) ; 4.80(\mathrm{~s}, 1 \mathrm{H}) ; 4.93(\mathrm{~s}, 1 \mathrm{H}) ; 5.68(\mathrm{~m}, 1 \mathrm{H})$. ${ }^{13} \mathrm{C}$ NMR $\left(75 \mathrm{MHz}, \mathrm{CDCl}_{3}\right): \delta 11.8\left(\mathrm{CH}_{3}\right) ; 18.9\left(\mathrm{CH}_{3}\right)$; $64.2\left(\mathrm{CH}_{2}\right) ; 75.0\left(\mathrm{CH}_{2}\right) ; 95.2(\mathrm{C}) ; 111.1\left(\mathrm{CH}_{2}\right) ; 122.7$ (CH); 136.6 (C); 145.3 (C). IR (film): 3450 (broad), 3090 , $1640,900,860,780,765,680 \mathrm{~cm}^{-1}$. HRMS calcd for $\mathrm{C}_{9} \mathrm{H}_{14} \mathrm{NaO}_{2}[\mathrm{M}+\mathrm{Na}]^{+}$177.0886, found 177.0887.

(R)-(3,4-dimethyl-2-(prop-1-en-2-yl)-2,5-dihydrofuran2-yl)metanol (15d). This compound was obtained from 7d $(0.8 \mathrm{~g})$ and purified by flash chromatography (hexanes/ ethyl ether 15:1). Yield: $0.29 \mathrm{~g}, 88 \%$. Colorless oil. $[\alpha]_{\mathrm{D}}{ }^{25}=$ $-112.7\left(\mathrm{c}=1.5, \mathrm{CH}_{2} \mathrm{Cl}_{2}\right) .{ }^{1} \mathrm{H}$ NMR $\left(300 \mathrm{MHz} \mathrm{CDCl}_{3}\right): \delta$ $1.53(\mathrm{~s}, 3 \mathrm{H}) ; 1.67(\mathrm{~s}, 3 \mathrm{H}) ; 1.69(\mathrm{~s}, 3 \mathrm{H}) ; 2.02(\mathrm{~s}$ broad, $1 \mathrm{H})$; $3.78(\mathrm{~m}, 2 \mathrm{H}) ; 4.56(\mathrm{~m}, 2 \mathrm{H}) ; 4.77(\mathrm{~s}, 1 \mathrm{H}) ; 4.90(\mathrm{~s}, 1 \mathrm{H}) .{ }^{3} \mathrm{C}$ NMR $\left(75 \mathrm{MHz}, \mathrm{CDCl}_{3}\right): \delta 9.2\left(\mathrm{CH}_{3}\right) ; 10.2\left(\mathrm{CH}_{3}\right) ; 19.0$ $\left(\mathrm{CH}_{3}\right) ; 64.3\left(\mathrm{CH}_{2}\right) ; 78.8\left(\mathrm{CH}_{2}\right) ; 96.6(\mathrm{C}) ; 110.7\left(\mathrm{CH}_{2}\right)$; 127.8 (C); 130.0 (C); 145.3 (C). IR (film): 3440 (broad), $3085,1640,900,850,680 \mathrm{~cm}^{-1}$. HRMS calcd for $\mathrm{C}_{10} \mathrm{H}_{16} \mathrm{NaO}_{2}[\mathrm{M}+\mathrm{Na}]^{+} 191.1043$, found 191.1049.

(R)-(2-allyl-3,6-dihydro-2H-pyran-2-yl)metanol (15e). This compound was obtained from 7 e $(0.75 \mathrm{~g})$ and purified by flash chromatography (hexanes/ ethyl ether 15:1). Yield: $0.20 \mathrm{~g}, 68 \%$. Colorless oil. $[\alpha]_{\mathrm{D}}{ }^{25}=+42.2(\mathrm{c}=1.0$, $\left.\mathrm{CH}_{2} \mathrm{Cl}_{2}\right) .{ }^{1} \mathrm{H}$ NMR $\left(300 \mathrm{MHz}, \mathrm{CDCl}_{3}\right): \delta 1.61(\mathrm{~m}, 1 \mathrm{H})$ $2.14(\mathrm{~m}, 1 \mathrm{H}) ; 2.28-2.49(\mathrm{~m}, 3 \mathrm{H}) ; 3.52(\mathrm{~s}, 2 \mathrm{H}) ; 4.12-4.25$ $(\mathrm{m}, 2 \mathrm{H}) ; 5.08-5.31(\mathrm{~m}, 2 \mathrm{H}) ; 5.89-5.70(\mathrm{~m}, 3 \mathrm{H}) .{ }^{113} \mathrm{C}$ NMR $\left(75 \mathrm{MHz}, \mathrm{CDCl}_{3}\right): \delta 28.4\left(\mathrm{CH}_{2}\right) ; 37.8\left(\mathrm{CH}_{2}\right) ; 60.8\left(\mathrm{CH}_{2}\right)$ $66.0\left(\mathrm{CH}_{2}\right) ; 73.7(\mathrm{C}) ; 118.2\left(\mathrm{CH}_{2}\right) ; 122.5(\mathrm{CH}) ; 124.8$ $(\mathrm{CH}) ; 133.3$ (CH). IR (film): 3435 (broad), 1640, 1100 , $740,655 \mathrm{~cm}^{-1}$. HRMS calcd for $\mathrm{C}_{9} \mathrm{H}_{14} \mathrm{NaO}_{2}[\mathrm{M}+\mathrm{Na}]^{+}$ 177.0886, found 177.0884 .

(R)-(4-methyl-2-(2-methylallyl)-3,6-dihydro-2H-pyran2-yl)metanol (15f). This compound was obtained from $\mathbf{7 f}$ $(0.8 \mathrm{~g})$ and purified by flash chromatography (hexanes/ ethyl ether 15:1). Yield: $0.25 \mathrm{~g}, 73 \%$. Colorless oil. $[\alpha]_{\mathrm{D}}{ }^{25}=$ $+37.7\left(\mathrm{c}=0.6, \mathrm{CH}_{2} \mathrm{Cl}_{2}\right)$. $\mathrm{H}$ NMR $\left(300 \mathrm{MHz}, \mathrm{CDCl}_{3}\right): \delta$ $1.71(\mathrm{~d}, J=1.2 \mathrm{~Hz}, 3 \mathrm{H}) ; 1.82(\mathrm{~s}, 3 \mathrm{H}) ; 1.88-2.07(\mathrm{~m}, 3 \mathrm{H})$; $2.29(\mathrm{~d}, J=13.6 \mathrm{~Hz}, 1 \mathrm{H})-2.36(\mathrm{~d}, J=13.6 \mathrm{~Hz}, 1 \mathrm{H}) ; 3.55$ $(\mathrm{m}, 2 \mathrm{H}) ; 4.14-4.23(\mathrm{~m}, 2 \mathrm{H}) ; 4.76(\mathrm{~m}, 1 \mathrm{H}) ; 4.89(\mathrm{~m}, 1 \mathrm{H})$ $5.41(\mathrm{~m}, 1 \mathrm{H}) .{ }^{13} \mathrm{C} \mathrm{NMR}\left(75 \mathrm{MHz}, \mathrm{CDCl}_{3}\right): \delta 23.3\left(\mathrm{CH}_{3}\right)$ $24.4\left(\mathrm{CH}_{3}\right) ; 33.7\left(\mathrm{CH}_{2}\right) ; 41.5\left(\mathrm{CH}_{2}\right) ; 61.1\left(\mathrm{CH}_{2}\right) ; 66.1$ $\left(\mathrm{CH}_{2}\right) ; 74.5(\mathrm{C}) ; 115.0\left(\mathrm{CH}_{2}\right) ; 118.1(\mathrm{CH}) ; 130.1(\mathrm{C})$; 142.2 (C). IR (film): 3450 (broad), 3070, 1640, 890, 770 $\mathrm{cm}^{-1}$. HRMS calcd for $\mathrm{C}_{11} \mathrm{H}_{18} \mathrm{NaO}_{2}[\mathrm{M}+\mathrm{Na}]^{+} 205.1199$ found 205.1204

(R)-(4,5-dimethyl-2-(2-methylallyl)-3,6-dihydro-2Hpyran-2-yl)metanol (15g). This compound was obtained from $13(0.65 \mathrm{~g})$ and purified by flash chromatography (hexanes/ ethyl ether 15:1). Yield: $0.18 \mathrm{~g}, 63 \%$. Colorless oil. $[\alpha]_{\mathrm{D}}{ }^{25}=+28.8\left(\mathrm{c}=0.8, \mathrm{CH}_{2} \mathrm{Cl}_{2}\right) .{ }^{1} \mathrm{H}$ NMR $(300 \mathrm{MHz}$ $\left.\mathrm{CDCl}_{3}\right): \delta 1.55(\mathrm{~s}, 3 \mathrm{H}) ; 1.65(\mathrm{~m}, 3 \mathrm{H}) ; 1.82(\mathrm{~m}, 3 \mathrm{H}) ; 1.89$ $2.08(\mathrm{~m}, 3 \mathrm{H}) ; 2.26(\mathrm{~d}, J=13.6 \mathrm{~Hz}, 1 \mathrm{H}) ;-2.35(\mathrm{~d}, J=13.6$ $\mathrm{Hz}, 1 \mathrm{H}) ; 3.47-3.51(\mathrm{~m}, 2 \mathrm{H}) ; 4.97-4.99(\mathrm{~m}, 2 \mathrm{H}) ; 4.76(\mathrm{~m}$ $1 \mathrm{H}) ; 4.89(\mathrm{~m}, 1 \mathrm{H}) .{ }^{3} \mathrm{C} \mathrm{NMR}\left(75 \mathrm{MHz}, \mathrm{CDCl}_{3}\right): \delta 13.7$ $\left(\mathrm{CH}_{3}\right) ; 18.6\left(\mathrm{CH}_{3}\right) ; 24.4\left(\mathrm{CH}_{3}\right) ; 34.6\left(\mathrm{CH}_{2}\right) ; 41.5\left(\mathrm{CH}_{2}\right)$ $64.8\left(\mathrm{CH}_{2}\right) ; 65.9\left(\mathrm{CH}_{2}\right) ; 74.9(\mathrm{C}) ; 114.9\left(\mathrm{CH}_{2}\right) ; 121.9(\mathrm{C})$; 122.6 (C); 142.3 (C). IR (film): 3450 (broad), 3070, 1640 $890 \mathrm{~cm}^{-1}$. HRMS calcd for $\mathrm{C}_{12} \mathrm{H}_{20} \mathrm{NaO}_{2}[\mathrm{M}+\mathrm{Na}]^{+}$ 219.1356 , found 219.1353 .

$(1 R, 2 S, 5 R)-2-(2-((R, Z)-3-m e t h o x y-5-m e t h y l-3-(2-$ methylallyl)-3,4,7,8-tetrahydroazocin-1(2H)-yl)propan2-yl)-5-methylcyclohexan-1-ol (28). To a suspension of $\mathrm{LiAlH}_{4}(0.30 \mathrm{~g}, 7.9 \mathrm{mmol})$ in anhydrous THF $(20 \mathrm{~mL})$ cooled to $-10^{\circ} \mathrm{C}$ and under nitrogen atmosphere was added in portions, dry $\mathrm{AlCl}_{3}(035 \mathrm{~g}, 2.6 \mathrm{mmol})$. The mixture was stirred for $15 \mathrm{~min}$ at $-10^{\circ} \mathrm{C}$ and a solution of $24 \mathrm{e}(0.75 \mathrm{~g}, 2.1$ $\mathrm{mmol})$ in dry THF $(15 \mathrm{~mL})$ was slowly added. The reaction mixture was stirred at room temperature until disappearance of the starting product (TLC) and then it was quenched by addition of $10 \%$ aqueous solution of $\mathrm{NaOH}(1.5 \mathrm{~mL})$. The resulting mixture was filtered, the solid was washed with hot ethyl acetate, and the organic layer was dried over anhydrous $\mathrm{MgSO}_{4}$. The solvent was eliminated under reduced pressure. The residue was purified by flash chromatography (hexanes/ ethyl acetate 2:1). Yield: $0.69 \mathrm{~g}, 92 \%$ White solid. Mp 94-96 ${ }^{\circ} \mathrm{C}$ (from pentane $) .[\alpha]_{\mathrm{D}}{ }^{25}=-35.1\left(\mathrm{c}=1.5, \mathrm{CH}_{2} \mathrm{Cl}_{2}\right) .{ }^{1} \mathrm{H}$ NMR $(300$ $\left.\mathrm{MHz}_{\mathrm{CDCl}}, 333 \mathrm{~K}\right): \delta 0.82-1.06(\mathrm{~m}, 2 \mathrm{H}) ; 0.90(\mathrm{~d}, J=6.5$ $\mathrm{Hz}, 3 \mathrm{H}) ; 0.91(\mathrm{~s}, 3 \mathrm{H}) ; 1.17\left(\mathrm{~s}, 3 \mathrm{H}, \mathrm{CH}_{3}\right) ; 1.38(\mathrm{~m}, 1 \mathrm{H})$; $1.52-1.81(\mathrm{~m}, 3 \mathrm{H}) ; 1.77(\mathrm{~s}, 3 \mathrm{H}) ; 1.81(\mathrm{~s}, 3 \mathrm{H}) ; 1.93(\mathrm{~m}, 1 \mathrm{H})$ $2.02-2.19(\mathrm{~m}, 2 \mathrm{H}) ; 2.2-2.48(\mathrm{~m}, 5 \mathrm{H}) ; 3.20(\mathrm{~m}, 1 \mathrm{H}) ; 3.23(\mathrm{~s}$ $3 \mathrm{H}) ; 3.62\left(\mathrm{td}, J_{1}=10.1 \mathrm{~Hz}, J_{2}=3.9 \mathrm{~Hz}, 1 \mathrm{H}\right) ; 4.88(\mathrm{~m}, 1 \mathrm{H})$ $4.95(\mathrm{~m}, 1 \mathrm{H}) ; 5.52(\mathrm{~m}, 1 \mathrm{H}) ; 7.50(\mathrm{~s}$ broad, $1 \mathrm{H}) .{ }^{13} \mathrm{C}$ NMR $\left(75 \mathrm{MHz}, \mathrm{CDCl}_{3}, 333 \mathrm{~K}\right): \delta 20.2\left(\mathrm{CH}_{3}\right) ; 21.9\left(2 \mathrm{CH}_{3}\right) ; 24.2$ $\left(\mathrm{CH}_{3}\right) ; 25.5\left(\mathrm{CH}_{3}\right) ; 26.6\left(\mathrm{CH}_{2}\right) ; 28.5\left(\mathrm{CH}_{2}\right) ; 31.0(\mathrm{CH})$; $35.3\left(\mathrm{CH}_{2}\right) ; 37.9\left(\mathrm{CH}_{2}\right) ; 40.4\left(\mathrm{CH}_{2}\right) ; 44.7\left(\mathrm{CH}_{2}\right) ; 48.1(\mathrm{CH})$ $48.7\left(\mathrm{CH}_{3}\right) ; 51.8\left(\mathrm{CH}_{2}\right) ; 55.3\left(\mathrm{CH}_{2}\right) ; 63.4(\mathrm{C}) ; 72.3(\mathrm{CH})$ $80.6(\mathrm{C}) ; 113.9\left(\mathrm{CH}_{2}\right) ; 124.5(\mathrm{CH}) ; 137.0(\mathrm{C}) ; 141.8(\mathrm{C})$ IR (nujol dispersion): 3170 (broad); $3075,1640,740 \mathrm{~cm}^{-1}$ HRMS calcd for $\mathrm{C}_{23} \mathrm{H}_{42} \mathrm{NO}_{2}[\mathrm{M}+\mathrm{H}]^{+} 364.3210$, found 364.3218

\section{General method for elimination of the chiral auxiliary} in 25a, 24b, 22c, 24c, 25d and 24e. To a suspension of $\mathrm{LiAlH}_{4}(0.30 \mathrm{~g}, 7.9 \mathrm{mmol})$ in anhydrous THF $(20 \mathrm{~mL})$ cooled to $-10^{\circ} \mathrm{C}$ and under nitrogen atmosphere was added, in portions, dry $\mathrm{AlCl}_{3}(0.35 \mathrm{~g}, 2.6 \mathrm{mmol})$. The mixture was 
stirred for $15 \mathrm{~min}$ at $-10^{\circ} \mathrm{C}$ and a solution of the corresponding benzoxazine $(2.0 \mathrm{mmol})$ in dry THF (15 $\mathrm{mL}$ ) was slowly added. The reaction mixture was stirred at at room temperature until disappearance of the starting product by TLC (for reduction of $\mathbf{2 4 b}$ it was necessary to heat to $60^{\circ} \mathrm{C}$ ), and then it was quenched by addition of $10 \%$ aqueous solution of $\mathrm{NaOH}(1.5 \mathrm{~mL})$. The resulting mixture was filtered, the solid was washed with hot ethyl acetate, and the organic layer was dried over anhydrous $\mathrm{MgSO}_{4}$. The solvent was eliminated under reduced pressure, the residue was redissolved in anhydrous $\mathrm{CH}_{2} \mathrm{Cl}_{2}$ $(30 \mathrm{~mL})$, and PCC $(1.8 \mathrm{~g}, 8.0 \mathrm{mmol})$ and $3 \mathrm{~A}$ molecular sieves $(4.0 \mathrm{~g})$ was added to this solution. The mixture was stirred under nitrogen atmosphere until the oxidation was finished (TLC). The mixture was then treated with a $10 \%$ aqueous solution of $\mathrm{NaOH}(50 \mathrm{~mL})$ and extracted with $\mathrm{CHCl}_{3}(5 \times 25 \mathrm{~mL})$. The organic phase was washed with brine, dried over anhydrous $\mathrm{MgSO}_{4}$ and the solvent were removed under reduced pressure. The residue was redisolved in THF $(30 \mathrm{~mL})$ and $\mathrm{MeOH}(15 \mathrm{ml})$, an aqueous solution of $\mathrm{KOH} 2.5 \mathrm{M}(15 \mathrm{~mL})$ was added and the mixture stirred at room temperature for 5-6 h. After elimination of the THF and $\mathrm{MeOH}$ under reduced pressure, the residue was acidified with a $1 \mathrm{M}$ solution of $\mathrm{HCl}$ to $\mathrm{pH} 2$ and extracted twice with $\mathrm{Et}_{2} \mathrm{O}(2 \times 20 \mathrm{~mL})$. The aqueous solution was neutralized to $\mathrm{pH} 12$ with a $10 \%$ aqueous solution of $\mathrm{NaOH}$ and extracted with $\mathrm{CHCl}_{3}(4 \times 20 \mathrm{~mL})$. The organic layer was washed with brine, dried over $\mathrm{MgSO}_{4}$, and the solvent eliminated under vacuum and the residue was purified by flash chromatography.

\section{(R)-4-methyl-3-(prop-1-en-2-yl)-1,2,3,6-}

tetrahydropyridin-3-ol (29). This compound was obtained from $24 \mathbf{b}(0.65 \mathrm{~g})$ and purified by flash chromatography $\left(\mathrm{CHCl}_{3} / \mathrm{MeOH} 15: 1\right)$. Yield: $0.19 \mathrm{~g}, 60 \%$. Colorless oil. $[\alpha]_{\mathrm{D}}{ }^{25}=-10.5\left(\mathrm{c}=0.8, \mathrm{CH}_{2} \mathrm{Cl}_{2}\right)$. ${ }^{1} \mathrm{H} \mathrm{NMR}$ $\left(300 \mathrm{MHz}, \mathrm{CDCl}_{3}\right): \delta 1.62(\mathrm{~d} J=0.8 \mathrm{~Hz}, 3 \mathrm{H}) ; 1.70(\mathrm{~s}, 3 \mathrm{H}$, $\left.\mathrm{CH}_{3}\right) ; 2.29(\mathrm{~d}, J=12.8 \mathrm{~Hz}, 1 \mathrm{H}) ; 2.96(\mathrm{~d}, J=12.8 \mathrm{~Hz}, 1 \mathrm{H}) ;$ $2.90-3.00(\mathrm{~m}, 2 \mathrm{H}) ; 3.28-3.29(\mathrm{~m}, 2 \mathrm{H}) ; 5.01(\mathrm{~m}, 1 \mathrm{H}) ; 5.15$ $(\mathrm{m}, 1 \mathrm{H}) ; 5.64(\mathrm{~m}, 1 \mathrm{H}) .{ }^{13} \mathrm{C}$ NMR $\left(75 \mathrm{MHz}, \mathrm{CDCl}_{3}\right.$, $333 \mathrm{~K}): \delta 17.3\left(\mathrm{CH}_{3}\right) ; 18.9\left(\mathrm{CH}_{3}\right) ; 45.1\left(\mathrm{CH}_{2}\right) ; 54.1\left(\mathrm{CH}_{2}\right)$; $73.8(\mathrm{C}) ; 112.5\left(\mathrm{CH}_{2}\right) ; 124.9(\mathrm{CH}) ; 135.8(\mathrm{C}) ; 145.8(\mathrm{C})$ IR (film): 3030 (broad), 3025, 1640, $740 \mathrm{~cm}^{-1}$. HRMS calcd for $\mathrm{C}_{9} \mathrm{H}_{16} \mathrm{NO}[\mathrm{M}+\mathrm{H}]^{+}$154.1226, found 154.1230.

(R)-5-methyl-3-(2-methylallyl)-2,3,4,7-tetrahydro-1Hazepin-3-ol (30). This compound was obtained from 22c $(0.65 \mathrm{~g})$ and purified by flash chromatography $\left(\mathrm{CHCl}_{3} /\right.$ $\mathrm{MeOH} 12: 1)$. Yield: $0.21 \mathrm{~g}, 60 \%$. Colorless oil. $[\alpha]_{\mathrm{D}}{ }^{25}=-$ $75.8\left(\mathrm{c}=0.8, \mathrm{CH}_{2} \mathrm{Cl}_{2}\right)$. ${ }^{\mathrm{H}} \mathrm{HMR}\left(300 \mathrm{MHz}, \mathrm{CDCl}_{3}\right.$, $333 \mathrm{~K}): \delta 1.75(\mathrm{~s}, 3 \mathrm{H}) ; 1.85(\mathrm{~s}, 3 \mathrm{H}) ; 2.2(\mathrm{~s}, 2 \mathrm{H}) ; 2.23(\mathrm{~d}, J$ $=15.1 \mathrm{~Hz}, 1 \mathrm{H}) ; 2.36(\mathrm{~d}, J=15.1 \mathrm{~Hz}, 1 \mathrm{H}) ; 2.67(\mathrm{~s}$ broad, $2 \mathrm{H}) ; 2.74(\mathrm{~d}, J=12.8 \mathrm{~Hz}, 1 \mathrm{H}) ; 2.98\left(\mathrm{~d}, J_{l}=12.8 \mathrm{~Hz}, 1 \mathrm{H}\right) ;$ $3.21(\mathrm{~m}, 1 \mathrm{H}) ; 3.32\left(\mathrm{dd}, J_{1}=15.4 \mathrm{~Hz}, J_{2}=7.0 \mathrm{~Hz}, 1 \mathrm{H}\right) ; 4.72$ $(\mathrm{d}, 1 \mathrm{H}, J=0.8 \mathrm{~Hz}) ; 4.87(\mathrm{~s}, 1 \mathrm{H}) ; 5.65(\mathrm{~m}, 1 \mathrm{H}) .{ }^{13} \mathrm{C} \mathrm{NMR}$ $\left(75 \mathrm{MHz}, \mathrm{CDCl}_{3}, 333 \mathrm{~K}\right): \delta 24.5\left(\mathrm{CH}_{3}\right) ; 27.5\left(\mathrm{CH}_{3}\right) ; 45.7$ $\left(\mathrm{CH}_{2}\right) ; 47.9\left(\mathrm{CH}_{2}\right) ; 48.5\left(\mathrm{CH}_{2}\right) ; 61.7\left(\mathrm{CH}_{2}\right) ; 69.7(\mathrm{C}) ; 114.7$ $\left(\mathrm{CH}_{2}\right) ; 124.8(\mathrm{CH}) ; 136.7(\mathrm{C}) ; 142.6(\mathrm{C})$. IR (film): 3330 (broad), 3070, 1645, $890 \mathrm{~cm}^{-1}$. HRMS calcd for $\mathrm{C}_{11} \mathrm{H}_{20} \mathrm{NO}$ $[\mathrm{M}+\mathrm{H}]^{+} 182.1539$, found 182.1543 .

(R)-3-methoxy-5-methyl-3-(2-methylallyl)-2,3,4,7tetrahydro-1H-azepine (31). This compound was obtained from 24c $(0.85 \mathrm{~g})$ and purified by flash chromatography $\left(\mathrm{CHCl}_{3} / \mathrm{MeOH} 15: 1\right)$. Yield: $0.28 \mathrm{~g}, 59 \%$. Colorless oil. $[\alpha]_{\mathrm{D}}{ }^{25}=+19.8\left(\mathrm{c}=1.0, \mathrm{CH}_{2} \mathrm{Cl}_{2}\right)$. ${ }^{\mathrm{H}} \mathrm{H}$ NMR $\left(400 \mathrm{MHz}, \mathrm{CDCl}_{3}\right): \delta 1.73(\mathrm{~s}, 3 \mathrm{H}) ; 1.75(\mathrm{~s}, 3 \mathrm{H}) ; 2.8(1 \mathrm{H})$; $2.14(\mathrm{~d}, J=14.4 \mathrm{H}<, 1 \mathrm{H}) ; 2.21(\mathrm{~d}, J=14.4 \mathrm{~Hz}, 1 \mathrm{H}) ; 2.27$ $(\mathrm{d}, J=15.2 \mathrm{~Hz}, 1 \mathrm{H}) ; 2.40(\mathrm{~d}, J=15.2 \mathrm{~Hz}, 1 \mathrm{H}) ; 2.78(\mathrm{~d}, J=$ $14.5 \mathrm{~Hz}, 1 \mathrm{H}) ; 3.04(\mathrm{~d}, J=15.5 \mathrm{~Hz}, 1 \mathrm{H}) ; 3.17(\mathrm{~m}, 1 \mathrm{H}) ; 3.18$ $(\mathrm{s}, 3 \mathrm{H}) ; 3.32\left(\mathrm{dd}, J_{1}=16.1 \mathrm{~Hz}, J_{2}=5.4 \mathrm{~Hz}, 1 \mathrm{H}\right), 4.70(\mathrm{~s}$, $1 \mathrm{H}) ; 4.81(\mathrm{~s}, 1 \mathrm{H}) ; 5.49(\mathrm{~m}, 1 \mathrm{H}) \cdot{ }^{13} \mathrm{C} \mathrm{NMR}(100 \mathrm{MHz}$, $\left.\mathrm{CDCl}_{3}\right): \delta 24.0\left(\mathrm{CH}_{3}\right) ; 27.0\left(\mathrm{CH}_{3}\right) ; 41.0\left(\mathrm{CH}_{2}\right) ; 42.3\left(\mathrm{CH}_{2}\right)$; $48.3\left(\mathrm{CH}_{2}\right) ; 49.2\left(\mathrm{CH}_{3}\right) ; 58.3\left(\mathrm{CH}_{2}\right) ; 77.0(\mathrm{C}) ; 114.5\left(\mathrm{CH}_{2}\right)$; $126.3(\mathrm{CH}) ; 134.8$ (C); 142.0 (C). IR (film): 3380 (broad), $3075,1645,1460,890 \mathrm{~cm}^{-1}$. HRMS calcd for $\mathrm{C}_{12} \mathrm{H}_{22} \mathrm{NO}$ $[\mathrm{M}+\mathrm{H}]^{+}$196.1696, found 196.1692.
(R, Z)-3-methoxy-5-methyl-3-(2-methylallyl)-1,2,3,4,7,8hexahydroazocine (32). This compound was obtained from $24 \mathrm{e}(0.75 \mathrm{~g})$ and purified by flash chromatography $\left(\mathrm{CHCl}_{3} / \mathrm{MeOH}\right.$ 20:1). Yield: $0.22 \mathrm{~g}, 52 \%$. Colorless oil. $[\alpha]_{\mathrm{D}}{ }^{25}=-69.9\left(\mathrm{c}=1.5, \mathrm{CH}_{2} \mathrm{Cl}_{2}\right) .{ }^{\mathrm{P}} \mathrm{H} \mathrm{NMR}(300 \mathrm{MHz}$ $\left.\mathrm{CDCl}_{3}, 300 \mathrm{~K}\right): \delta(\mathrm{s}, 6 \mathrm{H}) ; 1.93-2.07(\mathrm{~m}, 2 \mathrm{H}) ; 2.15(\mathrm{~d}, J=$ $14.8 \mathrm{~Hz}, 1 \mathrm{H}) ; 2.17(\mathrm{~m}, 1 \mathrm{H}) ; 2.21(\mathrm{~d}, J=13.4 \mathrm{~Hz}, 1 \mathrm{H}) ; 2.27$ $(\mathrm{d}, J=13.4 \mathrm{~Hz}, 1 \mathrm{H}) ; 2.28(\mathrm{~d}, J=14.8 \mathrm{~Hz}, 1 \mathrm{~Hz}) ; 2.59, J=$ $14.4 \mathrm{~Hz}, 1 \mathrm{H}) ; 2.61(\mathrm{~m}, \mathrm{H}) ; 3.22(\mathrm{~s}, 3 \mathrm{H}) ; 4.76(\mathrm{~d}, J=0.8 \mathrm{~Hz}$ $1 \mathrm{H}) ; 4.83(\mathrm{~d}, J=0.8 \mathrm{~Hz}, 1 \mathrm{H}) ; 5.41(\mathrm{~m}, 1 \mathrm{H}) .{ }^{13} \mathrm{C}$ NMR $(75$ $\left.\mathrm{MHz}, \mathrm{CDCl}_{3}, 300 \mathrm{~K}\right): \delta 24.0\left(\mathrm{CH}_{3}\right) ; 26.3\left(\mathrm{CH}_{3}\right) ; 29.6$ $\left(\mathrm{CH}_{2}\right) ; 38.3\left(\mathrm{CH}_{2}\right) ; 41.6\left(\mathrm{CH}_{2}\right) ; 48.9\left(\mathrm{CH}_{3}\right) ; 49.2\left(\mathrm{CH}_{2}\right)$ $53.3\left(\mathrm{CH}_{2}\right) ; 82.1(\mathrm{C}) ; 114.2\left(\mathrm{CH}_{2}\right) ; 123.8(\mathrm{CH}) ; 137.7(\mathrm{C})$; 142.3 (C). IR (film): 3370 (ancha); $3075,1640,690 \mathrm{~cm}^{-1}$ HRMS calcd for $\mathrm{C}_{13} \mathrm{H}_{24} \mathrm{NO}[\mathrm{M}+\mathrm{H}]^{+} 210.1852$, found 210.1849 .

(S)-3-methoxy-3-vinyl-1,2,3,6-tetrahydropyridine (33). This compound was obtained from 25a $(0.75 \mathrm{~g})$ and purified by flash chromatography $\left(\mathrm{CHCl}_{3} / \mathrm{MeOH} 15: 1\right)$ Yield: $0.20 \mathrm{~g}, 56 \%$. Colorless oil. $[\alpha]_{\mathrm{D}}^{25}=+52.1(\mathrm{c}=0.5$, $\left.\mathrm{CH}_{2} \mathrm{Cl}_{2}\right) .{ }^{1} \mathrm{H} \mathrm{NMR}\left(300 \mathrm{MHz}, \mathrm{CDCl}_{3}\right): \delta 2.30$ (broad, $1 \mathrm{H}$ ); $2.69(\mathrm{~d}, J=13.2 \mathrm{~Hz}, 1 \mathrm{H}) ; 3.01(\mathrm{~d}, J=13.2 \mathrm{~Hz}, 1 \mathrm{H}) ; 3.21-$ $3.38(\mathrm{~m}, 2 \mathrm{H}) ; 3.24(\mathrm{~s}, 3 \mathrm{H}) ; 5.21(\mathrm{~d}, J=17.6 \mathrm{~Hz}, 1 \mathrm{H}), 5.27$ $(\mathrm{d}, J=10.8 \mathrm{~Hz}, 1 \mathrm{H}) ; 5.80\left(\mathrm{dd}, J_{1}=17.6 \mathrm{~Hz}, J_{2}=10.8 \mathrm{~Hz}\right.$, $1 \mathrm{H}) ; 5.99(\mathrm{~m} \mathrm{1H}) ,6.06(\mathrm{~m}, 1 \mathrm{H}) .{ }^{13} \mathrm{C} \mathrm{NMR}(75 \mathrm{MHz}$, $\left.\mathrm{CDCl}_{3}\right): \delta 44.9\left(\mathrm{CH}_{2}\right) ; 50.7\left(\mathrm{CH}_{3}\right) ; 52.7\left(\mathrm{CH}_{2}\right) ; 72.6(\mathrm{C})$; $116.9\left(\mathrm{CH}_{2}\right) ; 126.3(\mathrm{CH}) ; 132.4(\mathrm{CH}) ; 140.4(\mathrm{CH})$. IR (film): 3320 (broad), 3085, 1635, 1460, 720, $685 \mathrm{~cm}^{-1}$ HRMS calcd for $\mathrm{C}_{8} \mathrm{H}_{14} \mathrm{NO}[\mathrm{M}+\mathrm{H}]^{+} 140.1070$, found 140.1071

\section{(S)-3-methoxy-3-vinyl-2,3,6,7-tetrahydro-1H-azepine}

(34). This compound was obtained from 25d $(0.65 \mathrm{~g})$ and purified by flash chromatography $\left(\mathrm{CHCl}_{3} / \mathrm{MeOH}\right.$ 15:1). Yield: $0.22 \mathrm{~g}, 68 \%$. Colorless oil. $[\alpha]_{\mathrm{D}}{ }^{25}=-4.2(\mathrm{c}=1.2$ $\left.\mathrm{CH}_{2} \mathrm{Cl}_{2}\right) .{ }^{\mathrm{H}} \mathrm{NMR}\left(300 \mathrm{MHz}, \mathrm{CDCl}_{3}\right): \delta 2.28-2.35(\mathrm{~m}$, $2 \mathrm{H}) ; 2.89-3.20(\mathrm{~m}, 4 \mathrm{H}) ; 3.24(\mathrm{~s}, 3 \mathrm{H}) ; 3.50$ (broad, $1 \mathrm{H})$; 5.27-5.36 (m, 2H); $5.70(\mathrm{~m}, 2 \mathrm{H}) ; 6.05(\mathrm{~m}, 1 \mathrm{H}) .{ }^{13} \mathrm{C} \mathrm{NMR}$ $\left(75 \mathrm{MHz}, \mathrm{CDCl}_{3}\right): \delta 30.7\left(\mathrm{CH}_{2}\right) ; 47.8\left(\mathrm{CH}_{2}\right) ; 50.9\left(\mathrm{CH}_{3}\right)$ $55.9\left(\mathrm{CH}_{2}\right) ; 77.00(\mathrm{C}) ; 117.6\left(\mathrm{CH}_{2}\right) ; 131.7(\mathrm{CH}) ; 133.1$ $(\mathrm{CH}) ; 140.2$ (CH). IR (film): 3410 (broad); 3085, 1640 $146,755 \mathrm{~cm}^{-1}$. HRMS calcd for $\mathrm{C}_{9} \mathrm{H}_{16} \mathrm{NO}[\mathrm{M}+\mathrm{H}]^{+}$ 154.1226, found 154.1228 .

\section{Acknowledgements}

We acknowledge financial support provided by the Spanish MINECO (Project FEDER-CTQ2014-59870-P) and Junta de Castilla y León (Project FEDER-VA115P17).

\section{References}

[1] a) A. F. Pozharskii, A. T. Soldatenkov, Katritzky, A. R. Katritzky in Heterocycles in Life and Society: An Introduction to Heterocyclic Chemistry, Biochemistry and Applications, 2nd ed., Wiley-VCH, Weinheim, 2011; b) R. D. Taylor, M. MacCoss, A. D. G. Lawson, J. Med. Chem. 2014, 57, 5845-5859.

[2] For some reviews on ring-closing metathesis, see: a) R. H. Grubbs, S. Chang, Tetrahedron 1998, 54, 44134450; b) S. K. Armstrong, J. Chem. Soc., Perkin Trans I, 1998, 371-3788; c) A. Fürstner, Angew. Chem. Int. Ed. 2000, 39, 3012-3043; d) R. R. Schrock, A. H. Hoveyda, Angew. Chem. Int. Ed. 2003, 42, 4592-4633; e) A. H. Hoveyda, D. G. Gillingham, J. J. Van Veldhuizen, O. Kataoko, S. B. Garber, J. S. Kingsburg, J. P. A. Harrity, Org. Biomol Chem. 2004, 2, 8-23; f) K. C. Nicolaou, P. G. Bulger, D. Sarlah, Angew. Chem. Int Ed. 2005, 44, 4490-4525; g) S-Y. Han, S. Chang, in 
Handbook of Metathesis, ed. R. H. Grubbs, WileyVCH, Weinheim, 2008, Vol 2 pp 5-127.

[3] For some reviews on synthesis of oxygen containing heterocycles by ring-closing metathesis, see: a) A. Deiters, S. F. Martin, Chem. Rev. 2004, 104, 21992238; b) J. S. Clark, Chem. Commun. 2006, 35713581; c) J. D. Rainer, in Metathesis in Natural Product Synthesis: Strategies, Substrates and Catalysts. ed. J. Cossy, S. Arseniyadis, C. Meyer, Wiley-VCH, 2010, pp 87-127; d) R. Jacques, R. Pal, N. A. Parker, C. E. Sear, P. W. Smith, A. Ribaucort, D. M. Hodgson, Org. Biomol. Chem. 2016, 14, 5875-5893; e) D. M. Lindsay, in Synthesis of Heterocycles by Metathesis Reactions, ed. J. Prunet, Springer, Switzerland, 2017, pp 33-55.

[4] For some reviews on synthesis of nitrogen containing heterocycles by ring-closing metathesis, see: a) A. J. Phillips, A. D. Abell, Aldrichimica Acta, 1999, 32, 7589; b) S. A. M. W. van der Broek, S. A. Meeuwissen, F. L. van Delft, F. P. J. T. Rutjes, in Metathesis in Natural Product Synthesis: Strategies, Substrates and Catalysts, ed. J. Cossy, S. Arseniyadis, C. Meyer, Wiley-VCH, 2010, pp 45-85; c) P. Compain, D. Hazelard, in Synthesis of Heterocycles by Metathesis Reactions, ed. J. Prunet, Springer, Switzerland, 2017, pp 11-154. See also reference $2 \mathrm{a}$.

[5] For selected reviews on enantioselective olefin metathesis, see: a) A. H. Hoveyda, R. R. Schrock, Chem. Eur. J. 2001, 7, 945-950; b) A. H. Hoveyda, in Handbook of Metathesis, ed. R. H. Grubbs, WileyVCH, Weinheim, 2008, Vol 2 pp 128-150; c) A. H. Hoveyda, S. J. Malcolmson, S. J. Meek, A. R. Zhugralin, Ang. Chem., Int. Ed., 2010, 49, 34-44; d) A. H. Hoveyda, S. J. Malcolmson, S. J. Meek, A. R. Zhugranlin, in Metathesis In Natural Product Synthesis: Strategies, Substrates and Catalysts, ed. J. Cossy, S. Arseniyadis, C. Meyer; Wiley-VCH, Weinheim, 2010, pp 343-248; e) S. Kress, S. Blechert, Chem. Soc. Rev., 2012, 41, 4389-4408; f) A. H. Hoveyda, J. Org. Chem., 2014, 79, 4763-4792; g) B. Stenne, S. K. Collins, in Olefin Metathesis: Theory and Practice, ed. K. Grela, Wiley-VCH Weinheim, 2014; pp 233-267.

[6] a) D. S. La, J. B. Alexander, D. R. Cefalo, D. D. Graf, A. H. Hoveyda, R. R. Schrock, J. Am. Chem. Soc., 1998, 120, 9720-9721; b) S. J. Dolman, E. S. Sattely, A. H. Hoveyda, R. R. Schrock, J. Am. Chem. Soc., 2002, 124, 6991-6997; c) J. Hartung, P. K. Dornan, R. H. Grubbs. J. Am. Chem. Soc., 2014, 136, 13029-13037.

[7] For selected examples on diastereoselective olefin metathesis reactions, see: a) C. M. Huwe, J. Velder, S. Blechert, Angew. Chem. Int. Ed. Engl., 1996, 35, 23762378; b) M. Lautens, G. Hughes, Angew. Chem. Int. Ed., 1999, 38, 129-131; c) H. Oguri, S. Sasaki, T. Oishi, M. Hirama, Tetrahedron Lett., 1999, 40, 5405-5408; d) B. Schmidt, M. Westhus, Tetrahedron, 2000, 56, 24212426; e) D. J Wallace, C. J Cowden, D. J. Kennedy, M. S. Ashwood, I. F. Cottrell, U.-H. Dolling, Tetrahedron Lett.; 2000, 41, 2027-2029; f) D. J. Wallace, J. M. Goodman, D. J. Kennedy, A. J. Davies, C. J. Cowden, M. S. Ashwood, I. F. Cottrell, U-H. Dolling, P. J.
Reider, Org. Lett., 2001, 3, 671-674; g) D. J. Wallace, P. G. Bulger, D. J. Kennedy, M. S. Ashwood, I. F. Cottrell, U-H. Dolling, Synlett, 2001, 357-360; h) K. C. Nicolaou, G. Vassilikogiannakis, T. Montagnon, Angew. Chem. Int. Ed., 2002, 41, 3276-3280; i) P. A. Evans, J. Cui, G. P. Buffone; Angew Chem. Int. Ed., 2003, 42, 1734-1737 j) R. A. J. Wybrow, A. S. Edwards, N. G. Stevenson, H. Adams, C. Johnstone, J. P. A. Harrity, Tetrahedron, 2004, 60, 8869-8880; k) D. J. Wallace, Tetrahedron Lett. 2005, 591-594; 1) K. S. Dunne, F. Bisaro, B. Odell, J-M. Paris, V. Gouverneur, J. Org. Chem., 2005, 70, 10803-10809; m) B. Cheng, J. D. Sunderhaus, S. F. Martin, Org. Lett., 2010, 12, 3622-3625; n) J. S. Harvey, G. T. Giuffredi, V. Gouverneur, Org. Lett., 2010, 12, 1236-1239; o) J. Li, D. Lee, Chem Sci, 2012, 3, 3296-3301.

[8] a) V. Böhrsch, S. Blechert, Chem. Commun., 2006, 1968-1970; b) Y Onodera, K. Hirota, Y. Suga, K. Konoki, M. Yotsu-Yamashita, M. Sasaki, H. Fuwa. J. Org. Chem. 2016, 81, 8234-8252.

[9] a) K. S. Feldman, J. F. Antoline, Tetrahedron, 2013, 69, 1434-1445; b) A. Umehara, H. Ueda, H. Tokuyama, Org. Lett., 2014, 16, 2526-2529.

[10] a) Y. Fukuda, M. Shindo, K. Shishido, Org. Lett., 2003, 5, 749-751; b) Y. Murakami, M. Shindo, K. Shishido, Synlett, 2005, 664-666.

[11] a) J. Nieto, C. Andrés, A. Perez-Encabo, Org. Biomol. Chem. 2015,13, 9118-9126; b) C. Andrés, I. González, J. Nieto, C. D. Rosón, Tetrahedron, 2009, 659728 9736; c) R. Pedrosa, C. Andrés, J. Nieto, C. PérezCuadrado, I. San Francisco, Eur. J. Org. Chem., 2006, 3259-3265; d) R. Pedrosa, C. Andrés, L. Martín, J. Nieto, C. Rosón, J. Org. Chem. 2005, 70, 4332-4337; e) R. Pedrosa, C. Andrés, J. P. Duque-Soladana, A. Maestro, J. Nieto, Tetrahedron: Asymmetry, 2003, 14, 2985-2990.

[12] In a previous work we have described the synthesis of chiral azepin-3-ol and azocin-3-ol derivatives by a sequential diastereoselective addition of unsaturated Grignard reagents to 2-acyl-perhydro-1,3-benzoxazines and RCM but the new stereocenter is not created during the RCM reaction. R. Pedrosa, C. Andrés, A. GutiérrezLoriente, J. Nieto, Eur. J. Org. Chem., 2005, 24492458.

[13] X-C. He, E. L. Eliel, Tetrahedron, 1987, 43, 49794987.

[14] E. L. Eliel, X-C. He, J. Org. Chem., 1990, 55, $2114-$ 2119.

[15] For other examples of catalyst dependant diastereoselectivity see: a) C. M. Huwe, J. Velder, S. Blechert, Angew. Chem. Int. Ed. Engl., 1996, 35, 23762378; b) M. Lautens, G. Hughes, Angew. Chem. Int. Ed., 1999, 38, 129- 131; c) R. A. J. Wybrow, N. G. Stevenson, J. P. A. Harrity, Synlett, 2004, 140-142; d) D. J. Wallace, Tetrahedron Lett., 2005, 46, 591-594; e) Y. Onodera, K. Hirota, Y. Suga, K. Konoki, M. YotsuYamashita, M. Sasaki, H. Fuwa, J Org. Chem.; 2016, 81, 8234-8252. 
[16] CCDC 1872085 (7c), CCDC 1872081 (8d), CCDC 1872084 (8e), CCDC 1872078 (8g), CCDC 1872086 (9), CCDC 1872079 (25a), CCDC 1872080 (24c), CCDC 1872083 (27d) and CCDC 1872082 (28) contains the supplementary crystallographic data for this paper. These data can be obtained free of charge from The Cambridge Crystallographic Data Centre via www.ccdc.cam.ac.uk/data_request/cif.

[17] C. Andrés, J. Nieto, R. Pedrosa and M. Vicente, J. Org. Chem., 1998, 63, 8570-8573.

[18] a) B. Schmidt, H. Wildemann, J. Org. Chem., 2000, 65, 5817-5822; b) Y. Fukuda, H. Sasaki, M. Shindo, K. Shishido, Tetrahedron Lett., 2002, 43, 2047-2049; (d) J. Li, D. Lee, Chem. Sci., 2012, 3, 3296-3301. See also references $10 \mathrm{a}$ and $10 \mathrm{~b}$.

[19] Despite the fact that the Grubbs' ruthenium carbene 5 show remarkable functional group tolerance, the tolerance is generally poor for amines: a) G. C. Fu, S.B. T. Nguyen, R. H. Grubbs, J. Am. Chem. Soc. 1993 , 115, 9856-9857; b) Y.-S. Shon, T. R. Lee, Tetrahedron Lett. 1997, 38, 1283-1286; c) A. Briot, M. Bujard, V. Gouverneur, S. P. Nolan, C. Mioskowski, Org. Lett. 2000, 2, 1517-1519.

[20] It has been demonstrated that ammonium salts are tolerated by the Grubbs' ruthenium catalyst: a) D. L. Wright, J. P. Schulte, II, M. A. Page, Org. Lett. 2000, 2, 1847-1850; b) K. Shimizu, M. Takimoto, M. Mori, Org. Lett. 2003, 5, 2323-2325. See also references 12 and $18 \mathrm{a}$.

[21] E. J. Corey, A. Venkateswarlu, J. Am. Chem. Soc. 1972, 94, 6190-6191.

[22] R. Pedrosa, C. Andrés, J. Nieto, S. del Pozo, J. Org. Chem., 2003, 68, 4923-4931. 
